\title{
Past Results, Present Trends, and Future Challenges in Intrabody Communication
}

\author{
David Naranjo-Hernández $\mathbb{D}^{1},{ }^{1}$ Amparo Callejón-Leblic $\left(\mathbb{D},{ }^{2}\right.$ Željka Lučev Vasić $\left(\mathbb{D},{ }^{3}\right.$ \\ MirHojjat Seyedi $\oplus^{1},{ }^{4}$ and Yue-Ming Gao $\oplus^{5}$ \\ ${ }^{1}$ Biomedical Research Networking Centre in Bioengineering, Biomaterials and Nanomedicine (CIBER-BBN), Seville, Spain \\ ${ }^{2}$ Biomedical Engineering Group, University of Seville, Seville, Spain \\ ${ }^{3}$ Faculty of Electrical Engineering and Computing, University of Zagreb, Zagreb, Croatia \\ ${ }^{4}$ Department of Electrical Engineering, Islamic Azad University, Urmia Branch, Urmia, Iran \\ ${ }^{5}$ Fuzhou University, Fuzhou, China
}

Correspondence should be addressed to David Naranjo-Hernández; dnaranjo@us.es

Received 18 August 2017; Accepted 25 December 2017; Published 11 March 2018

Academic Editor: Pierre-Martin Tardif

Copyright (c) 2018 David Naranjo-Hernández et al. This is an open access article distributed under the Creative Commons Attribution License, which permits unrestricted use, distribution, and reproduction in any medium, provided the original work is properly cited.

Intrabody communication (IBC) is a wireless communication technology using the human body to develop body area networks (BANs) for remote and ubiquitous monitoring. IBC uses living tissues as a transmission medium, achieving power-saving and miniaturized transceivers, making communications more robust against external interference and attacks on the privacy of transmitted data. Due to these advantages, IBC has been included as a third physical layer in the IEEE 802.15.6 standard for wireless body area networks (WBANs) designated as Human Body Communication (HBC). Further research is needed to compare both methods depending on the characteristics of IBC application. Challenges remain for an optimal deployment of IBC technology, such as the effect of long-term use in the human body, communication optimization through more realistic models, the influence of both anthropometric characteristics and the subject's movement on the transmission performance, standardization of communications, and development of small-size and energy-efficient prototypes with increased data rate. The purpose of this work is to provide an indepth overview of recent advances and future challenges in human body/intrabody communication for wireless communications and mobile computing.

\section{Introduction}

Intrabody communication (IBC) techniques make use of the human body as a transmission medium for electrical signals to interconnect devices in wireless body area networks (WBANs). These devices can be both on-body and implanted (in-body) and communicate with each other and with a central device through low power consumption and low data rate body channels [1]. These links connect two on-body devices, two in-body devices, and an on-body with an in-body device [2], as described in Figure 1. These devices are both sensors that record and process physiological variables of interest and actuators that receive information and generate some type of response, for example, electronic drug delivering pumps. The central device, which coordinates and manages the operation of the network, sends data through an external long-distance link to a medical decision center for evaluation. This link is usually implemented with Wireless Local Area Network (WLAN) standards, which support larger amounts of data [3]. This way, this central device acting as an external gateway supports the higher transmission power in the network, thus minimizing the power consumption of the rest of the sensors and actuators [4].

The IEEE 802.15.6 standard ratified in 2012 proposes three different physical layers (PHY) for the development of new WBAN, including intrabody communication technique, designated as Human Body Communication (HBC). Based on Electrostatic Field Communication (EFC) introduced by 


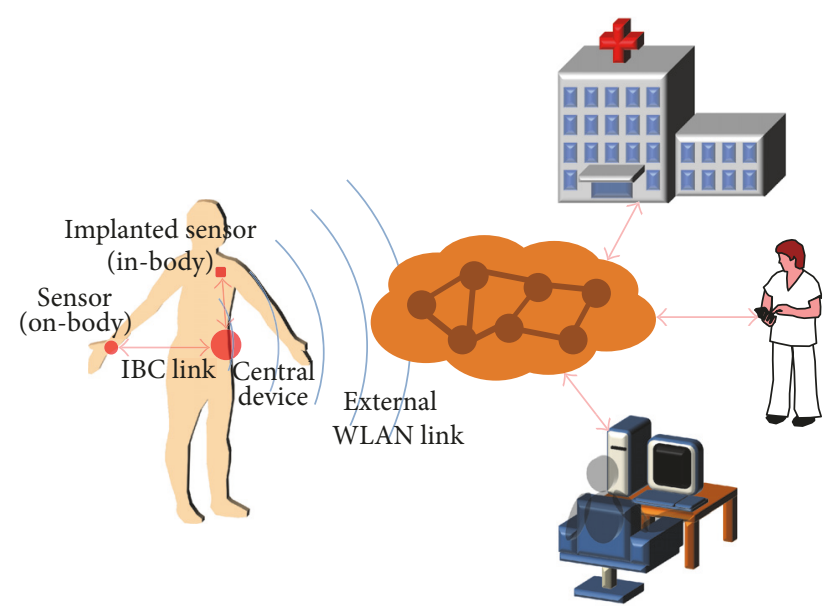

FIgURE 1: Body area network scheme with IBC links: in-body and on-body sensors exchange data between each other and with a central device through channels formed through the human body. The data are then sent to a medical decision center via an external WLAN link, where they are processed and stored.

Zimmerman in 1996 [5], this standard defines a central frequency of $21 \mathrm{MHz}$ with data rates in the range from 164 to $1312.5 \mathrm{~kb} / \mathrm{s}[6]$. In the IBC architecture originally proposed by Zimmerman, a transmitter placed on the skin modulates an electric field, which is driven by small currents through the human body towards the receiver, which demodulates the signal by obtaining the encoded information [7]. The environment provides a signal return path towards the transmitter by electrostatic close-field coupling. In general terms, the IBC communication defined by Zimmerman is based on a transmitter that generates an electric potential, thus disturbing its immediate external environment and a receiver that detects such disturbance. The carrier frequencies used are sufficiently low to be associated with near-field components in the range of distances covered through the human body, thus minimizing energy consumption and interferences with nearby devices. Also, voltage levels are well below the maximum thresholds allowed to guarantee safety. In the near-field coupling, the power decreases with the cube of the distance; however, in the BAN scenario, this represents a double advantage. On the one hand, the distances to be covered in the human body are small enough to guarantee signal reception, and, on the other hand, the signal is not radiated out to the environment, therefore improving the energy efficiency and the robustness of the system.

Generally, IBC communication is established through the human body; nevertheless the signal paths may vary depending on the configuration of the electrodes and the different signal coupling modes. In the literature, in addition to the electrostatic or capacitive coupling technique, the galvanic coupling technique has also been proposed. The first establishes two signal paths, a first path through the human body and a second return path through the air by electrostatic coupling. This feature allows the interconnection of devices that are both deployed on the same body surface or close to it, without the need for direct contact with the skin.
Alternatively, the galvanic coupling technique establishes a single signal differential path through a current flow that penetrates into internal tissues. Due to this characteristic, galvanic coupling has frequently been proposed as a viable alternative for the communication between implanted sensors.

The purpose of this paper is to provide a complete overview of recent advances and future challenges in human body/intrabody communication for wireless communications and mobile computing. The content of the survey is organized in the following sections and subsections:

(i) Section 2: IBC physics and modeling

(1) Section 2.1: capacitive and galvanic coupling;

(2) Section 2.2: bioelectric basis of signal transmission through human tissues;

(3) Section 2.3: safety requirements and regulations in IBC;

(4) Section 2.4: modeling of IBC channels.

(ii) Section 3: measurement issues

(1) Section 3.1: electrodes and body coupling issues;

(2) Section 3.2: measurement issues and experimental characterization;

(3) Section 3.3: influence of anthropometric characteristics and position issues;

(4) Section 3.4: sources of artifacts and noise in IBC.

(iii) Section 4: IBC transceivers and devices

(1) Section 4.1: design and implementation of IBC transceivers;

(2) Section 4.2: IBC modulation and power consumption.

(iv) Section 5: IBC applications and systems

(1) Section 5.1: IBC in medicine, systems, and applications;

(2) Section 5.2: IBC for implanted systems;

(3) Section 5.3: nanoscale intrabody communication systems.

(v) Section 6: future challenges of IBC.

\section{IBC Physics and Modeling}

In this section an overview of underlying physics, bioelectric mechanisms, and channel models for IBC is presented. First, the main two coupling techniques proposed in the literature, galvanic and capacitive coupling, are reviewed, compared, and discussed. Then, the bioelectric principles of signal transmission through human tissues, which allow the IBC phenomenon to be explained, are described in this section. Finally, the major safety requirements in IBC, as well as the main channel modeling approaches, are shown. 


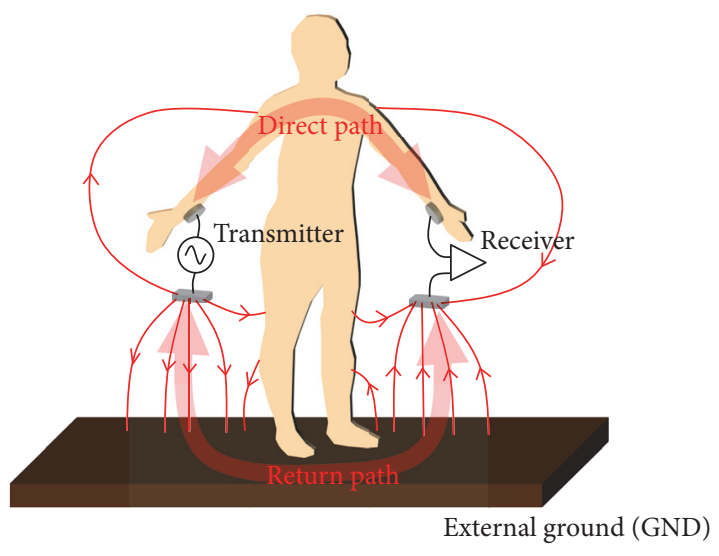

FIgURE 2: Schematic of the capacitive coupling technique. The principle of operation of this communication scheme is based on near-field electrostatic coupling, in such a way that the signal is confined by surface waves on the direct path formed through the human body.

2.1. Capacitive and Galvanic Coupling. In this subsection, a detailed explanation of the characteristics of the two previously mentioned techniques, capacitive and galvanic coupling, is provided.

2.1.1. Capacitive Coupling. In the capacitive coupling technique, an electrical potential is generated between the signal and ground electrodes of the transmitter device (TX). This potential is sensed by a receiver device (RX) which also has a pair of signal and ground electrodes. While the signal electrode can be placed directly on the surface of the human body or very close to it, a floating ground electrode is directed towards the external environment. Figure 2 shows a schematic of the distribution of electric fields in the capacitive coupling technique. The electric field induced by the TX signal electrode is coupled to the human body and passes through it establishing a first direct signal path. Part of this field is lost through the feet towards the external ground. In turn, due to the fact that the grounds of the TX and the RX are not physically connected, a second return path through the air appears. In this way, the electric field received at the RX is very weak but sufficient enough to be detected [17]. Finally, the transmission loop is closed through the return path through the air between the TX and RX grounds. Although the human body behaves like a conductor, the couplings between the electrodes, air, and external ground can be modeled as capacitances, hence the name of this technique [18]. The values of these capacitances are very small, often making the signals obtained at the receiving end very weak due to the poor return paths being established through the external ground [19]. The main advantage of this technique compared with other radio frequency techniques is that much of the signal is confined to the body surface as the human body behaves like an electric conductor, thus minimizing the transmission power required.

In summary, the prevalent signal channel is the capacitive return path established through the air, which makes it

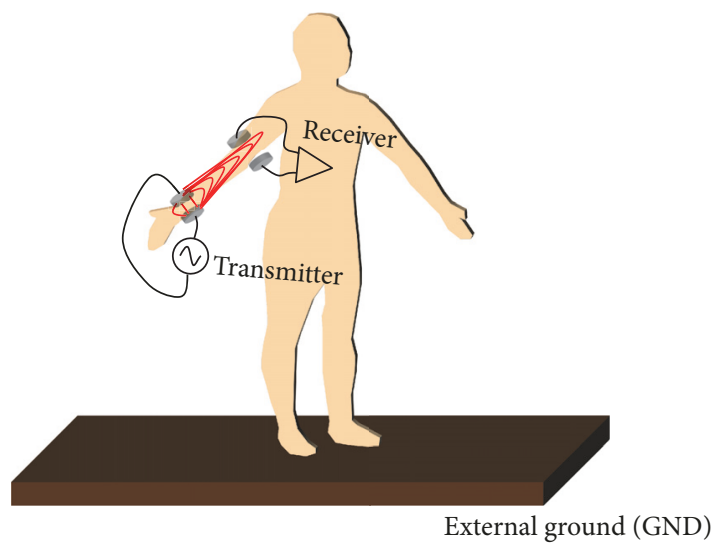

FIGURE 3: Schematic of the galvanic coupling technique. This technique is based on a differential configuration scheme in which an electric current is applied into the skin, thus leading to two different current pathways: a primary current flow through the TX electrodes and a secondary flow through the inner tissues towards the RX electrodes.

strongly dependent on the external environment conditions [20]. In addition, as the frequency increases, the signal radiation into the air is no longer negligible. Some of these disadvantages are minimized with the use of the galvanic coupling mode, which is presented in the following section.

2.1.2. Galvanic Coupling. In the galvanic coupling technique, four electrodes are attached to the human body, two at the TX site for the injection of an electric current and two at the RX end. Figure 3 shows a schematic of the distribution of electric currents in the coupling technique. Since electric current is injected into the human body, galvanic IBC devices must comply strictly with electromagnetic compatibility regulations, avoiding interference with other biomedical devices. In addition, in order to avoid any adverse health problem, transmitted signals must be well below the limits specified by international guidelines institutions [21-23]. Transmission frequencies are selected from $10 \mathrm{kHz}$ to not interfere with other endogenous physiological signals. In addition, the typical values of signal amplitude are in the range of 1 to $2 \mathrm{~mA}$ [24]. The experimental results demonstrate that the galvanic coupling technique is an appropriate method for the transmission of signals between devices placed on the chest, or at short distances on the limbs, in the range between $10 \mathrm{kHz}$ and $1 \mathrm{MHz}$ [25]. In addition, since the injected currents are very weak, this mode requires a lower transmitted signal level than those other forms of communication based on radio frequency standards.

2.1.3. Comparison and Discussion. Each of these techniques presents its own advantages and disadvantages, being more or less viable depending on the application, particular design requirements, frequency range, and distances to be covered in the human body. Capacitive coupling has been extensively used in the literature, thus leading to transceivers with data rates of up to $10 \mathrm{Mbps}$ [26]. However, since this 
method requires an external ground reference, the viability of the communication sometimes depends on the influence of environmental conditions, isolation strategies used, and so forth, being very sensitive to interferences and subject movements [27, 28]. On the other hand, galvanic coupling has proved to be more robust against interference, since the signal is differentially applied through two electrodes without a common reference [29]. Since the signal is mainly confined through the body channel, the transmission will depend to a greater extent on the frequency-dependent dielectric properties of tissues [30]. In contrast, galvanic coupling presents the limitation of a reduced bandwidth and a lower data rate. Table 1 presents a brief comparison of the main characteristics of both techniques.

\subsection{Bioelectric Basis of Signal Transmission through Human}

Tissues. The problem of the characterization of the human body as a communication channel has been addressed in the literature from a theoretical perspective, with the proposal of different models to analyze the main mechanisms of IBC transmission, and, from an experimental viewpoint, with the use of electronic setups specifically designed for the measurement of human body attenuation. As highlighted in previous sections, IBC communication techniques are based on the transmission of electric signals through the human body as a transmission medium, which presents bioelectric properties that must necessarily be taken into account as the fundamental basis of the theoretical models and experimental setups developed. The study of the interaction of electric fields with the human body in IBC transmission implies the need for a consistent electrical characterization of the dielectric properties of biological tissues, that is, conductivity and permittivity, which, respectively, determine the ohmic currents and the magnitude of the polarization effects, and they are in turn frequency dependent. In living tissues, this dependency is intimately ligated to the interaction between the electromagnetic fields and the ions and molecules contained at both the intra- and extracellular mediums. The main dielectric properties of biological tissues were first reported by Foster and Schwan in 1989 [123], where it was shown that the dispersions observed in living tissues are fundamentally four (three main dispersions: $\alpha, \beta$, and $\gamma$ at low, medium, and high frequencies; and a minor dispersion, $\delta$, at very high frequencies). Each of these dispersive regions is the manifestation of a mechanism of polarization governed by a time constant $(\tau)$, which in first approximation offers the following expression for the complex electrical permittivity $\left(\epsilon_{r}^{*}\right)$ of a medium as a function of angular frequency $(\omega=$ $2 \pi f)$ :

$$
\epsilon_{r}^{*}=\epsilon_{r}^{\prime}(\omega)-j \epsilon_{r}^{\prime \prime}(\omega)=\epsilon_{\infty}+\frac{\epsilon_{s}-\epsilon_{\infty}}{1+j \omega \tau} .
$$

This is the well-known Debye equation, where $\epsilon_{r}^{\prime}(\omega)$ and $\epsilon_{r}^{\prime \prime}(\omega)$ are the real and imaginary part of the complex permittivity, $\epsilon_{\infty}$ is the permittivity at frequencies where $\omega \tau \gg$ $1, \epsilon_{s}$ is the permittivity at frequencies where $\omega \tau \ll 1$, and $j$ is the imaginary unit $\left(j^{2}=-1\right)$. However, the complexity of the structure and composition of biological materials is such that

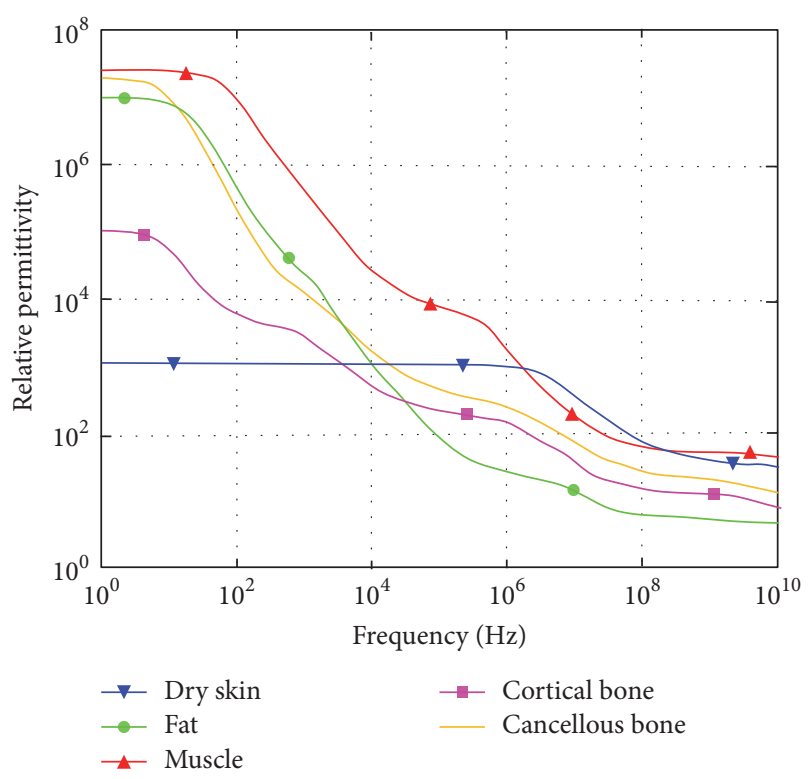

FIgURE 4: Relative permittivity $\epsilon_{r}$ for dry skin, fat, muscle, and cortical and cancellous bone reported by Gabriel et al. in [8].

Debye's expression fails to emulate their dielectric properties in a wide range of frequencies, and, therefore, distribution parameters $(0<\alpha<1)$ in each of the dispersive regions need to be introduced, giving rise to the famous Cole-Cole equation [124]:

$$
\epsilon_{r}^{*}=\epsilon_{\infty}+\sum_{n=1}^{4} \frac{\Delta \epsilon_{n}}{1+(j \omega \tau)^{1-\alpha_{n}}}+\frac{\sigma_{s}}{j \omega \epsilon_{0}},
$$

where $\sigma_{s}$ is the static conductivity of tissue and $\epsilon_{0}$ is the permittivity of free space. In addition, the value of the complex conductivity can be found according to

$$
\sigma^{*}(\omega)=j \omega \epsilon_{0} \epsilon^{*} .
$$

One of the most relevant works in this field was presented by Gabriel et al. in 1996 [8, 31], where the conductivity and permittivity of some of the most important living tissues were reported from $10 \mathrm{~Hz}$ to $20 \mathrm{GHz}$. In addition, Gabriel et al. also provided the corresponding Cole-Cole model parameters for these tissues. Table 2 shows the parameters reported for some of the human tissues more commonly used in the IBC literature (dry skin, fat, muscle, and bone). Figures 4 and 5 show the permittivity and conductivity values for these tissues as a function of frequency.

It must be noticed that $\beta$ dispersion is mainly due to the polarization of cell membranes, whose behavior can be modeled as a capacitance, which appears due to the existence of regions of high conductivity (extracellular and intracellular mediums) separated by regions of low conductivity (cell membranes). This way, at low frequencies, only a very small current will flow through the extracellular fluid surrounding the cells due to the high impedance of the cell membrane (see Figure 6(a)). As the frequency increases, the "membrane plates" are charged and discharged very rapidly, reducing 
TABLE 1: Main differences between galvanic and capacitive coupling techniques.

\begin{tabular}{|c|c|c|}
\hline & Galvanic coupling & Capacitive coupling \\
\hline Type of signal injected & Alternating current flow & Electrostatic potential \\
\hline Electrodes & $\begin{array}{l}\text { One pair of TX electrodes and another pair of RX } \\
\text { electrodes connected to the body }\end{array}$ & $\begin{array}{l}\text { In TX and RX, the signal electrodes are connected to the } \\
\text { human body, and the ground electrodes remain floating } \\
\text { towards the external air }\end{array}$ \\
\hline Contact & Direct contact with the human body is required & Direct contact with the human body is not required \\
\hline Signal coupling & Differential & It needs an external common ground \\
\hline Signal paths & The dominant signal path is the human body & $\begin{array}{l}\text { Two signal paths: a direct path through the human body } \\
\text { and a return path through the external ground }\end{array}$ \\
\hline Attenuation & Dependent on bioelectric properties of tissues & Dependent on external environmental conditions \\
\hline Data rate & Low data rates & Higher data rates \\
\hline Interference & Sensitive to body part and placement of electrodes & $\begin{array}{l}\text { Sensitive to other nearby devices that can be capacitively } \\
\text { coupled }\end{array}$ \\
\hline
\end{tabular}

TABLE 2: Parameters of the Cole-Cole model reported for Gabriel et al. in [31] for relevant IBC tissues.

\begin{tabular}{lccccccccccccccc}
\hline & $\epsilon_{\infty}$ & $\Delta \epsilon_{1}$ & $\tau_{1}(\mathrm{ps})$ & $\alpha_{1}$ & $\Delta \epsilon_{2}$ & $\tau_{2}(\mathrm{~ns})$ & $\alpha_{2}$ & $\Delta \epsilon_{3}$ & $\tau_{3}(\mathrm{~s})$ & $\alpha_{3}$ & $\Delta \epsilon_{4}$ & $\tau_{4}(\mathrm{~ms})$ & $\alpha_{4}$ & $\sigma$ \\
\hline Dry skin & 4.0 & 32.0 & 7.23 & 0.00 & 1100 & 32.48 & 0.20 & 0.0 & & & 0.0 & & 0.0002 \\
Fat & 2.5 & 3.0 & 7.96 & 0.20 & 15 & 15.92 & 0.10 & $3.3 \cdot 10^{4}$ & 159.15 & 0.05 & $1 \cdot 10^{7}$ & 7.958 & 0.01 & 0.0100 \\
Muscle & 4.0 & 50.0 & 7.23 & 0.10 & 7000 & 353.68 & 0.10 & $1.2 \cdot 10^{6}$ & 318.31 & 0.10 & $2.5 \cdot 10^{7}$ & 2.274 & 0.00 & 0.2000 \\
Cortical bone & 2.5 & 10.0 & 13.26 & 0.20 & 180 & 79.58 & 0.20 & $5 \cdot 10^{4}$ & 159.15 & 0.20 & $1 \cdot 10^{5}$ & 15.915 & 0.00 & 0.0200 \\
Cancellous bone & 2.5 & 18.0 & 13.26 & 0.22 & 300 & 79.58 & 0.25 & $2 \cdot 10^{4}$ & 159.15 & 0.20 & $2 \cdot 10^{7}$ & 15.915 & 0.00 & 0.0700 \\
\hline
\end{tabular}

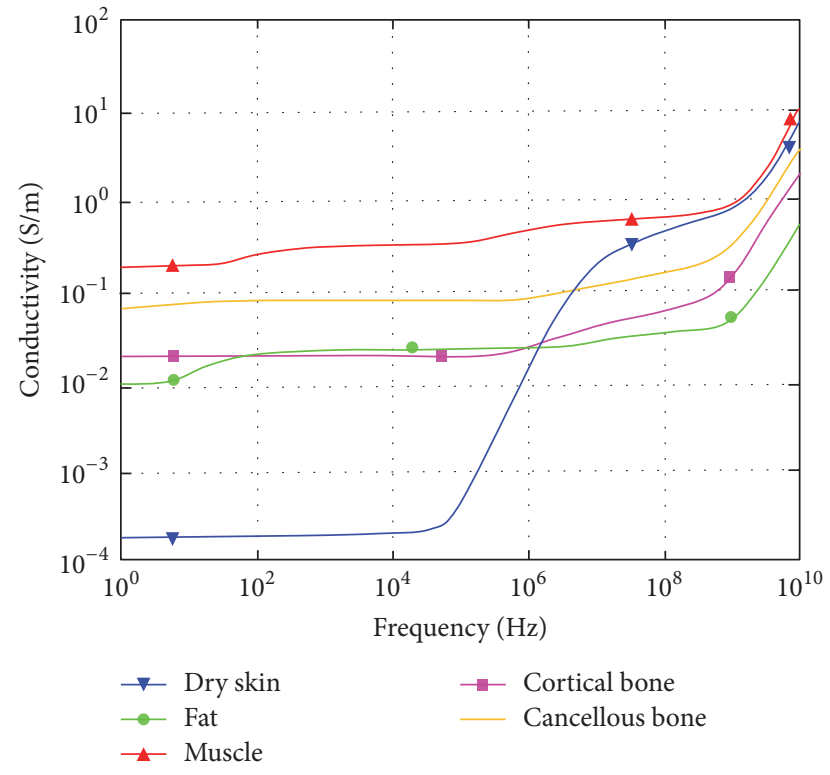

FIGURE 5: Electric conductivity $\sigma$ for dry skin, fat, muscle, and cortical and cancellous bone reported by Gabriel et al. in [8].

the membrane impedance and allowing a small amount of current to penetrate into the cell. This way, the current flows directly through the intra- and extracellular medium from tens of $\mathrm{MHz}$ (see Figure 6(b)). This effect leads to the increment of conductivity and the decrement of permittivity seen in Figures 4 and 5 at higher frequencies.

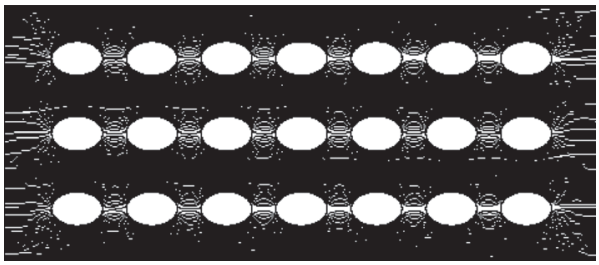

(a)

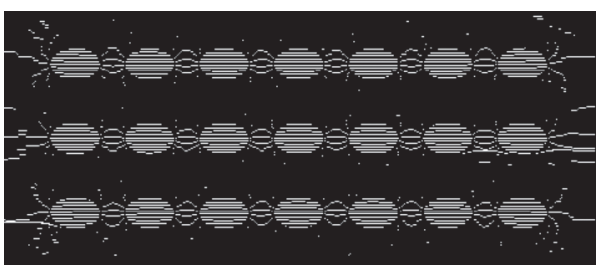

(b)

FIgURE 6: The current density at low frequency (a) is primarily distributed in the extracellular medium while at high frequency (b) goes through both the extra and intracellular medium. Simulation performed in COMSOL Multiphysics. The black and white colors indicate low and high current density, respectively.

IBC, which is in part based on the coupling of currents into the human body, can be explained according to this phenomenon of electrical conduction through biological tissues. The selection of the appropriate carrier frequency in IBC arises from a trade-off between several factors: application in use, type of signal coupling, safety regulations to avoid interference with common biological signals, specifications of 
TABLE 3: Basic ICNIRP guideline limits for time-varying electromagnetic fields.

\begin{tabular}{|c|c|c|c|c|c|c|}
\hline $\begin{array}{l}\text { Exposure } \\
\text { characteristics }\end{array}$ & Frequency range & $\begin{array}{l}\text { Current density } \\
\qquad\left(\mathrm{mA} / \mathrm{m}^{2}\right)\end{array}$ & $\begin{array}{l}\text { Internal electric field } \\
\qquad(\mathrm{V} / \mathrm{m})\end{array}$ & $\begin{array}{c}\text { Whole-body } \\
\text { average SAR } \\
(\mathrm{W} / \mathrm{kg})\end{array}$ & $\begin{array}{c}\text { Local SAR } \\
\text { (head and torso) } \\
(\mathrm{W} / \mathrm{kg})\end{array}$ & $\begin{array}{c}\text { Local SAR } \\
\text { (limbs) } \\
(\mathrm{W} / \mathrm{kg}) \\
\end{array}$ \\
\hline \multirow{3}{*}{$\begin{array}{l}\text { Occupational } \\
\text { exposure }\end{array}$} & $10 \mathrm{kHz}$ to $100 \mathrm{kHz}$ & $f / 100$ & $2.7 \times 10^{-4} f$ & - & - & - \\
\hline & $\begin{array}{l}100 \mathrm{kHz} \text { to } \\
10 \mathrm{MHz}\end{array}$ & $f / 100$ & $2.7 \times 10^{-4} f$ & 0.4 & 10 & 20 \\
\hline & $10 \mathrm{MHz}$ to $10 \mathrm{GHz}$ & - & - & 0.4 & 10 & 20 \\
\hline \multirow{3}{*}{ Public exposure } & $10 \mathrm{kHz}$ to $100 \mathrm{kHz}$ & $f / 500$ & $1.35 \times 10^{-4} f$ & - & - & - \\
\hline & $\begin{array}{l}100 \mathrm{kHz} \text { to } \\
10 \mathrm{MHz}\end{array}$ & $f / 500$ & $1.35 \times 10^{-4} f$ & 0.08 & 2 & 4 \\
\hline & $10 \mathrm{MHz}$ to $10 \mathrm{GHz}$ & - & - & 0.08 & 2 & 4 \\
\hline
\end{tabular}

very low consumption and high tissue conductivity, external noise, and so forth.

2.3. Safety Requirements and Regulations in IBC. The essence of the IBC process is the electromagnetic signal transmission in, on, or around the human body, thanks in part to the near field. Excessive electromagnetic absorption may cause unexpected harmful influence to the human organs functionality [125]. The most referenced regulations are the ICNIRP (International Commission on Nonionizing Radiation Protection) guidelines, which divide the basic limits into occupational exposure and general public exposure according to different group characteristics [22, 23]. The occupational exposure is set for occupational groups working in a controllable radiation environment, who are professionally trained to take appropriate measures to protect themselves. The general public exposure refers to the general population of different genders, ages, and health statuses who do not undergo professional training to avoid radiation, such as the elderly, women, and children. The ICNIRP defines three different physical quantities as the basic limits at different frequencies. When the frequency ranges from $1 \mathrm{~Hz}$ to $10 \mathrm{MHz}$, the main limiting physical quantity is the current density $(J)$ or electric field $(E)$, and, from $100 \mathrm{kHz}$ to $10 \mathrm{GHz}$, the main limiting physical quantity is the Specific Absorption Rate (SAR). The last quantity is the power density $(S)$ used for measured frequencies that are extremely high: $10 \mathrm{GHz}$ to $300 \mathrm{GHz}$ [22]. Note that the existing IBC research is mainly focused on the frequency range within the range from $10 \mathrm{kHz}$ to $100 \mathrm{MHz}$. Table 3 lists the basic ICNIRP limits for current density, electric field, and SAR at different frequencies under conditions of occupational and general public exposure [22, 23]. It must be noticed that the ICNIRP guidelines also emphasize that the prescribed limits are not the boundaries to distinguish safety from harm, but rather they provide upper limits of acceptable levels of protection [126].

This way, the current density should not exceed $f / 500$ or electric field should be lower than $1.35 \times 10^{-4} f$, and the SAR should be lower than $4 \mathrm{~W} / \mathrm{kg}$ in the frequency range of IBC systems, which are taken as the evaluation criteria to analyze the safety effects of galvanic-coupled weak IBC signals with different amplitudes and frequencies acting on the human arm $[22,23]$. In addition, thermal effects may also appear caused by IBC transceiver's continuous operating mode. According to the JSMEBE (Japan Society of Medical Electronics and Biological Engineering) the temperature below $42.5^{\circ} \mathrm{C}$ is safe for the tissues surrounding the electrical circuit [127]. As will be discussed in the next section, existing literature in IBC is mainly focused on the human body channel modeling with analytical and numerical methods, the distributed equivalent circuit modeling, the transceiver design, and the phantom or in vivo experiment. Therefore, the study of electromagnetic safety in IBC is still an outstanding issue. Despite this, the study of the current density distribution and potential amplitude is also involved in the study of human channel modeling. A five-layer concentric cylinder equivalent to the human arm was designed by Callejón et al. in [9], obtaining the current density distribution of each tissue layer for different frequencies and electrode distances. Lučev et al. [128] simplified the human arm to a four-layer concentric cylinder with a radius of $5 \mathrm{~cm}$ and a height of $45 \mathrm{~cm}$, and they studied the proportion of current density distribution in different tissues. The results showed that the current density in the muscle layer is the largest. Based on the special working conditions of galvanic-coupled IBC, authors of [129] simplified the human arm model to a fourlayer concentric cylinder and studied the current density distribution in each tissue layer when the muscles electrical conductivity was changed under different frequencies. Although some works have reported on the effects of the weak IBC electrical signal on the human body, few of them used the electromagnetic safety guidelines to evaluate the effects of IBC signals acting on the body. This is therefore an open issue which needs to be analyzed in greater detail.

2.4. Modeling of IBC Channels. Unlike the empirical approximations usually used in the design of IBC prototypes, mainly based on the optimization of different parameters such as data rate, consumption, position, and size of the electrodes, IBC models contribute to the design process as well as to the understanding of transmission mechanisms through biological tissues. Since IBC emerged as a promising alternative for the development of WBANs, several approaches to the modeling of the human body as a communication channel have been proposed in the literature, which can be grouped in three fundamental categories: body channel 
electric circuit models, electromagnetic models, and physical models based on phantoms. In this subsection, the main contributions made in the literature regarding these three modeling approaches are reviewed and compared.

2.4.1. Body Channel Electric Circuit Models. In this respect, the main proposals of channel models in IBC have been directed to the definition of both lumped $[24,30,33,36,38$, $130,131]$ and distributed parameters circuit diagrams $[18,35$, 37, 40, 132]. These models easily and intuitively incorporate some of the electrical characteristics of the different tissues, such as tissue resistivity and capacitive properties, as well as their dependence on frequency, thus helping obtain simple analytical expressions for both attenuation and dispersion through the human body. In addition, the distributedparameter circuit models also allow the analysis of a key parameter in IBC communication such as channel length.

Since the first model proposed by Zimmerman in [5], considerable advances have been made and circuit models have become increasingly more detailed. The model proposed by Zimmerman for capacitive coupling consisted of a simplified lumped circuit diagram in which the human body was represented as a single node, respectively, coupled with the transmitter, receiver, and ground plane through eight capacitive impedances. The signal path in this diagram is therefore established between the signal electrode of the transmitter and the receiver signal electrode, passing through the node that models the human body. This model emulated the capacitive couplings arising at this modality but did not consider the resistive properties of the human body. The model later proposed by Partridge et al. in [32] was based on Zimmerman's one-node circuit model, to which some other resistance parameters were added to emulate the resistive losses in the signal path through the human body. A more detailed model was proposed by Hachisuka et al. in [33], consisting of a four-terminal circuit model formed by six impedances, two impedances in the longitudinal path between TX and RX electrodes, two transverse impedances between electrodes of the same pair, and two other crossed impedances. Applying this model to simulate the signal transmission by the arm in the range from 1 to $1000 \mathrm{kHz}$, it was found that the gain increased considerably if the ground electrodes of the transmitter and the receiver were not placed directly on the skin but slightly separated from it. Subsequently, Wegmueller et al. improved this model by including the effect of the electrode coupling impedance to the skin, thus obtaining a four-terminal circuit model with ten impedances [24] for a galvanic IBC system between $10 \mathrm{kHz}$ and $1 \mathrm{MHz}$. This model was then developed by considering some of the bioelectric properties of cell membranes and Cole-Cole frequency-dependent dielectric properties for skin and muscle [30]. This type of circuit structure was later broadened by Song et al. [36] by adding the input impedance of the receiver device and the output resistance of the signal generator, with a total of twelve impedances. In order to emulate the attenuation through different signal paths, the torso and limbs were modeled as multilayer tissue cylinders, thus calculating the equivalent impedances according to the area and dielectric properties of the tissues considered.
More detailed circuit diagrams have later been proposed by Kibret et al. in [38], where a circuit model was reported for galvanic coupling which emulates current flow through a set of fourteen impedances modeling different layers of tissue such as skin, fat, and muscle, both in the longitudinal and in the transverse paths. A work by Swaminathan et al. in [130] proposes a lumped circuit model to simulate the galvanic IBC transmission in both on-body and in-body links from $100 \mathrm{kHz}$ to $1 \mathrm{MHz}$. The proposed model contains six impedances that account for the longitudinal transmission across each individual tissue (skin, fat, muscle, and bone), four transverse impedances emulating the current flow from one tissue to another, and four impedances that simulate the electrode-skin contact. Some of the simulated results were validated with ex vivo experiments on porcine tissue, which showed a similar adjustment with maximum deviations of $7.5 \mathrm{~dB}$ over several hours. Among the conclusions derived from the work, the authors highlighted a possible difference of up to $30 \mathrm{~dB}$ in attenuation due to the variability of tissue properties, as well as a significant improvement in channel gain when both the TX and RX devices were placed in the muscle, rather than in the skin.

Alternatively, some authors have also proposed distributed-parameter circuit models for IBC transmission. A distributed model based on the cascade of basic RC cells allows the influence of the separation between TX and RX electrodes to be analyzed. In [35], Cho et al. introduced a circuit model formed by the cascade of distributed RC blocks simulating the trunk and the arms together with a coupling capacitance to the external ground. The frequency range of study was from $100 \mathrm{kHz}$ to $150 \mathrm{MHz}$. The simulations showed a behavior of the human body as a high pass filter below $4 \mathrm{MHz}$. However, as frequency increased, the deviations between experimental results and simulations became significant, especially at greater distances. In [37], Callejón et al. proposed a distributed circuit scheme based on a transmission line model using infinite periodic structures. This model was formed by the cascade of basic electric cells along a longitudinal axis between TX and RX electrodes. The objective of this type of structure is to study the influence of an admittance that is repeated in the transmission path of a signal. This admittance modeled electrophysiological properties of the skin; in particular, a conductance simulated the conductive pathways, sweat glands, and ionic channels of the cell membranes, and a susceptance emulated the insulating behavior of the keratinized cells of stratum corneum. An improved model was later proposed in [132], obtaining a relationship between the propagation constant of the medium, in this case the skin, with different transverse impedances depending on the coupling type. This way, the same model was adapted to the study of both galvanic and capacitive techniques. Pereira et al. proposed in [18] a circuit diagram for capacitive coupling considering the effect of the cables and devices used in the measurements, which introduce nonlinearities and parasitic effects that can significantly affect body attenuation. The complete scheme consisted of a distributed-parameter circuit model emulating both the intrinsic path through tissues, formed by the cascade of RC cells emulating the current pathways through the arm, chest, and torso; and a lumped 
circuit emulating the extrinsic path formed by the capacitive couplings between the ground electrodes and other external elements such as wires and baluns. More recently, in [40], Wang et al. proposed a cascade network body capacitive channel model composed of a series of $10-\mathrm{cm}$ unit RC body cells emulating the skin dielectric properties. The derivation of matrix cascade networks simplifies computation and provides useful analytic tools for the analysis of performance evaluation and transceiver design optimization.

2.4.2. Electromagnetic Models. The electromagnetic (EM) modeling of IBC communication comprises two different approaches: the resolution of Maxwell's equations through numerical techniques such as finite-difference time-domain method (FDTD), finite element method (FEM), or method of moments (MoM) and the theoretical modeling of electromagnetic propagation by means of simple formulas and geometries that allow analytical expressions in the body medium to be obtained.

One key parameter in IBC is the human body attenuation. In the computational studies addressed in the literature, factors such as the influence of the geometry and the dielectric properties of living tissues have been investigated, together with the optimal configuration of the electrodes to achieve a higher signal level at the RX site. One of the first computational studies was presented by Fujii et al. in [41], where a human arm was simulated as a parallelepiped with dielectric characteristics equal to those of muscle tissue at a frequency of $10 \mathrm{MHz}$ using FDTD technique. They found that if the TX ground electrode was in contact with the skin, the electric field in the RX site was strengthened. However, if the ground electrode of the RX was in contact with the skin, the received signal level decreased. The same authors later presented in [42] a high resolution FDTD model of two Japanese adults, of both sexes, with which they studied the electrical field distribution in different areas of the body and mainly through the arm, where they found that most of the electric field was confined. The model proposed by Hwang et al. in [43] presented a U-shaped geometry to emulate the two arms and shoulders, formed by the superposition of five tissue layers (skin, fat, muscle, and cortical and cancellous bone). The signal losses were simulated in a total of five frequencies between 10 and $50 \mathrm{MHz}$ considering several configurations with and without a second ground electrode attached to the arm. The results showed that it was not necessary to place the transmitter ground electrode in contact with the skin for transmission distances along the arm smaller than $15 \mathrm{~cm}$. In fact, the device's consumption decreased with this configuration. However, for longer distances, the ground electrode was placed in contact with the subject's skin to improve channel gain. Wegmueller et al. presented a $3 \mathrm{D}$ FEM cylindrical model of the arm conformed by the five previously mentioned layers, with which the influence of the channel length, the size of the electrodes, and the effect of the joints were studied [34]. Their simulations showed the feasibility of IBC communication in galvanic mode at distances of 5,10 , and $15 \mathrm{~cm}$. They found that an increase of $5 \mathrm{~cm}$ in channel length caused an increase of up to $9 \mathrm{~dB}$ in attenuation. They also obtained an increase in attenuation of up to $8 \mathrm{~dB}$ due to joints. A similar cylindrical FEM model was developed by Lučev et al. in [128], analyzing the current distribution through the human arm at three different frequencies $(100 \mathrm{kHz}, 1 \mathrm{MHz}$, and $10 \mathrm{MHz})$. The ratio between the capacitive and the resistive current components and the influence of parameters such as skin moisture and electrode size were also studied. At these frequencies, it was seen that most of the current density flows through the muscle tissue and that this increased with frequency, skin moisture, and electrode size. Other authors have also considered the influence of trunk and limbs on their computational models. In [19], Xu et al. proposed a FEM model of the human body for capacitive coupling in which the trunk was modeled as a parallelepiped and the limbs as a cylinder formed by several concentric tissue layers. The channel showed a high pass response through the capacitive return path. A study presented in [46] by Song et al. proposed a FEM model of the whole human body for galvanic coupling, presenting the distribution of electric potential in different body areas for ten frequencies in the range from $10 \mathrm{kHz}$ to $5 \mathrm{MHz}$. The human body was represented as a set of cylinders to emulate the head, neck, limbs, and torso. These cylinders were formed by four layers of tissues comprising skin, fat, muscle, and bone. Different signal paths were studied by placing the electrodes in different positions along the same arm, from the arm to the torso, from the arm to the head, and so on. One of the conclusions drawn by the authors is that the separation between electrodes does not have a great effect on channel attenuation from 10 to $100 \mathrm{kHz}$, starting to take on relevance from $500 \mathrm{kHz}$ to $5 \mathrm{MHz}$. In addition, they concluded that the muscle has no great influence on the distribution of the electric potential, which is mainly confined in the upper layers, that is, skin and fat. Callejón et al. proposed in [9] a FEM model (see Figure 7) of the human arm formed by a multilayer cylindrical geometry composed of five concentric tissues such as skin, fat, muscle, and cortical and cancellous bone. The study covered a wide range of frequencies between $1 \mathrm{kHz}$ and $100 \mathrm{MHz}$. In order to emulate the properties of permittivity and conductivity of human tissues in this band, four-dispersion Cole-Cole models were considered. The simulations comprised results of the bioimpedance of the arm, the electric field distribution both inside the tissues and on the external surface near them, and the distribution and the percentage of current flowing through each tissue as a function of variables such as frequency, channel length, and interelectrode distance. Figure 8 shows simulated results obtained with this model. In addition, simulations of specific body attenuation as a function of these two last variables were obtained, which were validated with experimental measurements. The results showed that the attenuation in the galvanic IBC mode is strongly dependent on channel length, increasing up to $20 \mathrm{~dB}$ for increments in distance of $5 \mathrm{~cm}$. It was also found that attenuation is dependent on the transverse length between electrodes of the same pair, obtaining better levels of received signal by considering longer interelectrode distances. Similar computational models have later been proposed to analyze the effect of the parasitic return path [131], the influence of environmental noise [133] and real experimental conditions [134], the differences 


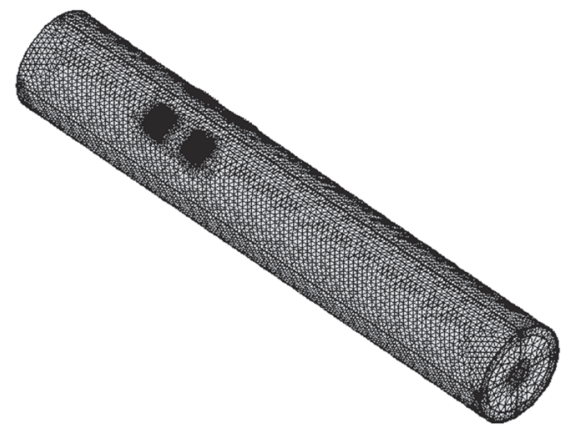

FIGURE 7: Image of the FEM model proposed in [9] consisting of a multilayer 3D cylinder composed of concentrical tissues such as skin, fat, muscle, and cortical and cancellous bone.

between surface-to-surface and implanted communications [130], and the use of noncontact electrodes at the transmitter [131]. A hybrid model considering both electrostatic circuit analysis and FEM model simulations has been proposed in [50] by Park et al. to analyze different parameters of HBC channel such as the external coupling characteristics between the environment and the electrodes as well as the transmission performance obtained with different experimental setups comprising VNAs and miniaturized batterypowered wearable transceivers. Recently, in [135], a cylinder numerical model based on that reported in [9] has been used to perform a parametric computational study of different key IBC parameters such as the electrode-skin impedance, the transmitted signal based either on a voltage or an electric current excitation mode, the influence of anthropometrical characteristics such as arm diameter and tissue thicknesses, and the influence of bioelectric properties such as muscle conductivity and tissue anisotropy.

The main proposals for the EM theoretical modeling of IBC communication are based on the definition of basic geometries such as the cylinder and the plane, in which Maxwell's equations can be solved analytically. For example, in $[136,137]$, Gao et al. studied the electric current through the arm for a model based on a cylindrical geometry of $30 \mathrm{~cm}$ of length and $5 \mathrm{~cm}$ of diameter for galvanic coupling. A crosssectional plane of this cylinder formed by two homocentric mediums with different conductivity properties, respectively, representing the muscle and the skin, was considered. The frequency of work was lower than $200 \mathrm{kHz}$, which is the reason why the permittivity was considered negligible. It was assumed that there was no source of charge inside the section and that conductivity was constant in the frequency range studied, in such a way that the formulation of the problem was reduced to Laplace's equation by quasi-static approximation. The distribution of current density seemed to be concentrated around the electrodes. A later approximation was proposed by the same authors by adding a layer of bone tissue, showing lower influence on signal transmission. In [138], Pun et al. improved this initial model presenting a $3 \mathrm{D}$ cylindrical multilayer model emulating the channel attenuation in the human arm. In [47], Haga et al. proposed the resolution of a problem of boundary conditions for the electrical potential in the human body, modeled as a conductor with losses by assuming the quasi-static approach. The authors then proposed an equivalent electric circuit whose parameter values were derived from the MoM. Finally, this approach was validated with an FDTD computational model based on a rectangular geometry emulating the human arm. Bae et al. presented in [17] an approximation to the theoretical analysis of IBC communication by means of surface waves considering three components: the near-field electrostatic, the inductive, and the far field components, depending on the frequency and channel length. The study was based on the Norton method for propagation on flat earth, with channel dimensions adapted to those of the body limbs. The same authors in [48] use the same model applied to the study of different electrode configurations, both in a horizontal and in a vertical position with respect to the longitudinal axis of the human arm. In this way, they obtained a theoretical expression for the transmission gain of a total of nine possible configurations. In [49], Teshome et al. proposed an analytical EM model for IBC galvanic-coupled implanted systems. They analytically solve Maxwell's equations under quasi-static assumptions by applying Lamé's functions in an ellipsoidal geometry emulating different parts of the body such as head, torso, and limbs. They use the model proposed to analyze the influence of diverse factors in the IBC implanted channel link, such as the effect of frequency, implant size, electrode separation, and tissue conductivity.

2.4.3. Phantom Models. For the study of the propagation and the effect of electromagnetic fields on the human body, the authors have used experimental prototypes based on liquid and/or solid containers with dielectric properties similar to those of body tissues. These prototypes are called phantoms. The substances more commonly used are water and sodium chloride to model the interstitial fluid and other chemical compounds such as agar for membrane tissues. Fujii et al. proposed in [42] a solid phantom to emulate the human arm by means of a rectangular parallelepiped with a relative permittivity of 81 and a conductivity of $0.062 \mathrm{~S} / \mathrm{m}$, similar to those of the muscle at $10 \mathrm{MHz}$. Although the relative permittivity reported by Gabriel et al. for the muscle at that frequency is 170.73 , the authors found that the signal received in both cases (using the phantom and the human arm) was approximately the same. The reason for using a phantom with a lower permittivity is the difficulty to find body phantoms capable of emulating tissues with high relative permittivity. Wegmueller et al. presented in [51] an ellipsoidal phantom for the simulation of a cross-section of the torso, filled with a muscle simulating fluid called MSL27. This liquid had the property of presenting the same conductivity as the muscle at $27 \mathrm{MHz}$. Gao et al. proposed an experimental setup in [136] based on a circular phantom formed by two homocentric sections filled with two different substances: water with a certain sodium solution and agar. This phantom modeled a transverse slice of the arm, with $30 \mathrm{~cm}$ of diameter and $1 \mathrm{~mm}$ of thickness. Agar was used to emulate the skin and a saline solution accounted for the interstitial fluid and muscle. The results of the voltage and current distribution in 

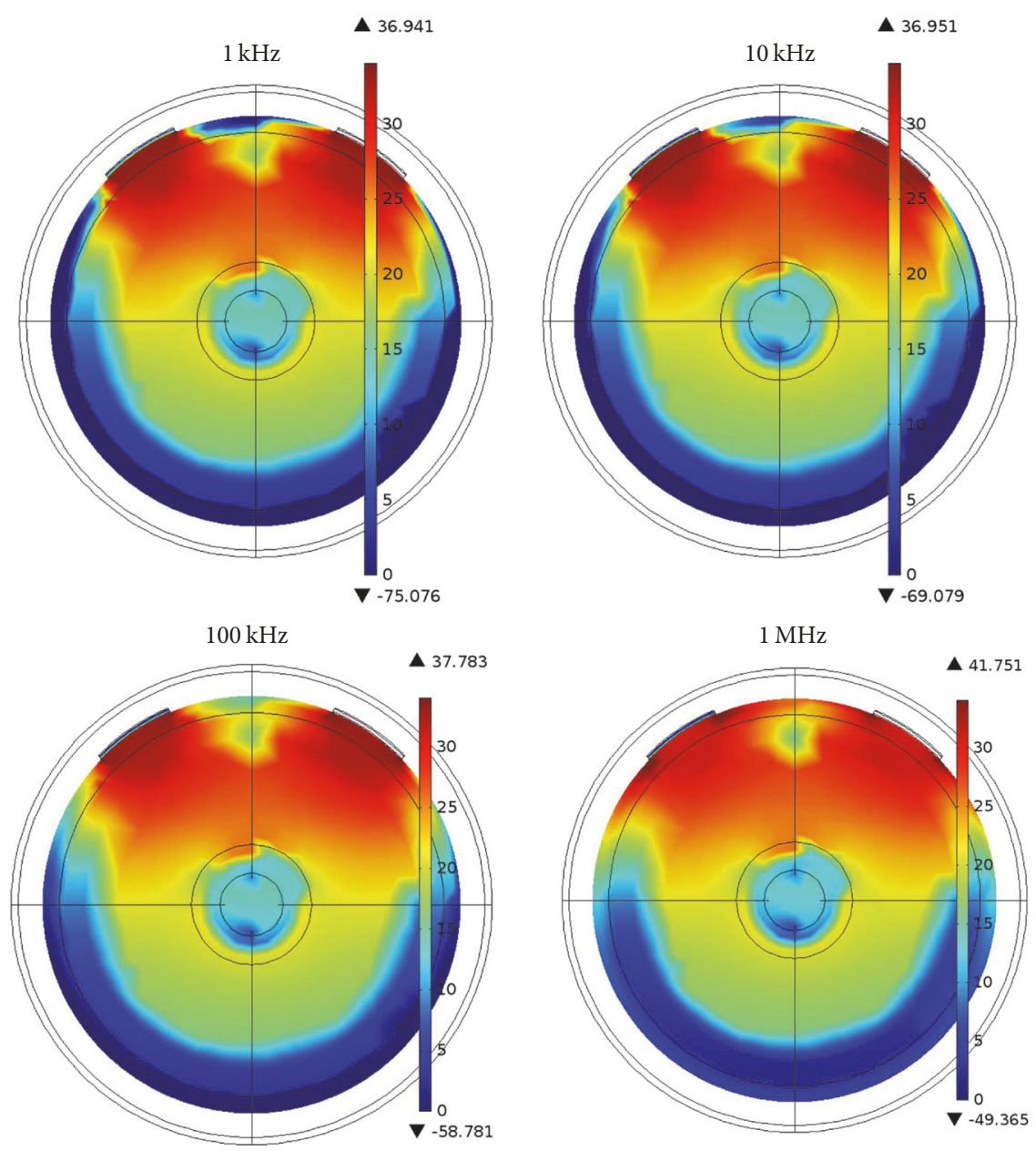

FIGURE 8: Normalized current density distribution in $\mathrm{dB}$ units through a cross-sectional plane of a cylinder emulating the human arm tissues, for an injected current of $1 \mathrm{~mA}$ at four different frequencies of $1 \mathrm{kHz}, 10 \mathrm{kHz}, 100 \mathrm{kHz}$, and $1 \mathrm{MHz}$. Simulated results derived from model in [9].

the phantom fitted the results obtained with the theoretical model proposed in the same work in a frequency close to $200 \mathrm{kHz}$. Later, the same authors in [139] proposed a phantom consisting of a semicylindrical container formed by two homocentric layers composed of different chemical compounds emulating the skin and muscle. Recently, in [52], Callejón et al. proposed a circuital phantom for the study of the influence of the experimental setup on the IBC measurement. In this way, they considered a simplified circuit of four terminals to emulate the flow of the longitudinal and transverse current pathways through tissues in galvanic coupling. The circuit phantom was designed to simulate the bioimpedance and the attenuation of the arm, previously obtained by the authors using a FEM model in [9]. In this way, the phantom circuit was a simple and direct way to perform different tests with different experimental setups, avoiding the inherent uncertainty associated with the human body itself and the difficulty of reproducing identical conditions between different experimental sessions. In [49], Teshome et al. developed a homogeneous aqueous muscle tissue phantom at a frequency of $13.56 \mathrm{MHz}$ to corroborate the simulated results derived from both an analytical and an FDTD model to study the effects of different key parameters in IBC galvanic implanted systems.

2.4.4. Comparison and Discussion. Table 4 summarizes some of the IBC models described in the previous subsections, according to diverse characteristics such as the modeling approach, type of coupling, and frequency range. An efficient use of the human body as a transmission medium entails the understanding of the underlying signal transmission properties through living tissues. However, this is not a straightforward task. Each modeling approach has advantages and disadvantages, making a trade-off between accuracy and complexity necessary. The simplicity of the electric circuit body models in order to obtain useful analytic expressions for body attenuation justifies their use to guide the design of IBC transceivers and systems. Compared with numerical models, which usually present high computational cost, the electric circuit body models offer a compromise between 


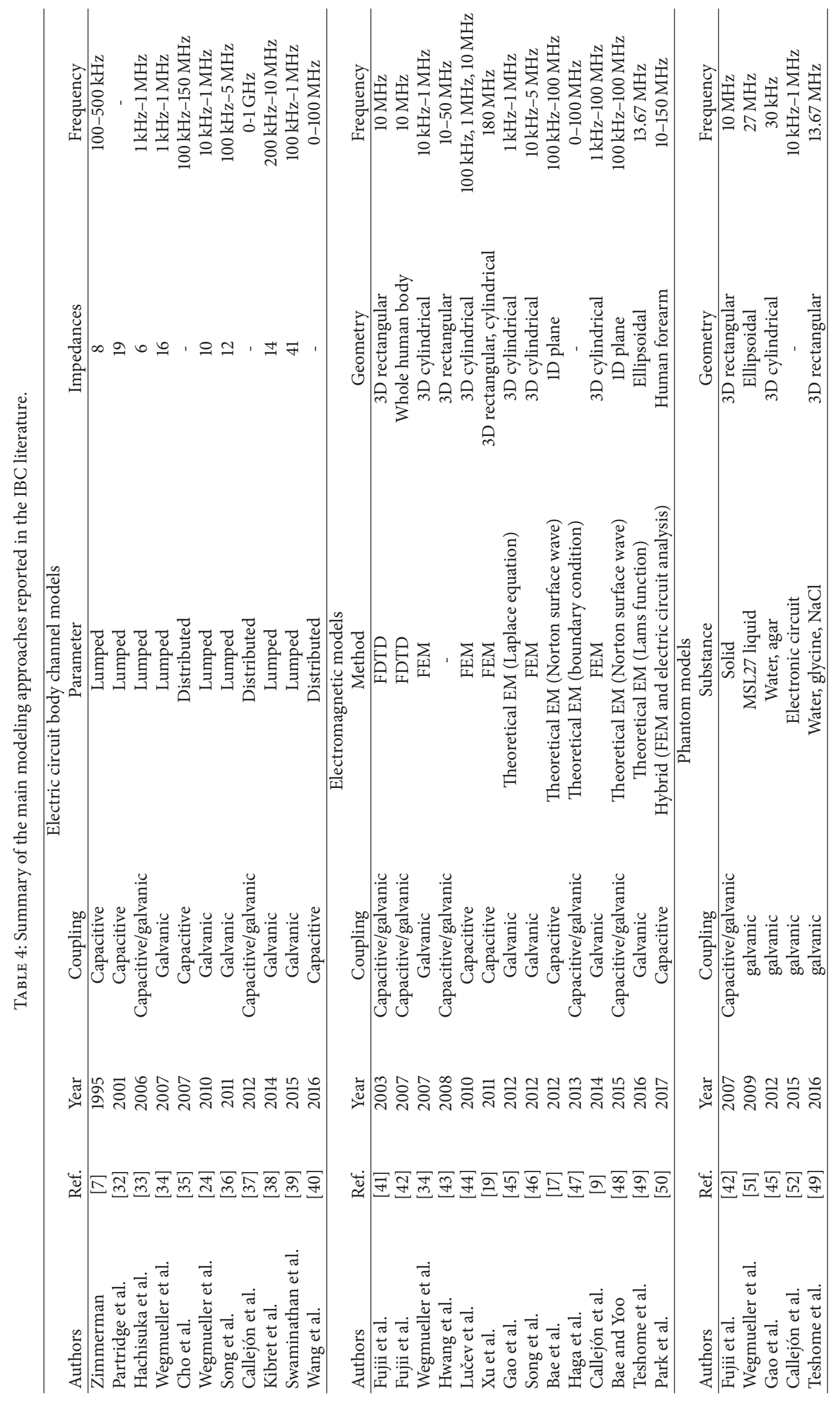


simplicity and precision. However, their main limitation is that as frequency increases, other effects such as radiation from cables and electrodes, the body antenna effect, and other signal dispersion phenomena become nonnegligible. These effects are no longer emulated by this type of electric circuit models; therefore their validity is usually constrained to low and medium frequency bands. Since the first model proposed by Zimmerman in 1995 [7] formed by eight capacitances to emulate the capacitive couplings between the body and the external ground, models have evolved towards complex RC networks emulating different tissue layers at both longitudinal and transversal directions [38-40], thus taking into account the frequency-dependent dielectric properties of tissues [34, 46], the cross-impedances between TX and RX electrodes [33], the output and input resistance of the TX and RX device [36], and so forth.

Electromagnetic models, both analytic and computational approaches, have attracted more and more the attention of IBC researchers giving rise to a variety of models ranging from simple geometries such as plane, cylinder, and parallelepiped, which emulate parts of the body such as limbs and trunk $[9,41,46]$, to models based on the anatomy of the arm and the whole human body [42, 50]. However, there is still a lack of simple analytic expressions that can be used to systematically and accurately guide the design process. Theoretical models easily allow the understanding of basic principles of signal transmission through human tissues, but the simple geometries considered such as plane and cylinder only represent a first approximation to a phenomenon where the effects of a complex anatomy can be the cause of the discrepancy observed between experimental and simulated results. However, the necessity of considering more complex geometries implies the use of numerical methods such as FDTD and FEM. These allow complex anatomy and anthropometrical characteristics to be analyzed, at the expense of time, computational cost, and a high amount of data. Furthermore, it must be said that computational models are difficult to reproduce, since they usually depend on the simulation characteristics and the numerical approach adopted.

Physical models based on phantoms of the human body allow experimental measurements to be carried out without temporal or physical limitation, in scenarios where experimentation with living beings would be highly invasive. In addition, they can be implemented in a relatively simple way, choosing the appropriate substances and liquids to model the body tissues of interest. On the other hand, the main limitation they present is associated with the difficulty to find liquids or substances that emulate the electrical properties of human tissues in a wide range of frequencies. Therefore, these models are partial and not very versatile for multifrequency studies.

In this subsection we have discussed and compared the different modeling approaches presented in the literature, concluding that each of them has its own characteristics and range. Possibly, future advances in IBC modeling will require a combination of them: a first electromagnetic theoretical model to obtain closed analytic expressions that contribute to knowledge, along with a second more complex computational model that helps quantify the deviations and the percentage of error with respect to the first, as well as the influence of the complex anatomy of the human body. Finally, and based on these two proposals, a circuit model is usually the most common approach to obtain basic rules for the design of IBC transceivers. In addition, it is worth highlighting the fact that the resolution method is highly dependent on the application that is going to be developed. Thus, the IBC applications based on galvanic and capacitive coupling at low frequencies can be analyzed by means of circuital models that allow the voltage and electric current magnitude to be determined at both TX and RX sites. In this case, the signal is electrically coupled to the human body, and the radiation component is practically nonexistent. These are IBC applications at low frequencies in which the signal is transmitted and received by means of electrodes placed on or very close to the body and the quasi-static approximation is still valid. However, as frequency increases, propagation begins to occur through surface waves and the electrode is relegated by the "electrode-antenna" concept, making an analysis of the propagation with more detailed electromagnetic models necessary.

\section{Measurement Issues}

This section comprises some of the more relevant measurement issues in IBC field, respectively, electrodes and body coupling issues, the main experimental setups proposed in the literature to characterize the human body channel, the study of the influence of the anthropometric characteristics and movement of the users, and the main sources of artifacts and noise in IBC.

3.1. Electrodes and Body Coupling Issues. The electrodes in IBC are analogous to antennas in airborne wireless communication systems [140]. This is why they play a very important role in IBC communication. Several authors have studied the influence of their properties on channel attenuation [38, 77]. In fact, it has been found that the electrodes represent a source of uncertainty in the measure, since they present highly variable properties, such as the dispersion shown in its impedance values, as well as noise and adhesion problems [135]. Different results have been obtained by researchers, depending on whether they apply galvanic or capacitive coupling.

In galvanic coupling, electrodes provide an electrochemical interface between the IBC transceiver and the human body, both at the TX and at the RX sites. Hachisuka et al. [62] studied the influence of the size of square electrodes, from 10 to $50 \mathrm{~mm}$ length. In the frequency region near $10 \mathrm{MHz}$, they found a maximum in gain for $25-\mathrm{mm} \times 25$ $\mathrm{mm}$ square electrodes, showing that the gain decreased for larger or smaller sizes. This may be due to a decrease in the electrode capacitance as it becomes smaller and an increase in current leakage between electrodes of the same pair as they become larger. Other authors have studied the influence of electrode-skin contact. According to [141], the contact impedance between the electrode and the body is independent of the type of metal (aluminium, copper, bronze, 


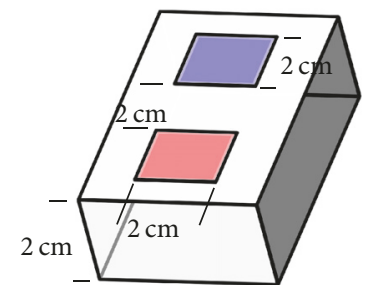

Electrode pair A

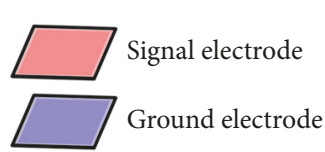

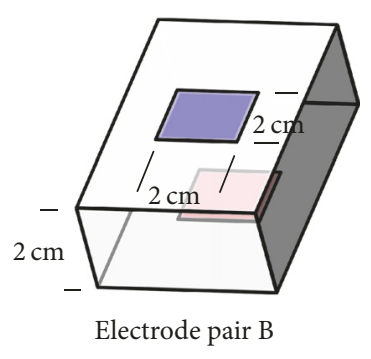
to

FIGURE 9: Example of capacitive electrodes [10]: signal and ground electrodes are fixed on the same side of the PVC strip (electrode pair A) and copper areas one above the other (electrode pair B), oriented towards the opposite directions.

brass, stainless steel, nickel silver, and silver/silver chloride $(\mathrm{Ag} / \mathrm{AgCl}))$. The results of Callejón et al. [29] comparing different types of electrodes (pregelled $\mathrm{Ag} / \mathrm{AgCl}$, aluminium, and copper) confirmed that the electrode material does not bear too much influence on IBC performance whenever a conductor is used. However, $\mathrm{Ag} / \mathrm{AgCl}$ electrodes proved to have better performance even though their active area is the smallest, which could be explained by the fact that they are pregelled, thereby enhancing both the conductive properties and the adherence to the skin. In fact, because of the gel, the electrode-skin impedance is lower than that of the rest of electrodes, and, therefore, a greater current is injected for the same voltage, causing higher signal levels to be received. They also observed that pregelled electrodes were more robust against movement artifacts. According to Kibret et al. [142], the increase in gain at high frequency can be due to the decrease of the skin impedance, dominated at lower frequencies by a strongly resistive stratum corneum. The impedances of other components, like muscle and fat, are approximately constant in the frequency range from $100 \mathrm{kHz}$ to $10 \mathrm{MHz}$. Okamoto et al. examined four types of electrodes: a stainless-steel electrode, a titanium electrode, and a dry and a pregelled $\mathrm{Ag} / \mathrm{AgCl}$ electrode. Their results suggest that electrodes showing lower electrode-skin resistance properties improve IBC transmission. Regarding the interelectrode distance (between the signal and ground electrodes), several values have been analyzed in the literature, from $1.4-\mathrm{cm}$ to $7-$ $\mathrm{cm}[10,46,52,82,95,143,144]$. A distance of 9 -cm was chosen in the galvanic coupling experiments carried out by Callejón et al. [132], since it was found that the longer this distance is, the lower the attenuation results are.

In capacitive coupling, different configurations of electrodes have been analyzed. In [10], two configurations of capacitive electrodes were compared, with only the signal electrode in direct contact with the skin (see Figure 9), although other electrodes configurations can also been considered [11] (see Figure 10). In this coupling mode, the received signal is affected by the orientation of the transmitter with respect to the receiver, the size and type of the electrodes, the distance of ground electrodes to the external ground, and the surrounding environment [145]. Callejón et al. [29] evaluated different types of signal electrodes (pregelled $\mathrm{Ag} / \mathrm{AgCl}$, aluminium, and copper) and their influence on the signal attenuation. The results showed a similar trend with frequency. However, copper electrodes showed better performance up to $60 \mathrm{MHz}$, and, specifically, a difference of $10 \mathrm{~dB}$ in magnitude level was found. Three different electrode types were tested in [146], with minimal influence on the measurement results: bare copper electrodes without a conductive paste, copper electrodes with a conductive paste, and standard self-adhesive $\mathrm{Ag} / \mathrm{AgCl}$ electrodes with the conductive paste. The size of the electrodes has also been studied in capacitive coupling. According to Zhao et al. [147], the attenuation decreases when the ground electrode is larger but is not affected by the size of the signal electrode when it contacts the body directly. In the same direction are the results obtained by Callejón et al. [29]. They evaluated different sizes of the ground electrode $(3-\mathrm{cm} \times 3-\mathrm{cm}, 4-\mathrm{cm} \times$ $4-\mathrm{cm}$, and $7-\mathrm{cm} \times 7-\mathrm{cm})$, finding that the greater the size of the ground electrode, the greater the signal received [29]. Using a large ground plane or a special electrode for the return path loop is advantageous to enhance the signal-tonoise ratio (SNR) of the received signal [35]. However, this goes against the miniaturization of a communication device, which means that a trade-off must be sought. Since a large ground plane is difficult to implement on the body channel transceiver, it is useful to find a lower limit on the plane size. Cho et al. [35] proposed an empirical formula for the minimum ground size. Notice that the ground electrode is usually connected to the ground level of the electrical circuit [42]. Besides, greater distance between the signal electrode and the ground electrode reduces the capacitance between them and induces more energy in the body [147].

Additionally, some adjustments can be made to the electrodes to optimize the communication performance. In Ohishi et al. [148], capacitive electrodes are enhanced by inductance coils so that the resonance frequency is equal to the IBC frequency when the electrodes are close to human body surface. Impedance-matching networks at the transmitter output and the receiver input can also increase the transmission performance in capacitive coupling $[12,40]$ (see Figure 11).

Galvanic and capacitive coupling performance has also been compared from the point of view of the electrodes. Alshehab et al. [141] investigated the optimal configuration by considering inclusion and exclusion of a ground electrode touching the skin, different sizes of electrodes, ground circuit board, and the distance between the signal electrode and the circuit board. They concluded that the best configuration, in terms of the lowest path loss, was composed of only signal electrode touching the skin (round copper plate with $2-\mathrm{cm}$ of diameter) and a $10-\mathrm{cm} \times 5-\mathrm{cm}$ ground circuit board at a distance of $1.5-\mathrm{cm}$ from the signal electrode. Similar results were obtained by Ruiz et al. [149] and Grilec et al. [70]. However, according to [47], the signal quality is raised up by attaching both the signal and the ground electrodes to the human body, so the electric field and the received voltage can be enhanced. Fujii et al. [42] concluded that existence of the 

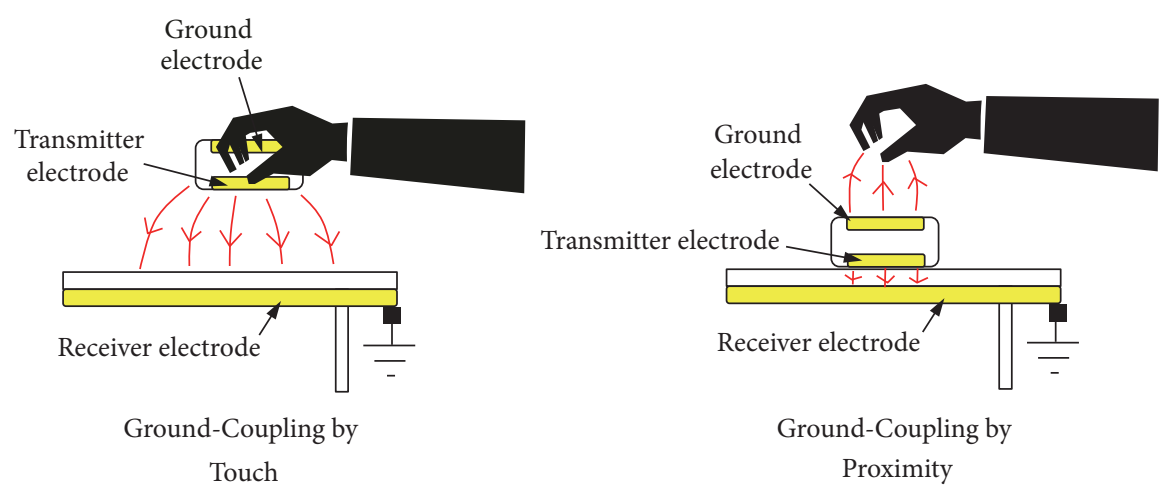

Ground-Coupling by Proximity

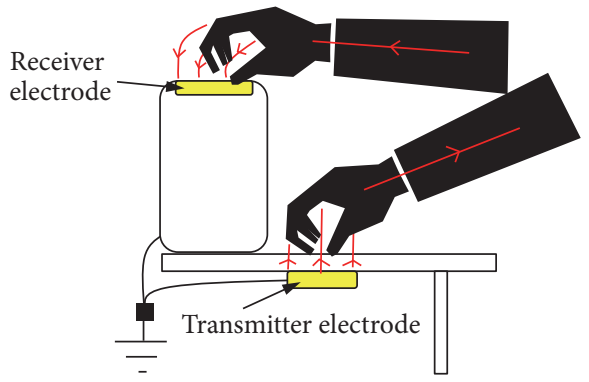

Ground-Coupling with Common Ground

FIGURE 10: Modes of capacitive coupling according to [11]: Ground-Coupling by Touch. The RX device is connected to the external ground, and the TX is a battery-powered device. Ground-Coupling by Proximity. A RX is connected to the external ground, and a battery-powered smart device is close to the RX. Ground-Coupling with a Common Ground. Both devices are directly connected via a common ground and an information flow can be established through the user's body.

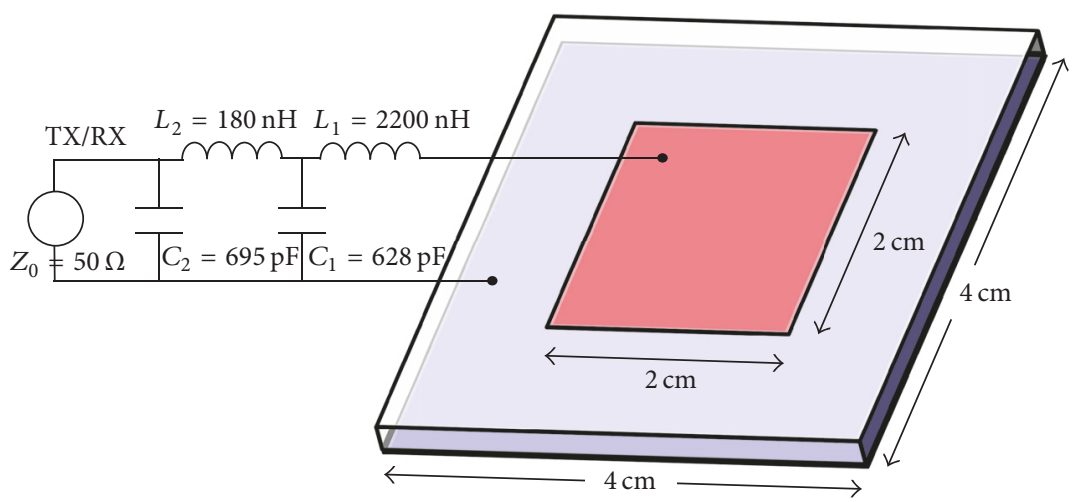

FIGURE 11: Schematic of the matching network proposed in [12].

ground electrode can be quite effective in transmitting the signal because it enables the impedance matching between the signal generator and the human body [42]. In both capacitive coupling and galvanic coupling, it has been shown that the attenuation of the body channel can be much lower than that of the air channel in frequencies up to $100 \mathrm{MHz}$ $[68,150]$ (below $84 \mathrm{MHz}$ according to Seyedi and Lai [151]). In galvanic coupling the received signal has less dependence on the environment [145]. On the other hand, the results of Hachisuka et al. [33] indicate that capacitive coupling is more suitable for high-speed IBC devices than galvanic coupling. The same results were found in [152].
In summary, there is no uniformity in the use of electrodes for IBC systems and varied sizes and materials have been proposed. Copper has been used as the electrode material in many studies because of its good conductivity [141]: $25-\mathrm{mm} \times 25-\mathrm{mm}$ [153], $3-\mathrm{cm} \times 3-\mathrm{cm}$ [132], $2-\mathrm{cm} \times 2-$ $\mathrm{cm}[20,40,152,154,155], 6-\mathrm{cm} \times 8-\mathrm{cm}[156]$, or circular with a radius of $10-\mathrm{mm}$ [36]. Stainless-steel electrodes of $5-\mathrm{mm} \times$ $15-\mathrm{mm}$ was employed in [157]. Asogwa et al. used [143] selfadhesive silver/silver-chloride electrodes $(\mathrm{Ag} / \mathrm{AgCl})$ because they are designed for both research and clinical use, contain hypoallergenic gel, can be used for two hours of measurement, and reduce the effects of motion artifacts. The same 
electrode type was employed in $[16,24,27,68,95,142,152$, 158].

\subsection{Measurement Issues and Experimental Characterization.} The experimental characterization of the human body as a communication channel has been shown to be strongly dependent on the environmental conditions and measurement methods under which the experiments are performed, leading to important deviations between results reported by some authors and others, thus not allowing a formal comparison to be drawn. There is also a heterogeneous set of proposals to address different technical issues, for example, various strategies for the isolation of the internal ground from electronic equipment, differential signaling, electrodeskin impedance matching, and so forth, giving rise to a wide variety of experimental schemes and measurement setups. Likewise, the reported measurements have been made using a wide variety of electrodes of different shapes, materials, and sizes, as highlighted in Section 3.1. With respect to the body areas studied, most of the published results have been obtained in the human arm, with some works also reporting measurements in other parts of the body such as legs, back, torso, and head $[153,159]$. Regarding operating frequencies, there is no consensus about the optimum frequency band for IBC communication in its two main categories of galvanic and capacitive coupling, in such a way that there is no common design methodology for IBC transceivers, which often present different operating frequencies. On the other hand, it is also worth noting that the IBC channel depends on the electrophysiological and anthropometric properties of the subjects under study. In fact, there is an implicit dependence on the body position and movements of the subject, which makes the measurement process even more difficult, leading to variations throughout different experimental sessions. The experimental setups proposed in the literature have been developed to perform measurements to analyze key issues such as optimal frequency bands, electrode type, channel length, and interelectrode distance, as well as different body parts and postures of the subject.

On the other hand, it must also be noticed that there is an important source of discrepancy in IBC measurements which has not sufficiently been studied in the literature and is in turn related to the effects produced by the electronic devices and experimental conditions. Thus, experimental schemes using different equipment such as oscilloscopes and signal generators, spectrum analyzers [48, 160], and vector network analyzers (VNA) [161], as well as customized transceivers, have been used interchangeably [144, 162]. Recently, in [50], an exhaustive study about optimal measurement configuration for HBC channel characterization using miniaturized battery-powered transceivers has been reported by Park et al. In addition, another important issue in the characterization of IBC channels is the determination and quantification of environmental noise, as modeled in [133]. It is therefore necessary to harmonize and clarify the proposed setups and measurement procedures to establish an experimental methodology that allows the characteristics of the IBC channel to be identified in a systematic and accurate way. Accordingly, some technical aspects that need to be studied with greater detail are those that relate, on the one hand, to the isolation of the internal ground of the measurement circuits, which are often parasitically coupled to the experimental scheme, giving rise to configurations that do not correspond to real capacitive and galvanic schemes, and, on the other hand, to the mismatching of the internal impedances of the equipment and that of the skin-electrode impedance [163]. Regarding this issue, an important source of disparity might be due to the input resistance of the measuring devices and transceivers used. In the literature, devices with input resistances of $1 \mathrm{M} \Omega$ and $50 \Omega$ [29, 38, 139] have been considered indistinctly. In the specific case of galvanic coupling, the impedance seen before and after the measuring device is that of the electrode-skin interface, which is frequency dependent $[9,38,140,164,165]$. Therefore, the use of electronic equipment with input resistances of $50 \Omega$ may not be an optimal option since an impedance mismatch with respect to that shown by the skin could exist, therefore leading to lower values of received signal and channel gain. Figure 12 shows a set of attenuation measurements performed to the same subject with the same experimental conditions by varying the measurement devices used at both TX and RX sites. The discrepancy due to the use of one setup to another is highlighted.

\subsection{Influence of Anthropometric Characteristics and Position} Issues. In IBC communication many overlapping physical mechanisms occur at the same time, making channel characterization and measurements a challenging task. Besides different measurement setups and IBC coupling approaches, described in previous sections, anthropometric characteristics and body positions of test subjects have been suggested as the main causes of the differences between the measurement results found in the literature $[29,38,146,166]$.

Hachisuka et al. analyzed optimal placement of proprietary capacitive IBC transmitters and receivers for different body positions and distances [33]. The two-electrode arrangement, in which only the signal electrode is attached to the body, was found to be $20 \mathrm{~dB}$ better in the kilohertz band than the four-electrode arrangement in which all four electrodes are in contact with the body. In [65] authors experimentally characterized the body-coupled (i.e., intrabody) communication channel using a specifically designed measurement system in the frequency range from $100 \mathrm{kHz}$ to $60 \mathrm{MHz}$. They showed that the maximum propagation loss for the whole body channel was below $80 \mathrm{~dB}$. The frequency dispersion and the influence of body movement on channel attenuation were shown to be much smaller than that for RF wireless body area network channels. They performed measurements on a test subject while sitting on a chair, standing, walking through the room, and standing while moving the right arm up and down. The measured body movements were shown to result in only small variations in channel attenuation, and the maximum observed standard deviation was $2.5 \mathrm{~dB}$. In [167] the authors proposed a statistical model for the intrabody propagation channel based on experimental data while the user was both still and walking, in terms of most fitting probability density function. In $[24,34,93]$ the authors performed measurements on 20 test subjects and showed 


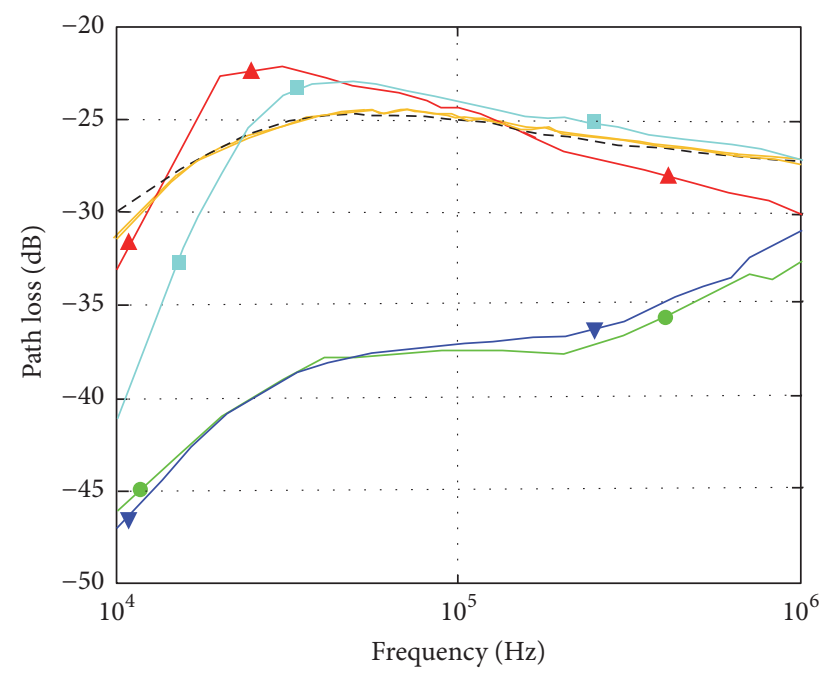

- (TX: signal generator + balun) (RX: balun + grounded oscilloscope $\left.\left(R_{L}=1 \mathrm{M} \Omega\right)\right)$

$\neg$ (TX: signal generator + balun) (RX: balun + grounded oscilloscope $\left.\left(R_{L}=50 \Omega\right)\right)$

- - (TX: grounded spectrum analyzer + balun) (RX: balun + grounded spectrum analyzer $\left.\left(R_{L}=50 \Omega\right)\right)$

- - (TX: portable current generator) (RX: portable oscilloscope $\left.\left(R_{L}=1 \mathrm{M} \Omega\right)\right)$

- (TX: portable current generator) (RX: balun + grounded oscilloscope $\left.\left(R_{L}=1 \mathrm{M} \Omega\right)\right)$

— (TX: portable current generator) (RX: grounded oscilloscope $\left.\left(R_{L}=1 \mathrm{M} \Omega\right)\right)$

FIgUre 12: Path loss obtained for a single user under the same experimental conditions varying the measurement devices at both TX and RX sites. Varied electronic equipment covering signal generators, oscilloscopes, spectrum analyzers, and portable customized current generators, which present distinct input resistances, has been used with the aim of showing the deviation in attenuation measurements.

that the more body activity there is, the better the galvanic coupling is and the more stable the signal transmission become in the frequency range from $10 \mathrm{kHz}$ to $1 \mathrm{MHz}$, which was also indicated by numerical simulations. Excellent transmission was noticed on thorax, while the extremities and joints lowered the quality of transmission over larger distances. Nie et al. performed in situ characterization of the Human Body Communication (HBC) dynamic propagation channel and investigated the statistical model of the HBC [79]. Measurements were performed using battery-powered equipment (45 MHz carrier signal) in an anechoic RF chamber, on five subjects, and 33 scenarios. They demonstrated that the HBC fading channel is motion-insensitive by means of intensive in vivo experiments and proposed a three-state Fritchman model to describe the burst feature of the HBC channels. Later, using the same equipment, measurements were performed in five real environments for random body motions: in a conference hall, laboratory, grove, playground, and an anechoic chamber [80]. The received power for nine $\mathrm{HBC}$ channels was acquired, and analyzed statistically. HBC demonstrated stable propagation as well as small standard deviation and small range of the received power in all investigated environments. They confirmed that the HBC link is almost insensitive to body motions as well as that the environments tested exerted little influence on HBC propagation channels.

A comprehensive study of the capacitive IBC channel for static and dynamic body positions was performed by Lučev et al. $[10,28,146,152]$. During the in vivo measurements of IBC transmission characteristics for static body positions $[10,146,152]$, different test subjects were asked to maintain a predefined position during the measurement. Meanwhile, the overall geometrical parameters and the return signal path did not change for a specific measurement session. During the measurements of the IBC transmission characteristics for dynamic body positions [28, 146], the subjects under test were asked to move according to the predefined protocol, thus changing the overall system geometry, which affected the return signal path. Preliminary measurements [10] undertaken in an anechoic chamber on four persons in a single still position for five transmitter-receiver distances $(4,16$, 28,36 , and $120 \mathrm{~cm}$ ) were expanded in [146], where measurements on 11 test subjects were performed for four body positions and three types of movements. Despite variations in body proportions and anatomical structures, the results obtained on different subjects for the same test condition were comparable to each other and differed less than $1.5 \mathrm{~dB}$ up to around $10 \mathrm{MHz}$ [146]. In [28] the results were further quantitatively examined and it was shown that up to $10 \mathrm{MHz}$ variations in the gain among different test subjects and the variations caused by different body positions and movements are comparable and change the gain up to around $5 \mathrm{~dB}$ for a given transmitter-receiver distance. For frequencies around $40 \mathrm{MHz}$ this change increases up to around $20 \mathrm{~dB}$, mainly influenced by the body geometry. Finally, the same findings were confirmed in time-domain measurements on 10 test subjects [152].

Limb joint effects on IBC communication were extensively studied by Seyedi et al. [27, 151, 168]. In [27, 168], the effects of the joint presence and joint angle $\left(45^{\circ}, 90^{\circ}, 135^{\circ}\right.$, and $180^{\circ}$ ) on the IBC were studied. It was concluded that the increase in attenuation was proportional to the angle between the forearm and upper arm and the minimum attenuation occurred in the range $80-100 \mathrm{MHz}$ for capacitive coupling. When the joint angle changed from $45^{\circ}$ to $180^{\circ}$ the maximum attenuation difference was $4.2 \mathrm{~dB}$ and $4.7 \mathrm{~dB}$ in the capacitive coupling and galvanic coupling methods, respectively. As expected, capacitive coupling was more sensitive to limb joint position, but galvanic coupling was more dependent on body composition (intrasubject variability). In [151], the study was extended to include an elbow and knee joint, at $20 \mathrm{~cm}$ transmitter-receiver distance, and similar conclusions were drawn. For the capacitive coupling, the minimum attenuation was observed at around $57.4 \mathrm{MHz}$ and the differences between joint and no-joint conditions at this frequency were $2.1 \mathrm{~dB}$ and $1.5 \mathrm{~dB}$ for the elbow and knee joints, respectively. For the galvanic coupling, the minimum attenuation was found at $40-51 \mathrm{MHz}$ frequency range, at which the presence of joints led to around $2.0 \mathrm{~dB}$ higher propagation loss compared to that without joints in the signal path. In summary, it was shown that capacitive coupling is more susceptible to body movement (particularly in the 
upper limbs) at frequencies less than $50 \mathrm{MHz}$, but less susceptible when deployed on the lower limbs. Above this frequency, signal attenuation is more influenced by the environment and it is less dependent on human body composition, indicating that the signal is less coupled through the body.

Influence of body positions and movements on both galvanic and capacitive IBC was also investigated by Callejón et al. in [29]. Galvanic coupling analysis was performed using a signal generator, an oscilloscope, and a pair of balun transformers, in the frequency range from $10 \mathrm{kHz}$ to $2 \mathrm{MHz}$. The galvanic coupling results obtained for three different positions of the same subject (sitting, standing, and walking) were approximately identical, highlighting that neither the position nor the movements of the subject have much influence on IBC galvanic coupling performance; the distance between the floating ground electrodes and the floor was carefully monitored and set between $15 \mathrm{~cm}$ and $135 \mathrm{~cm}$. The measured attenuation was better when the subject was sitting compared to standing or walking, due to the difference in distance between the ground electrode and the external ground plane: the lower this distance is, the better the signal values are. Measurements of galvanic coupling IBC channel on 7 subjects were performed by Gao et al. in $[169,170]$. It was shown that the thicker forearms had lower attenuation, which indicates that the main path of the IBC signals was muscle. The most stable results were obtained in the $20-50 \mathrm{kHz}$ frequency band.

Dependence of signal propagation loss on several poses in capacitive IBC was analyzed by Yokota et al. in [171]. Proprietary hardware based on electrical-to-optical probe [56] and 6.75 MHz frequency signals were used for testing several body positions and poses (raising an arm, extending arms forward, opening the arms, and standing on one leg). The signal losses on the torso were within $\pm 2 \mathrm{~dB}$, but the difference between signal loss on the sides and fronts of the arms was approximately $9 \mathrm{~dB}$. Similarly, gain fluctuations due to the different poses were $4 \mathrm{~dB}$ or less, except for the extending hands forward position $(13 \mathrm{~dB}$ for the down-link and $16 \mathrm{~dB}$ for the up-link). Consequently, they concluded that the best positions for the wearable TX are around the navel or in pants pockets, and wearing them on the arms should be avoided.

As an alternative for measuring influence of body positions and movements on the capacitive IBC, Kazim et al. developed full-wave electromagnetic (EM) models which realistically analyze capacitive body channel communication (BCC), that is, the interaction of capacitive coupler, the human body, and the environment all together [172]. Models were validated with the measurement results of the Philips group [65] and evaluated for numerical uncertainties (boundary conditions, mesh cells) and human body variation uncertainties $(12 \%$ for dielectric properties and $17 \%$ for thicknesses of skin, fat, muscle, and bone layers of the body model) independently. The propagation loss for twenty different body positions in the midfrequency range of $1 \mathrm{MHz}$ to $60 \mathrm{MHz}$ with communication distances of $53,102,116,135$, and $155 \mathrm{~cm}$ was simulated. The simulation results showed that the vertical coupler configuration (one electrode on the skin, another in the air) is less susceptible to physiological variations of underlying tissues compared to the horizontal coupler configuration (both electrodes on the skin). It was shown that the propagation loss in the arm-torso-arm region is the lowest when arms are not touching the torso region irrespective of the distance. Developed methodology has been used in [173] to estimate the two-port complex path impedance matrix $[Z]$ of a capacitive BCC channel in 10 body positions and calculate input and output impedances of a channel, which proved to be capacitive in nature. Therefore, the resistive matching below $1000 \Omega$ and inductive matching between $0.5 \mu \mathrm{H}$ and $5 \mu \mathrm{H}$ either on the transmitter or on the receiver side is sufficient for maximum power transfer in a capacitive IBC system.

The experiments to evaluate the effect of human limb gestures on galvanic coupling IBC channel were carried by Chen et al. in [174]. IBC channels included upper arm, thigh, elbow joint, and knee joint channels. For the upper extremity channels, different elbow joint angles (i.e., $180^{\circ}, 135^{\circ}, 90^{\circ}$, and $45^{\circ}$ ) and hand conditions (i.e., empty-handed, loading with dumbbell, and gripping a force transducer) were considered. For the lower extremity, different knee joint angles (i.e., $180^{\circ}$, $135^{\circ}$, and $90^{\circ}$ ) were evaluated. The experiment results show that channel gain is significantly influenced by the joint angle (i.e., gain variation $1.09-11.70 \mathrm{~dB}, p<0.014$ ). The extension of the channel, as well as the appearance of joint in IBC channel, increases the channel attenuation. The hand loading, gripping force, and muscle fatigue have negligible effect (gain variation $<0.77 \mathrm{~dB} ; p>0.793$ ) on human limb IBC channel. Moreover, the change of joint angle on human limb IBC channel causes significant variation in bit error rate (BER) performance. This is explained by the fact that, in galvanic coupling IBC channel, the majority of electric current is conveyed by muscle tissue (larger than $90 \%$ for frequency lower than $1 \mathrm{MHz}$ ). When the elbow joint flexes (angle decrease), the muscle performs the concentric contraction, and the length of muscle, as well as the channel length, is shortened significantly, resulting in lower channel attenuation. For hand loading or gripping force, the muscle performs the isometric contraction, and the reduction of muscle length is negligible, so that is the change of the channel attenuation. The contribution of the ground loop through the floor in a capacitive IBC system was evaluated by Sasaki et al. in [175]. The received signal strength was measured for two cases: two subjects shaking hands and a subject touching an off-body receiver placed on a stand. Each of the subjects wore a battery-powered transmitter or a receiver on their wrist, and a $10.7 \mathrm{MHz}$ signal was generated. The measured variation of the signal attenuation was approximately $40 \mathrm{~dB}$ depending on which hand the subject used to shake hands or to touch the offbody receiver, while the variation caused by the different floor types (carpet-covered metal floor, concrete floor, hardwood floor, and wooden chair to be above the floor) was less than $5 \mathrm{~dB}$.

In summary, since in the galvanic coupling the signal is confined to the human body, for the same measuring setup the communication is mainly influenced by the anthropometrical and bioelectric properties of a subject, that is, intersubject variability [29, 151, 170]. For example, for IBC units placed on the arm these characteristics are arm 
diameter, tissue thicknesses (such as skin, fat, and muscle), tissue anisotropy, presence of joints in the communication channel, and so on $[29,38,93,174]$. In the capacitive coupling approach, body positions and movements of the subject, as well as change of the environment, can lead to different measurement results for the same setup, while the influence of the anthropometrical characteristics on the transmission are almost negligible [28, 29, 38, 146, 151]. This is because in the capacitive intrabody approach the signal transmission path is closed through the human body, and the return signal path is closed capacitively through the environment $[29,176]$. The return signal path is always the path with the lowest impedance between the receiver and transmitter electrodes. It can be closed through an environment, especially if there are metal objects in an immediate vicinity of the IBC system $[20,146]$, or it can be closed directly from the receiver to the transmitter if they are in line of sight, near each other. For a fixed position of the transmitter and receiver on the body, the signal transmission path is of constant length, regardless of the actual body position. However, if the body position or the environment conditions change, the capacitive return path can change as well [79, 89]: this means that the body can partially block the capacitive return path, which decreases the channel gain [20]. One of the proposed techniques for compensating a change of the return signal path due to the change of body posture was presented in [89]: by estimating current distance between transmitter and receiver ground electrodes, a capacitance between them is estimated and a digitally controlled tunable inductor is employed to dynamically compensate the signal path change, in order to reduce the capacitive coupling loss. Influence of a different environment was reported for higher transmitterreceiver distances: it was negligible for $20 \mathrm{~cm}$ distance in electronic laboratory, classroom, and rugby field [151], and for distances up to $36 \mathrm{~cm}$ in a laboratory and an anechoic chamber [146], but at $120 \mathrm{~cm}$ the gain was up to $10 \mathrm{~dB}$ higher in a laboratory than in the anechoic chamber [146], because the return signal path was coupled through the objects in the vicinity. The distance from electrodes to ground/floor was proved to be an influential factor in $[29,151,175]$ : for lower distances, measured gain was up to $5 \mathrm{~dB}$ higher. This was shown in measurements for sitting versus standing positions [29], signal path through the knee versus through an elbow [151], and standing on a floor versus standing on a wooden chair [175]. Also, in the measurements of the capacitive IBC one of the main influential environmental factors is a stray capacitance $C_{B}$ between the human body and the environment ground [177], which acts as a source of a common voltage coupled into the system. Stray capacitance had also been pointed out by different authors as a possible source of error in tetrapolar bioimpedance measurements above $100 \mathrm{kHz}[178,179]$. By considering only the capacitive coupling from the body segment between potential electrodes to ground, and assuming common values for electrode and body impedances, the authors in [178] obtained a simplified circuit model that predicts gain and nonlinearity errors at any frequency band and resonance at frequencies above $10 \mathrm{MHz}$. They observed a frequency-dependent gain error that increased with increasing frequency $[178,180]$.
3.4. Sources of Artifacts and Noise in IBC. In galvanic coupling the signal path is formed within the human body and the influence of external interfering signals is negligible [96, 181]. However, since in capacitive coupling the environment is an integral part of the signal path, capacitive IBC signal is highly susceptible to external interference such as from power lines $(50 \mathrm{~Hz})$ and other nearby devices $[96,181]$. The main cause of the interference signal in an IBC channel is the body antenna effect $[98,107,182]$ : the human body exposed to an electromagnetic (EM) field behaves as an antenna with its resonance frequency determined by the wavelength equal to twice the human height. In case that the body is grounded, the resonance wavelength is equal to four times that of the height [98]. The body resonance may occur at different frequencies, depending on various environmental conditions, so the human body operates as a wideband antenna in $40-400 \mathrm{MHz}$ frequency range [98]. The IBC channel is especially affected by interferences below $1 \mathrm{MHz}$, while for higher frequencies the observed interference level is below $-75 \mathrm{dBm}$ [107]. However, in the frequency modulation (FM) band $(80-110 \mathrm{MHz})$ this level may rise to $-30 \mathrm{dBm}$ $[98,107]$.

Schenk et al. performed interference characterization measurements at different locations in a laboratory using a battery-powered spectrum analyzer [65]. The interference was almost negligible for a subject located in the middle of a room. However, as the subject approached a monitor, a measuring equipment, or a table with metal base, the interference was considerably increased. This was explained by the fact that nearby devices and furniture act as an antenna for sources of interference and in turn couple this to the electrodes/body. Xu et al. studied the influence of an environment and interference from nearby capacitive IBC devices, as well as overall interference over air channel [20]. They used proprietary battery-powered IBC devices with signal electrodes connected to a test subject's wrist (120 cm distance) in the $20-100 \mathrm{MHz}$ frequency range. The influence of the environment was studied with a wall, another person, and an external copper plane close to the test subject. Interference from IBC devices worn by another person or placed on a nearby table were tested for several body positions, and interference coupled through air was studied using only the receiver end, with the transmitter turned off. Generally, the environment does not affect the transmission unless the objects are closely coupled to the transmitter or receiver ground planes. With a closely coupled conductive object, whether grounded or floating, the capacitive channel gain will increase. On the other hand, the strongest measured interference from the capacitive IBC devices placed $80 \mathrm{~cm}$ away was only $8 \mathrm{~dB}$ smaller than the received signal in the current IBC channel. The air-channel interference inside and outside is generally lower than $-120 \mathrm{dBm} / \mathrm{Hz}$ in the frequency range from 35 to $88 \mathrm{MHz}$. Within this band, the capacitive IBC channel interference was mainly coupled from the receiver board. At higher frequencies, the interference was mainly from the FM radio signal and at lower frequencies from indoor electronics. Effect of electromagnetic interference (EMI) on IBC communication was studied by Hwang et al. in [182]. The human subject held the receiver module 
while touching the electrode during exposure to a general EMI environment at a total of 100 measurement sites in both indoor (office, commercial building, and train/subway station) and outdoor environments. Analysis results showed that the interference signal received at the receiver module was primarily caused by the man-made noise sources. This corruption leads to severe bit-error-rate (BER) degradation of the IBC device. Based on the measurements, the interference model was developed and used in the design of an interference rejection filter placed just after the receiver electrode.

A comprehensive noise analysis for capacitive IBC was reported by researchers from several Japanese institutions $[13,14,56,133,180,183-185]$. They showed that IBC systems are affected by large common-mode noise from various kinds of electronic equipment in living spaces, such as lighting devices, air conditioners, liquid crystal televisions, and refrigerators $[13,14]$. In their measurements they used a battery-powered wearable transmitter generating $6.75 \mathrm{MHz}$ frequency and an AC-driven embedded receiver, which was shown to be very prone to the environmental noise (noise power spectrum with $\mathrm{AC}$ power is more than $20 \mathrm{~dB}$ larger than that with battery power). A capacitance model of such a system was developed in [185]. Kado et al. showed that packet error rates (PERs) can be reduced by implementing a common-mode choke coil to the embedded receiver [56]. Due to the noise, a parasitic impedance is added to the transmission line, which results in degradation of the transmission line's impedance balance between the signal and the ground line. As the transmission line becomes longer, the differential-mode noise damps, so just a common-mode noise between the earth and the floor ground was recognized as the main noise factor in an IBC system. An effective way of improving impedance balance and reducing common-mode noise in the system was connecting an additional electrode or a compact capacitor to a ground electrode, according to $[13,14]$ (Figure 13). Two types of noise, excluding transceivers noise, are coupled to the embedded receiver [183]: radiated noise via human body $[133,183,184]$ and conducted noise via floor ground [180]. Noisy-channel models of a capacitive IBC system with an embedded receiver situated in the vicinity of a wall was investigated by Sasaki et al. in [180]. In a model based on a Wheatstone bridge a virtual voltage noise source, assumed to exist between the wall and floor, emulates the effects of the noise transmitted through the grounding systems (conducted noise). It is found from the model that the noise is suppressed when the Wheatstone bridge is balanced, which can be realized by tuning a variable capacitor in a developed noise-reduction mechanism (NRM) consisting of an auxiliary electrode and a variable capacitor. It was observed that, by tuning the variable capacitor with the NRM, the noise voltages were successfully reduced, while the signal voltages were almost unchanged. As a result, the SNR was increased. Another noise component, radiated noise, was explained in $[133,184]$ by means of electrostatic analysis based on the method of moments. The proposed circuit model contains a total of 20 parameters, namely, 15 capacitances and 5 equivalent noise sources. It was found that since it is difficult to avoid the radiated noise via the human body, the reduction

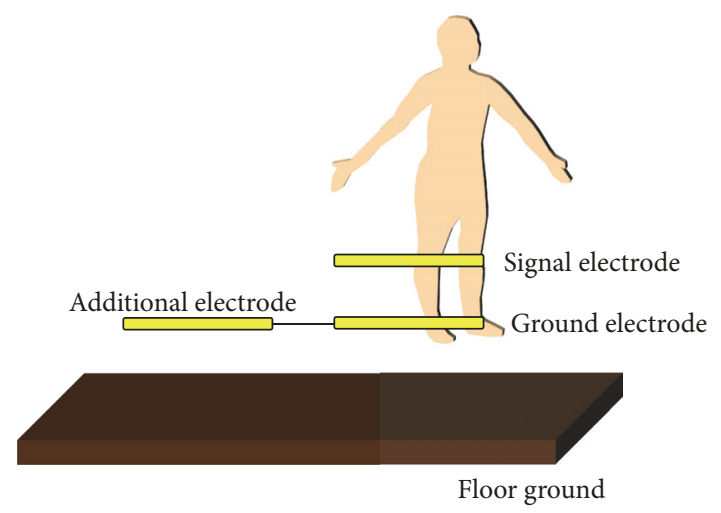

FIGURE 13: Setup for an additional electrode effect. Connecting an additional electrode $(365 \mathrm{~mm} \times 500 \mathrm{~mm} \times 0.3 \mathrm{~mm})$ to a ground electrode causes a common-mode noise reduction and improves the impedance balance $[13,14]$.

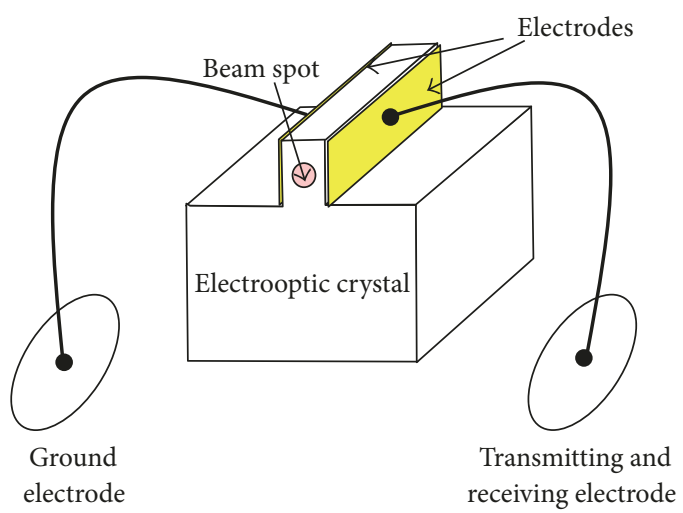

FIGURE 14: Schematic of an electrooptical sensor. Received signal modulates polarization of the laser beam passing through the crystal, thus reducing the noise and eliminating the influence of the floating ground potential [15].

of noise sources in intrabody communication is mandatory. One of the proposed ways to reduce the common-mode noise measured at the IBC receiver is using an electrical-tooptical (EO) sensor for picking up the received signal [15, 73] (Figure 14). Thanks to the sensor's extremely high input impedance, the measured electrical noise can be reduced. Moreover, the ground electrode of the EO sensor is electrically isolated from the electronic circuits, thus eliminating the influence of the floating ground potential. As a result, both the noise and the distortion of the receiving signal can be greatly decreased [73].

\section{IBC Transceivers and Devices}

Three fundamental stages of any communication system are transmitter, communication channel, and receiver $[176,186]$. A typical IBC transmitter consists of a sensor, an analog-todigital converter (ADC) for analog preprocessing, a modulator, and a coupler with electrodes, adapted to the type of the IBC technique used. Choice of sensors depends on a desired 


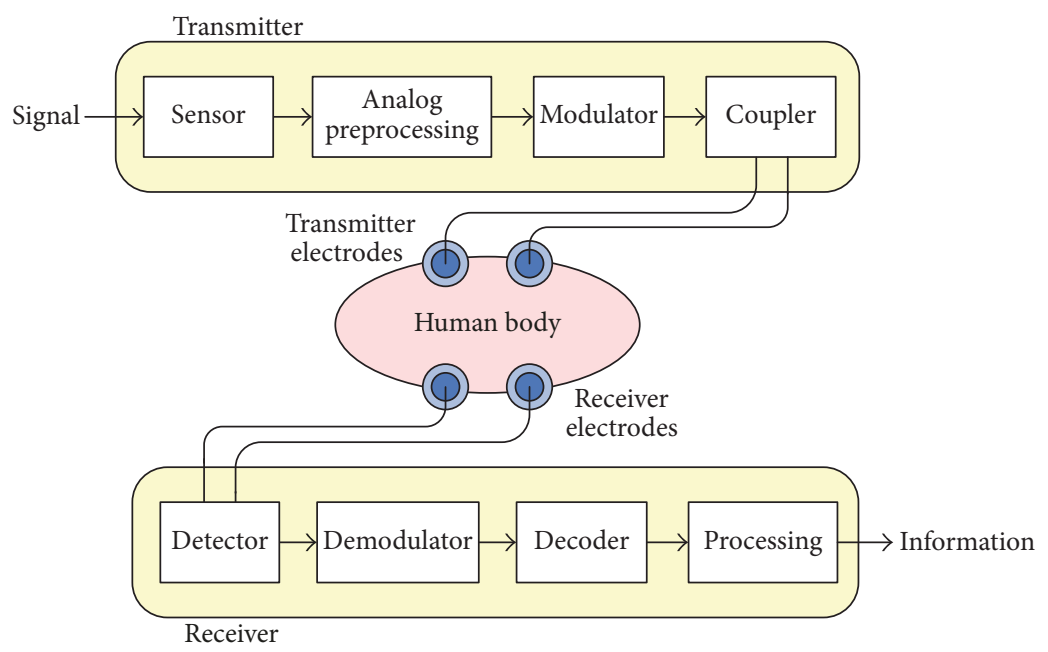

FIGURE 15: Intrabody communication system block diagram. Its main parts are transmitter, communication channel, and receiver.

application, that is, sensors of physiological functions for obtaining biomedical signals (like EMG, ECG, and EEG) in medical applications, or cameras and microphones for media devices. A communication channel refers to a physical transmission path between the transmitter and receiver electrodes and comprises a human body and a surrounding environment. Transmitter and receiver electrodes can be connected to the body but can also be left floating, depending on the signal frequency, coupling technique, and application [146, 187, 188] (see Section 3.1). A typical IBC receiver consists of a detector with electrodes, demodulator, signal decoder, and a signal processing unit which provides desired information. Main components of an intrabody communication system are shown in Figure 15. In some cases, transmitter and receiver units can be embedded in a single IBC transceiver with a common control unit. There is no definite principle for the electronic design of the IBC transceiver units. They should be small and light and have full integration ability and energy efficiency and have the ability to transmit at low power. Therefore, main parameters that need to be considered during the IBC transceiver design are transmitter output power, receiver sensitivity (minimum signal power required to receive data correctly), carrier signal frequency, data rate, modulation method, and communication interface (transmitter output and receiver input comprising both couplers and electrodes) $[176,186]$. Transmitter output power should be kept as low as possible due to the health and safety reasons (see Section 2.3) and battery longevity issues, yet high enough in order for signal to be detected by the receiver placed at the desired distance from the transmitter. Carrier signal frequency, data rate, modulation method, and communication interface all depend on the choice of the coupling technique and application.

A general overview and comparison of the design and implementation of IBC transceivers is given in Section 4.1, separately for the IBC systems developed using discrete components (Section 4.1.1) and in CMOS technology (Section 4.1.2). A discussion on modulation techniques and power consumption is presented in Section 4.2.
4.1. Design and Implementation of IBC Transceivers. Several groups from all parts of the world are involved in the development of IBC devices. Various developed IBC systems can be found in the literature, some of which are more oriented to medical applications, while the others are oriented to various nonmedical purposes. The developed systems differ by the coupling method, the coupling amplitude, the chosen frequency range, the signal modulation method, and the achieved data rates $[176,186]$.

4.1.1. IBC Systems Developed Using Discrete Components. In the early days of IBC development, the researchers were mostly concentrated on developing proprietary IBC devices and testing their characteristics and functionality $[5,7,32,44$, 53, 68, 69, 90, 91, 93, 94, 189]. The concept of Personal Area Networks (PANs) was presented in 1995 by Zimmerman to demonstrate how electronic devices on and near the human body can exchange digital information through near-field electrostatic coupling [7]. Independently of Zimmerman, two galvanic IBC systems were developed: wireless system with very low consumption designed for monitoring the ECG signal by Handa et al. [90] in 1997 and a system for communication with implantable transducers by Lindsey et al. in 1998 [91]. A group from NTT Human Interface Laboratories was focused on connecting electronic devices in everyday life by a simple touch $[15,55,57]$ and developed an indoor wireless-like networking and positioning system for connecting portable and wearable devices (home and office appliances) to the network while the user stands or walks on the floor [54, 56, 58-60]. In biomedical applications field, IBC was employed in various general biomedical systems $[66,67$, $70,71,85,86,95,96,190]$ or specifically for ECG $[87,93,94]$, EMG $[68,69]$, and human posture $[83,84]$ monitoring, as well as for monitoring and controlling artificial hearts and other artificial organs in the body [77, 78]. IBC systems developed for the characterization of IBC channel and human body as a signal transmission medium $[19,20,33,41,61-$ $65,72-76,79-82,88,89,93,94]$ are mostly built around DDS or FPGA circuits for signal generation at one or in the range 
of frequencies and for the detection of received signal power at the receiver end.

A comparison between several proprietary capacitive and galvanic IBC systems found in the literature and developed using discrete components is given in Tables 5 and 6 , respectively. The institution and first authors of each system, the amplitude and frequency of carrier signals, the modulation method, the maximum achieved data rate, power consumption, and the proposed application are all provided.

4.1.2. IBC Systems Developed in CMOS Technology. In parallel to the IBC systems developed using discrete components, application-specific custom designed integrated circuits (ASIC) transceivers implemented in CMOS technology have also been developed by several research groups. A pioneering work on several generations of CMOS-based IBC systems has been performed by a large research group from KAIST (Korea Advanced Institute of Science and Technology). An overview of the IBC transceivers evolution implemented with CMOS technology by the KAIST group can be found in [102, 191]. A first HBC transceiver [97], based on a wideband signaling (WBS) technique with a directcoupled interface (DCI), was developed in 2006 and used in a prototype wearable audio MP3 player system to play back the digital audio signal transmitted through the wearer's body without any wire [192]. An improved empirical channel model of the human body [35] was used in the design of a transceiver which supports communication between one transmitter and a number of receivers in a network [193], and the body antenna effect (signal distortion due to the cellular or radio signal absorbed by the body) has been taken into account in IBC design since 2008 [98]. Later, a dualband IBC transceiver integrated with the Medical Implant Communication Service (MICS) circuits, sharing the same front-end, was designed to cover the implantable network communication as well [99]. The transceiver operates in $30-70 \mathrm{MHz}$ band for body channel communication (BCC) and $402-405 \mathrm{MHz}$ band for MICS. Next, more accurate body channel model based on Maxwell's equations [17] was actively used in the design of a high performance energy-efficient transceiver [26, 194]. Based on the channel analysis, the resonance matching and contact impedance sensing (CIS) techniques are proposed to enhance the quality of the body channel. To overcome power and cost issues regarding the use of crystal oscillator, a crystal-less transceiver was implemented [100] by using an injection-locking digitally controlled oscillator (IL-DCO) with autofrequency calibration technique, and a scalable double-FSK modulation scheme with divider-based switching transmitter. In 2013 a worldfirst WBAN transceiver satisfying all of the specifications for IEEE 802.15.6 HBC standard [6] was presented in [101]. Stringent transmitter spectral mask requirement was achieved implementing the driver active-digital-bandpass filter (ADF) without using external components. Addition of a duty cycle control (DCC) to the chip [101] resulted in a new low-power transceiver [102] optimized for the network system operation rather than just the TX circuit operation, like the previous generations of HBC transceivers. It can provide other additional functions including the channel access control with the
MAC (Medium Access Control) scheduler, the encryption with the zero delay cipher (ZDC), and the energy detection with the received signal strength indicator (RSSI). Next generation fully IEEE 802.15.6 HBC compatible transceiver $[103,195]$ was optimized for low power consumption. Most of the described BCC research at KAIST used only the frequency band below $100 \mathrm{MHz}$ and the group was mainly focused on low data rate $(<10 \mathrm{Mb} / \mathrm{s})$ healthcare applications [26, 97-103, 193-195]. In 2015, a possibility of achieving high data rates for multimedia transfer was investigated [104-106] and two kinds of BCC transceivers were implemented in a new single chip: a low-energy $40 / 160 \mathrm{MHz}$ dual-wideband full-duplex BCC transceiver for entertainment applications (ET-mode) and a 13.56 MHz RC oscillator-based superregenerative transceiver for healthcare applications (HC-mode). Also, new body channel measurements corroborated that the body channel bandwidth can be extended up to $200 \mathrm{MHz}$.

CMOS transceivers were also designed by other research groups, like [107-114, 119-122, 196, 197]. Saadeh et al. presented several chips for binaural hearing aids application that mitigate some or all body channel impairments: interference and signal multipath in $[115,116]$, together with variable ground effect and variable skin-electrode impedance in [117, 118]. One of the rare galvanically coupled (GC) IBC receivers was designed by Chen et al. [198]. It is resilient to frequency misalignment for short-distance communication due to the carrier tracking technique. A concept of transmitting IBC signals through a mouse or rat brain was reported and tested in vivo in [199-201], while no effect on normal neural activities was noticed.

Detailed performance specifications of the CMOS-based IBC transceivers are listed in Tables 7 and 8.

4.2. IBC Modulation and Power Consumption. Due to the distinct characteristics of the galvanic and capacitive IBC, different types of modulation and demodulation methods have been utilized in the IBC systems. Zimmerman proposed the first prototype of capacitive coupling IBC in 1995 [7]. Both On-Off Keying (OOK) modulation scheme and direct sequence spread spectrum (DSSS) were investigated in this preliminary design. Despite the accuracy of the results achieved from DSSS, the OOK scheme was selected due to its simple hardware and implementation. The achieved data rate and power consumption were $2.4 \mathrm{~kb} / \mathrm{s}$ and $333 \mathrm{~mW}$, respectively, in the first OOK-IBC method proposed in [7]. In 2006, Ruiz and Shimamoto [202] evaluated various digital modulations in the IBC system. The achieved results from their experiments demonstrated that the most suitable modulation schemes for IBC were binary phaseshift keying (BPSK) as well as minimum shift keying (MSK) [202]. However, all experiments were carried out in the frequency range of $200-600 \mathrm{MHz}$. The wideband signaling (WBS) communication technique was used to implement the capacitive coupled IBC system by Song et al. in 2007 [97]. This method distributes the non-return-to-zero (NRZ) digital data through the body channel without utilizing any signal modulation block. The implementation and fabrication of the transceiver in such a system is of less complexity compared to the methods that are employed in the modulation schemes. 


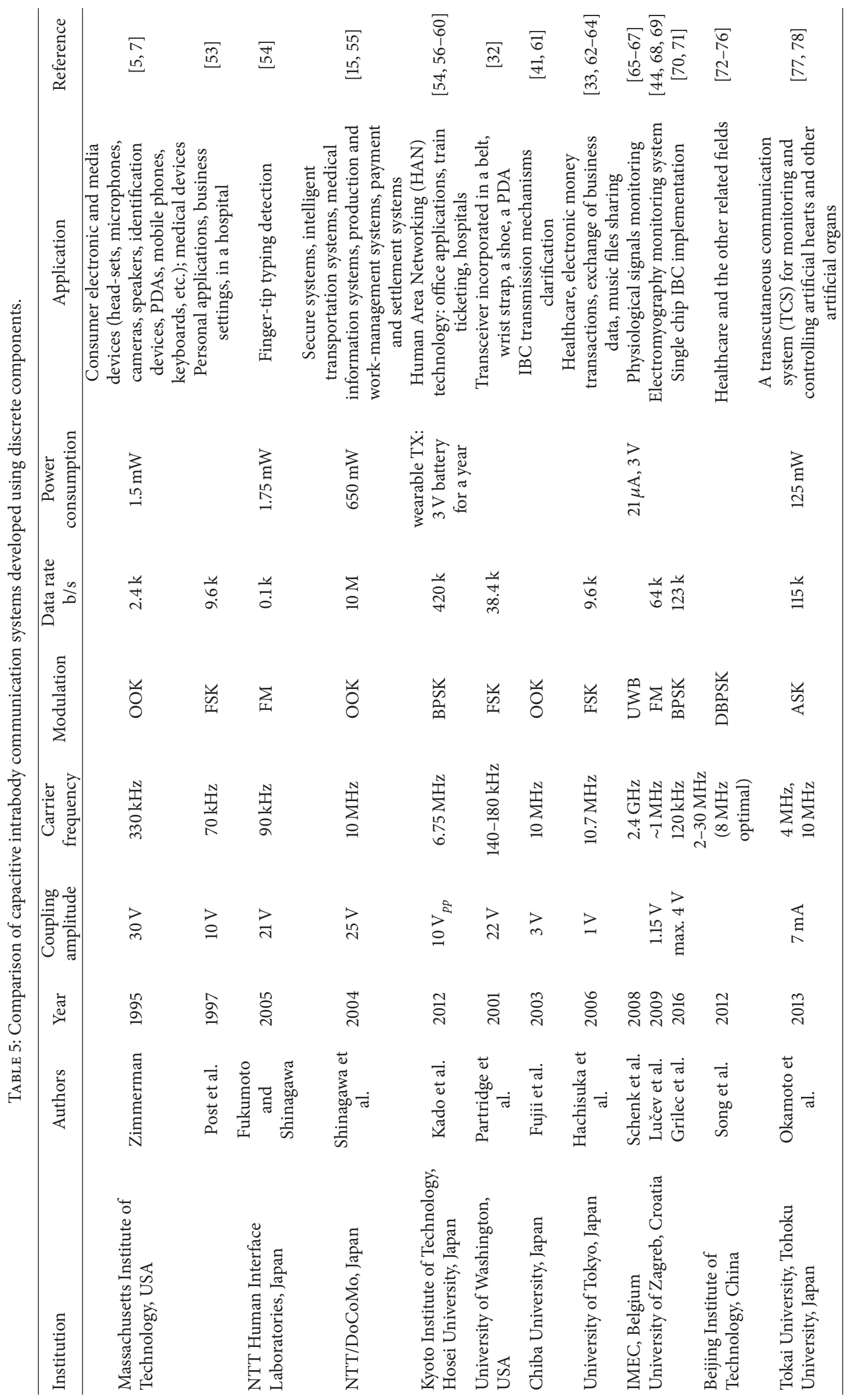



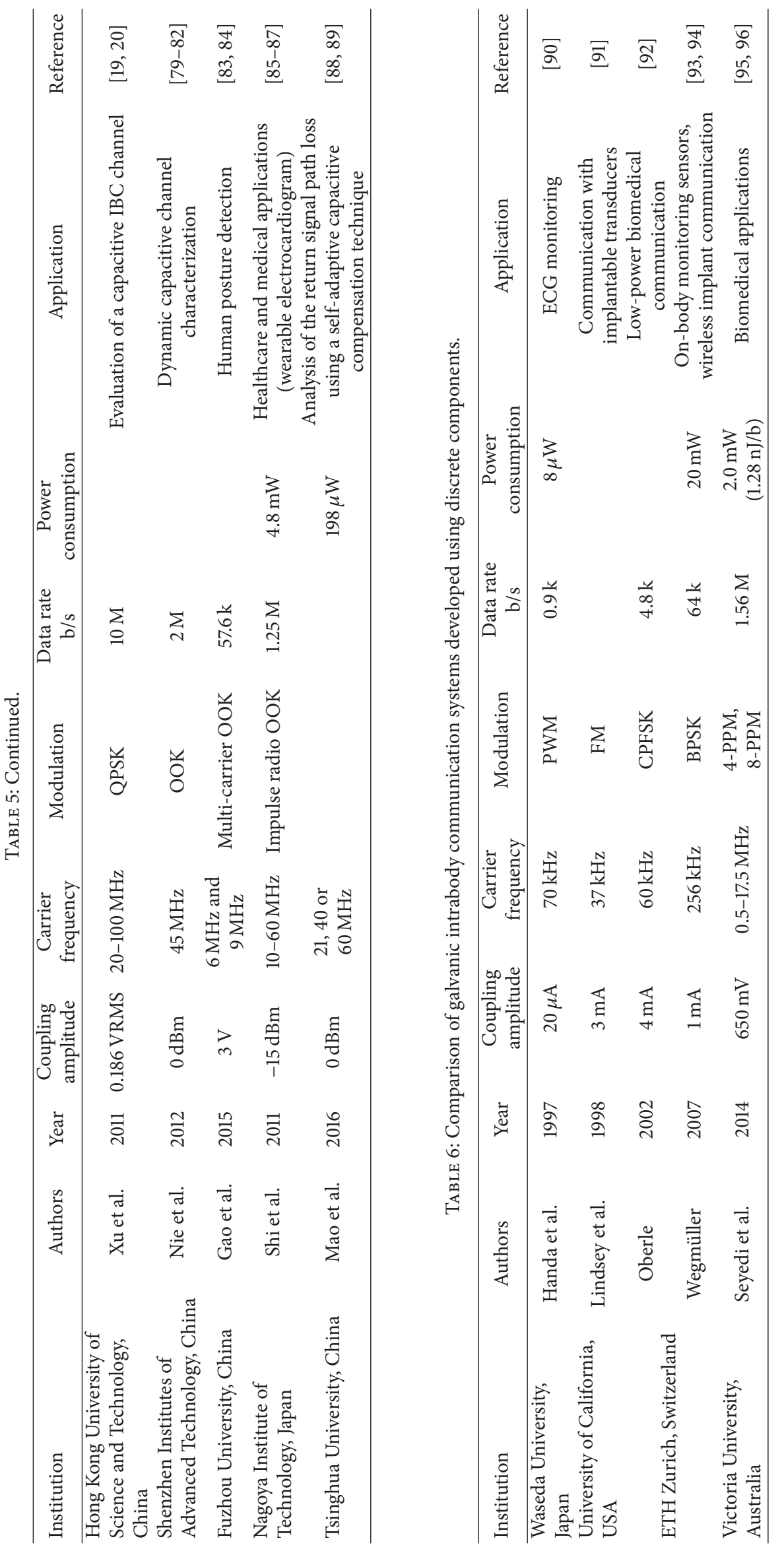


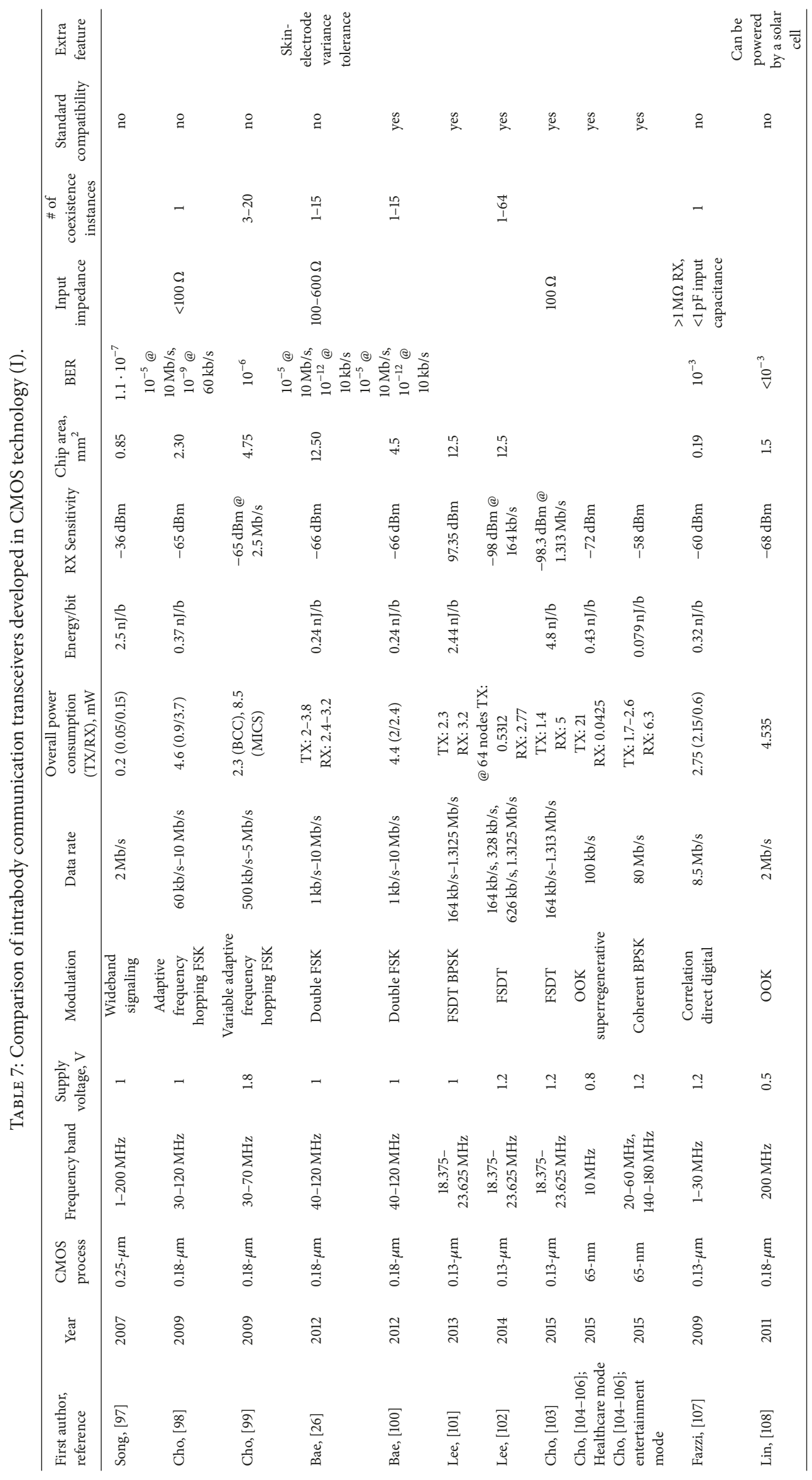




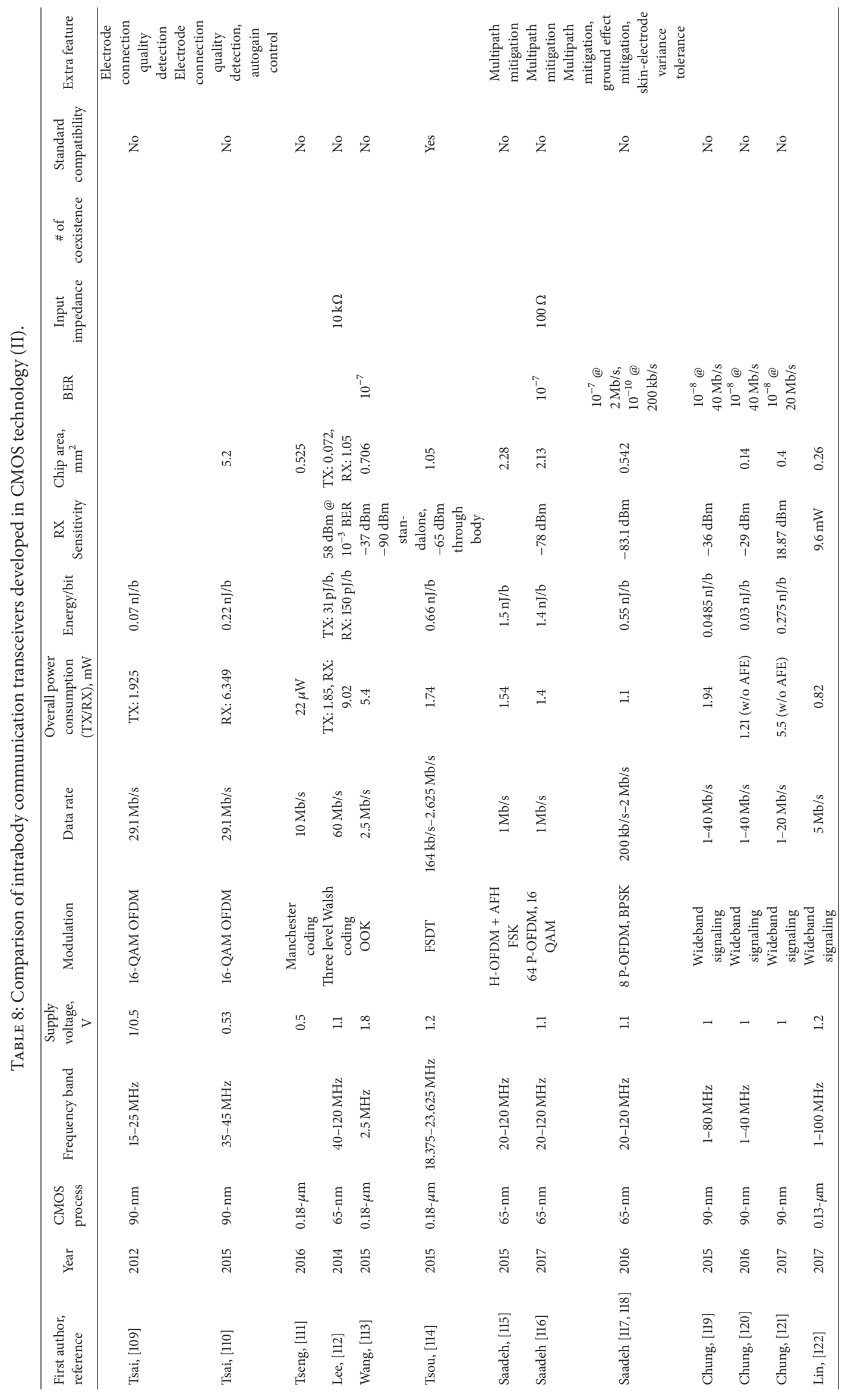


Yet, according to a recent study on the capacitive coupling, the WBS was introduced as the most reliable technique in IBC system [122]. In this study, the proposed WBS-IBC system obtained data rates up to $5 \mathrm{Mb} / \mathrm{s}$, which are the most satisfactory results among the nonmodulation capacitive coupling IBC systems [122]. However, the direct voltage or power transmission through the body might lead to hazard and body damage and it is therefore not suitable for the IBC medical applications. Recently, the Impulse Radio (IR) technique was employed to develop an electrocardiogram (ECG) with capacitive coupling IBC [87]. In the IR technique, short pulses using either OOK or pulse position modulation (PPM) scheme are propagated through the communication channel. The possibility of designing a low power consumption, high data rate, and short-distance communication system is fulfilled while using the IR technique. Wang et al. [87] used an OOK modulation scheme for designing a capacitive coupling IBC transmitter and receiver for the ECG applications. The system performance showed a data rate of $1.25 \mathrm{Mb} / \mathrm{s}$ as well as low power consumption of $4.8 \mathrm{~mW}$. The modulation scheme used in the first implemented galvanic coupling IBC was the continuous phase frequency shift keying (CPFSK) by Oberle in 2002 [92]. The CPFSK is a type of traditional FSK which is commonly utilized in the data propagation systems with low data rate. An extensive study was carried out by Wegmueller et al. on the galvanic coupling IBC between 2005 and 2010 [24, 34, 203]. During their IBC transceiver design, both FSK and BPSK were used as digital modulation schemes. By employing the BPSK the achieved data rate reached $64 \mathrm{~kb} / \mathrm{s}$ which was higher compared to the first designed galvanic IBC, that is, $4.8 \mathrm{~kb} / \mathrm{s}$. However, the overall power consumption of the proposed IBC system was $726 \mathrm{~mW}$. Chen et al. [204] investigated the performance of the three different modulation schemes including BPSK, QPSK, and 8PSK during the galvanic coupling IBC. The authors focused on the BER performance while the carrier frequency of transmitted signal was $500 \mathrm{kHz}$. The achieved results from both theoretical and simulation results indicated desirable performance for QPSK compared to BPSK or 8PSK. The lowest BER with higher bandwidth efficiency and data rate was obtained by QPSK within the body channel length of $11 \mathrm{~cm}$. The satisfactory results achieved through the IR technique led Seyedi et al. [96] to employ the IR-PPM scheme in order to design the galvanic coupling IBC transmitter. The propagated baseband signal through the human body channel was modulated using PPM. The achieved results indicate a higher energy efficiency around $1.28 \mathrm{~nJ} / \mathrm{b}$ compared to Wegmueller's proposed transmitter, that is, $457 \mathrm{~nJ} / \mathrm{b}$. Recently, the body channel characteristics such as path loss were examined by employing both 4- and 8-PPM schemes [95]. The assessment of both bit error rate (BER) and SNR shows better outcome for the 8-PPM scheme compared to 4-PPM and it is a suitable choice for the galvanic IBC.

\section{IBC Applications and Systems}

In this section an overview of IBC applications and systems is presented. The first part of the section shows some practical

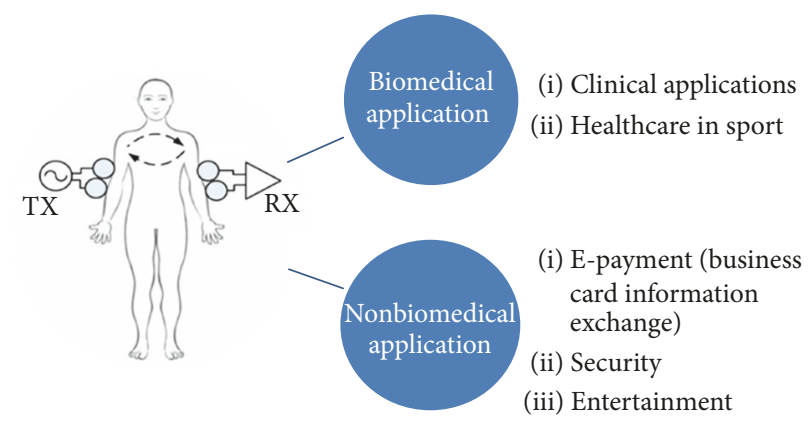

FIGURE 16: IBC applications.

examples of IBC applications in medicine and other fields. The second part of the section analyzes the use of IBC for communication with implants. Finally, the third part of the section shows new approaches in IBC related to nanotechnologies.

5.1. IBC in Medicine, Systems, and Applications. Generally, the IBC system applications could be divided into two main categories: biomedical applications and nonbiomedical applications (see Figure 16). The IBC biomedical applications are used to communicate healthcare information among subjects and clinical experts. Yet, nonbiomedical applications of IBC are employed for information communications without clinical purposes. The initial IBC proposed by Zimmerman was used for a nonbiomedical application when he designed a personal area network (PAN) prototype [7]. The prototype was used to exchange the electronic business card information by shaking hands by means of the proposed IBC system. In the PAN, the wireline communication was replaced by the human tissues and the signals were propagated through the body itself.

Based on the positions of IBC devices on the body, intrabody communication systems can be divided into two large groups: (1) at least two IBC devices are constantly on, in, or near the same user and (2) one IBC device is worn by the user, and the other is on a different user or embedded in an environment. First group of possible applications include, but is not limited to, continuous physiological signal monitoring (IBC devices can be on or implanted in the human body) or continuous data transmission (like data, audio, and video streaming). In the second group the communication is established when the user touches or comes very close to the other IBC device, as in data exchange between two users during the handshake or user identification at the IBC gateway.

The features of IBC such as being interference-free, energy efficiency, antenna-less transmission, and high security have introduced IBC as a communications support technology in emerging biomedical applications. Several studies have investigated using the IBC technique in medicine [186]. Hachisuka et al. [63] successfully measured the heart rate as well as a person's oxygen saturation $\left(\mathrm{SpO}_{2}\right)$ using IBC technique. However, their system suffered from the lower data rate, that is, $9.6 \mathrm{~kb} / \mathrm{s}$, compared to the newer IBC systems. In 2009, due to the appropriate results of IBC, 
the galvanic coupling of IBC technology was employed in the wireless implant communications [51]. In this study, Wegmueller et al. carried out the phantom measurements using miniaturized IBC transceiver pills [51]. Although the achieved results verified the performance of the proposed IBC system, the results were obtained from the homogeneous phantom medium rather than the multilayer body issue. Recently, some in vivo experiments were carried out on a pig body to examine the use of IBC technique in biomedical implant applications [82]. In this study, five electrodes were utilized including one implanted transmitter and four surface receiver electrodes. The authors investigated the influence of various electrodes on the channel gain. The channel length varied from 280 to $850 \mathrm{~mm}$. The results indicated that the electrode type effects on the channel gain could be neglected using IBC technique.

Another application of IBC communication technology is the human area networking system called RedTacton developed by Nippon Telegraph and Telephone Corporation (NTT) [205]. This system uses the skin surface to communicate data with the rate up to $10 \mathrm{Mb} / \mathrm{s}$. RedTacton provides highly secure data propagation through the body channel just by shaking the nearby person's hand or touching the electronic devices around the body such as printers. The features of RedTacton could be also led to new trends in employing IBC in daily life such as the security and E-payment applications. Song et al. [192] proposed a novel digital wearable audio player system by applying the capacitive coupling IBC approach. In their system, the audio signal communicates between the audio player and the earset receiver through the user's skin when the user touches the backside single electrode of the audio player. The reported data rate of the transceiver was $2 \mathrm{Mb} / \mathrm{s}$ while the system power consumption was around $5 \mathrm{~mW}$. According to Song et al. [192], not only does the IBC-based audio player system indicate the higher energy efficiency but also the better audio quality is achieved compared with the Bluetooth technique.

In the image-guided brain surgery, the real-time display of a tracked instrument is possible through navigation technology. Nowadays, electromagnetic tracking (EMT) devices are the most common technology used for position tracking [206]. Electromagnetic sensors are utilized in these systems to collect the position of the tracked surgical instrument by cable. The replacement of these sensors by wireless ones could lead to data transmission outside the body using the IBC technique.

A new concept of wireless transmission of neural signals within the brain, called intrabrain communication (IBCOM), was presented by Al-Ashmouny et al. [199]. In this work, two miniaturized IBCOM chips were designed and tested in vivo on a rat's brain, with no effect on normal neural activities. Transmission distance, limited by the size of the rat's brain, was $15 \mathrm{~mm}$. Similar research on wireless image data transmission through a mouse's brain was reported in [200] and called wireless intrabrain communication (WIBCOM) method. Another demonstration of wireless data transmission through a mouse's brain by intrabody communication employed an implantable microsized image sensor [201].
Intrabody nanonetworks bring together the concepts of intrabody communications and nanotechnologies for application in new treatment and diagnostic techniques [207]. Research in this field mainly focuses on the study of the terahertz band as well as the investigation of new nanoantennas based on nanomaterials [208]. The miniaturization of a conventional antenna to meet the size requirements of a nanosensor results in very high resonance frequencies, in the order of several hundred terahertz [209]. One of the applications of intrabody nanonetworks has been the monitoring of the human nervous system. Communication with nanomachines implanted in the living human brain has already been used in the treatment for drug-resistant epilepsy [210]. In [211], a nanoscale stimulator device called synaptic nanomachine $(\mathrm{SnM})$ was presented, which had effects on the synchronization of neurons and their oscillatory behaviors. These effects are the basis of cognitive and behavioral functions, such as brain learning and plasticity. In addition, the results presented in [212] focus on the design of appropriate stimuli to evoke the desired synaptic modifications in terms of strengthening and weakening. On the other hand, the authors of [213] proposed the use of natural neurons as elements of access to biosensors and bioactuators implanted at nanoscale.

5.2. IBC for Implanted Systems. Implanted sensors can be used to continuously measure relevant physiological parameters, paving the way to novel approaches for health monitoring and care delivery [214]. Implanted miniaturized devices could enable efficient healthcare and clinical applications, such as monitoring, diagnosis, and treatment $[76,155]$.

Currently, medical implants use narrow band technology with a carrier frequency at the MICS band between 402 and $405 \mathrm{MHz}$ [215]. Inductive coupling and radio frequency (RF) telemetry are frequently used for implanted communications [216]. However, one of the main drawbacks for achieving an efficient communication link between implanted devices is posed by the fact that human body is primarily composed by water, a medium through which RF electromagnetic waves do not propagate well, even at relatively low frequencies [217]. The design of antennas for medical implants is very challenging due to the small size, low power, biocompatibility [215], and safety requirements [49], among others.

This way, IBC has often been proposed in the literature to overcome some of these issues. Compared with other communication methods, implanted IBC has the main advantage of low transmission power through the use of miniaturized electrodes [75]. Therefore, IBC is less susceptible to eavesdropping and provides higher security to implanted devices [218]. Because of the nonconductive nature of the free space outside the surface of the skin, the signal is confined within the human body making it an inherently secure communication channel [49]. It must be noticed that patient safety must be ensured and induced currents should not cause nerve stimulation and not interfere with body signals in the operating frequency range [24]. Therefore, signals at frequencies below $10 \mathrm{kHz}$ have to be avoided.

Due to the inherent difficulty of implant experimentation, physical models and simulation approaches are common 


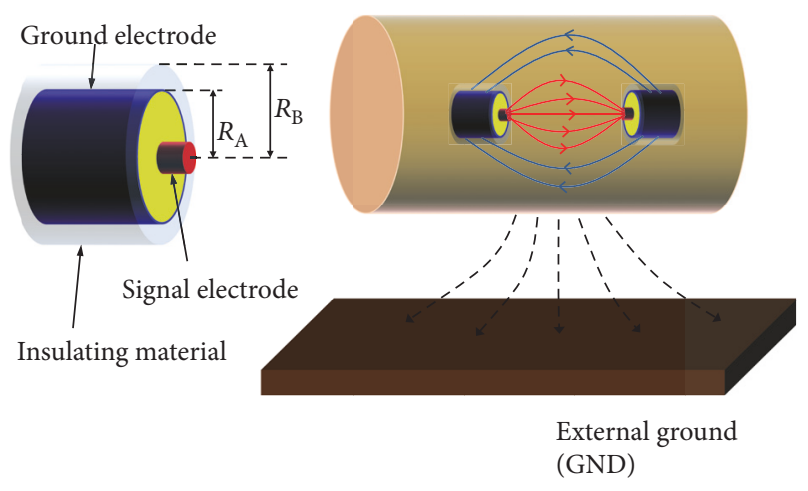

FIGURE 17: Coaxial capacitive electrodes for implantable intrabody communication system.

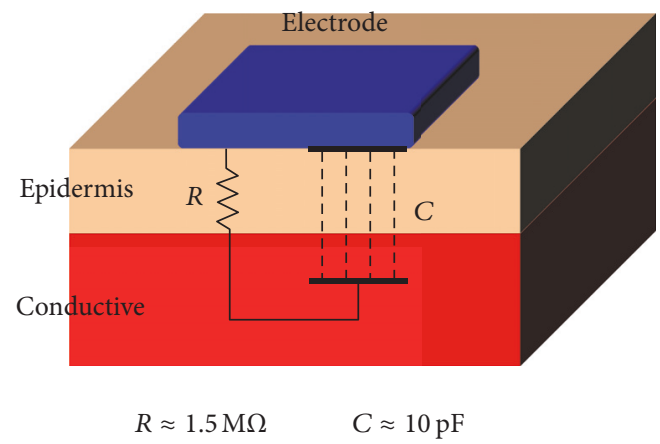

FIGURE 18: Electrical model for the body communication with implants [16]. An electrode forms a capacitive link with the inner tissues of the human body (the electrode is one plate of a parallel plate capacitor, with the conductive tissues form the other plate).

methods in research studies. Wegmueller et al. [34] used MSL27 liquid in their experiments to emulate muscle tissue and securely characterize implanted IBC transmission in the thorax. A galvanic transmission system for a pacemaker and an implanted pill were evaluated in a series of in vitro experiments according to this method [51]. Simulation results for implanted sensors at $60-\mathrm{mm}$ deep from the body surface predicted $35 \mathrm{~dB}$ and $50 \mathrm{~dB}$ signal attenuation for galvanic coupling and RF techniques, respectively [186].

The floating electrode in capacitive coupling makes its implementation in implantable devices challenging [174]. Li et al. [76] proposed an implantable intrabody communication system based on capacitive coupling (see Figure 17). They found that the signal attenuation decreased as the interelectrode distance increased from 5 to $9 \mathrm{~cm}$ [82]. It has also been reported that the signal attenuation decreases with the increase of the height between the body and the ground up to a certain value from which it remains approximately constant [76]. Another approach is the one proposed by Anderson and Sodini in [16] (see Figure 18), integrating the internal conductive tissues of the body as active elements in the capacitive coupling. This technique was experimentally validated in the frequency range between $10 \mathrm{MHz}$ and $150 \mathrm{MHz}$.
A major problem when dealing with implants is the possibility of rejection. This issue was analyzed by Okamoto et al. considering two different implanted electrodes such as platiniridium (diameter of $0.3 \mathrm{~mm}$ and length of $15 \mathrm{~mm}$ ) and titanium mesh electrodes [77]. The titanium mesh electrodes showed improved transmission performance together with excellent histocompatibility properties [219]. Energy efficiency has also been analyzed in the context of IBC implants. Sun et al. [220] implemented a surface implant transmitter with $\mathrm{x}$-shaped electrodes to improve current flow, thus reducing energy consumption. The transmission performance in a multihop scenario can be enhanced if the implants are placed in the boundary of different tissue layers exhibiting different reflection coefficients [221]. The power consumption has been reduced to only 10.8 - $\mathrm{mW}$ for a medical implant transceiver, as it was reported in [20].

IBC implanted systems have been applied to ECG monitoring [90], wireless intrabrain communication [186], or monitoring of artificial organs inside the body [78]; however, its expectation of use is increasing due to the numerous advantages that this communication method offers. Moreover, the recent advances in micro-nano-technology are enabling a new generation of nanoscale implantable devices inside the human body $[222,223]$ to detect specific molecules in vivo, and IBC technique can play an important role in this regard.

5.3. Nanoscale Intrabody Communication. Most of the successful medical devices for physiological signs detection (e.g., blood pressure, photoplethysmogram, electrocardiography, and electromyography) are placed on the body surface or near it [223]. With the rapid development of nanotechnology, such as graphene or metamaterials, the implementation of nanoscale implantable sensors for the specific molecules in vivo became possible [224]. The nanosensors may be implanted into the organs, detecting specific symptoms or virus and forwarding the sensing data to the router node in body [225]. However, so far, researchers have paid more attention to the biophysical or biochemical abilities of these implantable nanosensors. Communication techniques are urgently needed inside the body for these nanosensors to enable the coordinated sensing and actuation of biochemical implants, to extract information about deep tissues and cells, and to export it through an external gateway out the body for further processing.

5.3.1. Intrabody Molecular Communication. Several communication paradigms are considered for intrabody communications in nanoscale. The most promising one is the intrabody molecular communications (MC), which employs molecules to encode, transmit, and receive the chemical or physical information within the biological scope and exchange it with the electrical or information scope of the nanonetworks [226]. MC inspired by the communication among natural living entities, has the advantages of efficiency and safety in nature. Although it has the ability to transfer information over long ranges $(\mathrm{nm}-\mathrm{m})$, the speed of MC is considerably slow when the range between transceivers is longer than a few $\mu \mathrm{m}$ [224]. However, from an engineering perspective, 
how to control, modify, or reengineer the transmission of information in the natural solutions is one of the major challenges. Fortunately, engineers have recently started to analyze several different possibilities to realize MC systems. Author of [227] derived the systems-theoretic models for a diffusion-based molecular communication system design based on biological circuits. Authors of [228] proposed a new artificial molecular network architecture and employed flagellated bacteria and catalytic nanomotors as the carrier to transport the encoded DNA sequences between transmitter and receivers. Authors of [226] depicted the single-input single-output (SISO) intrabody communication between two neurons, and the multi-input single-output (MISO) synaptic communication channel. Authors of [229] investigated the transmitting rate per spike at the neuron and how to enhance the rate compared to SISO channel. A multipleaccess channel model was shown in [224], which mainly consisted of four stages to determine the communication performance.

5.3.2. Intrabody Communication within $\mathrm{THz}$ Band. The terahertz $(\mathrm{THz})$ band can be the potential communication solution in the future electromagnetic (EM) nanosensors. A $3 \mathrm{D}$ numerical model is set up in [230] to investigate the EM channel characteristics, including the path loss and noise level at $\mathrm{THz}$ frequencies which propagated within the different human tissues. The results showed that the $\mathrm{THz}$ channel attenuation versus distance was dramatically lower than the molecular situation. Then the channel capacity was studied to emphasize the possibility of applying intrabody $\mathrm{THz}$ communication systems for nanonetworks. At a distance of millimeters, the capacity can reach 100 Terabits per second (Tbps) depending on the different transmitted signal. In addition, due to the very limited capability of nanosensors, they must have simple communication and simple medium sharing mechanism. Based on On-Off Keying (OOK) protocol and Time Division Multiple Access (TDMA) framework in the THz, [225] proposed a conceptual nanonetwork model, which assumed hexagonal cell-based nanosensors deployed in cylindrical shape 3D hexagonal pole. Data transmission efficiency, for the various combinations of transmission methods, exploiting hybrid, direct, and multihop methods was analyzed in this work.

\section{Future Challenges of IBC}

From the original concept originally proposed by Zimmerman in 1996, intrabody communication has developed for more than two decades. Till now, the researchers have paid more attention to the electrical field model with analytical or numerical methods, distributed circuit model with ColeCole parameters, transceiver design and applications, in vivo and phantom experiments, and so forth. There have been important advances not only in the understanding of the bioelectric mechanism underlying IBC communication, but also in the design of more sophisticated prototypes. However, there are still some important scientific and technical challenges remaining for the successful deployment of IBC technology, such as the effect of long-term use in the human body, communication optimization through more realistic and personalized anatomical models, the study of the influence of both anthropometric characteristics and the subject's movement on the transmission performance, standardization of communications, and implementation of miniaturized and energy-efficient transceivers. IBC research has been focused on the transmission of low-rate biomedical data (ECG, blood pressure, or pulse oximetry). However, the future IBC implanted sensors will require higher data rates. For example, it has been estimated that an endoscopic capsule will generate data at a rate of $2 \mathrm{Mbps}$ while neural recording will need a sampling frequency in the range of $\mathrm{MHz}$ [231]. This remains a technical challenge for IBC researchers and designers. In addition, new IBC applications may further develop along with modern medical and health technologies, such as nanomaterials, biochemical reaction, and flexible electronics.

Implantable medicine is still an immature area, which has the potential to synthesize all these technologies together. The implanted nanosensors can gather various specific signals less invasively from organ, tissue, neuron, and even cell level. Once IBC transceivers with the nanoscale are designed to set up the nanonetworks, these nanosensors can exchange information with each other to intelligently control the release of molecular structures or certain biochemical compounds or even to stimulate certain neurons in the body. Hypothesizing, such information will be then transmitted to the wearable device outside the body which can be connected to a public network for healthcare professionals to assess, diagnose, and prevent diseases or issues at very early stage. Firstly, the fundamental work is to build the physical model in the nanoscale. The model may not be limited to the electrical scope but even combine physiological and biochemical principles in order to comprehensively clarify the mechanism for the information communication. Secondary, the nanosensors with bioelectronics interface, which can translate information from the biochemical domain to the electronics domain for simple signal processing, encoding, and translating using the IBC method, should be synthesized and realized by the nanotechnology, flexible electronics, and integrated circuit with mixed signal. In addition, how to harvest energy for the nanosensors is another key issue. Another alternative approach is to make use of the IBC weak electrical signal from the wearable device attached on the skin to charge the sensors between the biological environment and the external world. These novel approaches may open the door to an immense range of applications in medicine. Finally, the advances in the field of nanoimplantable devices will require an extensive effort in the definition of new MAC layer protocols enabling communication between implantable sensors and nanonetworks.

\section{Acronyms}

AC: Alternating current

ADC: Analog-to-digital converter

ADF: Active-digital filter

ASIC: Application-specific custom designed integrated circuits 
BAN: Body area network

BCC: Body channel communication

BER: Bit error rate

BMI: $\quad$ Body mass index

BPSK: Binary phase-shift keying

CMOS: Complementary metal-oxide-semiconductor

CPFSK: Continuous phase frequency shift keying

DCC: Duty cycle control

DCI: Direct-coupled interface

DSSS: Direct sequence spread spectrum

CIS: $\quad$ Contact impedance sensing

ECG: Electrocardiogram

EEG: Electroencephalogram

EFC: Electrostatic field communication

EM: Electromagnetic

EMG: Electromyogram

EMI: Electromagnetic interference

EMT: Electromagnetic tracking

EO: Electrical-to-optical

ET: $\quad$ Entertainment

FDTD: Finite-difference time-domain method

FEM: $\quad$ Finite element method

FM: $\quad$ Frequency modulation

FSK: $\quad$ Frequency shift keying

GC: Galvanically coupled

HAN: Human Area Networking

HBC: Human body communication

HC: Healthcare

IBC: Intrabody communication

IBCOM: Intrabrain communication

ICNIRP: International commission on nonionizing radiation protection

IL-DCO: Injection-locking digitally controlled oscillator

IR: Impulse radio

JSMEBE: Japan society of medical electronics and biological engineering

KAIST: Korea advanced institute of science and technology

MAC: Medium access control

MC: $\quad$ Molecular communications

MICS: Medical implant communication service

MISO: Multi-input single-output

MSK: Minimum shift keying

MoM: Method of moments

NRM: Noise-reduction mechanism

NRZ: Non-return-to-zero

NTT: Nippon telegraph and telephone corporation

OOK: On-Off Keying

PAN: Personal area network

PER: $\quad$ Packet error rate

PHY: $\quad$ Physical layer

PPM: $\quad$ Pulse position modulation

RF: $\quad$ Radio frequency
RSSI: Received signal strength indicator

RX: $\quad$ Receiver

SAR: $\quad$ Specific absorption rate

SISO: $\quad$ Single-input single-output

SnM: $\quad$ Synaptic nanomachine

SNR: $\quad$ Signal-to-noise ratio

Tbps: Terabits per second

TCS: Transcutaneous communication system

TDMA: Time Division Multiple Access

TX: Transmitter

VNA: Vector network analyzer

WBAN: Wireless body area network

WBS: Wideband signaling

WIBCOM: Wireless intrabrain communication

WLAN: Wireless local area network

ZDC: $\quad$ Zero delay cipher.

\section{Conflicts of Interest}

The authors declare that there are no conflicts of interest with any company or organization regarding the material discussed in this paper.

\section{Acknowledgments}

This work was supported in part by the "Fundación Progreso y Salud, Government of Andalucía," under Grants PI-00102013 and PI-0041-2014; in part by the "Fondo de Investigaciones Sanitarias, Instituto de Salud Carlos III," under Grants PI15/00306 and DTS15/00195; in part by CIBER-BBN; in part by the bilateral Croatian-Chinese project "Intrabody Communication as a Key Technology for Internet of Things in Health Applications"; in part by the National Natural Science Foundation of China U1505251, under Project of Chinese Ministry of Science and Technology 2016YFE0122700; and in part by Islamic Azad University (Urmia, Iran).

\section{References}

[1] M. Estudillo, D. Naranjo, L. M. Roa, and J. Reina-Tosina, "Intrabody communications (IBC) As an alternative proposal for biomedical wearable systems," in Handbook of Research on Developments in E-Health and Telemedicine: Technological and Social Perspectives, pp. 1-26, IGI Global, 2010.

[2] J. E. Ferguson and A. D. Redish, "Wireless communication with implanted medical devices using the conductive properties of the body," Expert Review of Medical Devices, vol. 8, no. 4, pp. 427-433, 2011.

[3] S. Warren and B. Natarajan, "Wireless communication technologies for wearable systems," Wearable Monitoring Systems, pp. 51-80, 2011.

[4] Y.-S. Lin, C.-C. Wang, Y.-C. Liao, and S.-S. Lu, "Design and implementation of intrabody communication hub/alarm unit in IBC platform for fall prevention system," Microwave and Optical Technology Letters, vol. 56, no. 10, pp. 2345-2351, 2014.

[5] T. G. Zimmerman, "Personal area networks: Near-field intrabody communication," IBM Systems Journal, vol. 35, no. 3-4, pp. 609-617, 1996. 
[6] T. I, "IEEE Standard for Local and metropolitan area networks - Part 15.6: Wireless Body Area Networks," IEEE Std, 2012.

[7] T. G. Zimmerman, Personal area networks (PAN): Near-field intra-body communication [M. S. thesis], Massachusetts Institute of Technology, 1995.

[8] C. Gabriel, S. Gabriel, and E. Corthout, "The dielectric properties of biological tissues: I. Literature survey," Physics in Medicine and Biology, vol. 41, no. 11, pp. 2231-2249, 1996.

[9] M. A. Callejón, J. Reina-Tosina, D. Naranjo-Hernandez, and L. M. Roa, "Galvanic coupling transmission in intrabody communication: A finite element approach," IEEE Transactions on Biomedical Engineering, vol. 61, no. 3, pp. 775-783, 2014.

[10] Ž. Lučev, I. Krois, and M. Cifrek, "A capacitive intrabody communication channel from $100 \mathrm{kHz}$ to $100 \mathrm{MHz}$," in Proceedings of the 2011 IEEE International Instrumentation and Measurement Technology Conference, I2MTC 2011, pp. 845-848, China, May 2011.

[11] T. Grosse-Puppendahl, S. Herber, R. Wimmer et al., "Capacitive near-field communication for ubiquitous interaction and perception," in Proceedings of the 2014 ACM International Joint Conference on Pervasive and Ubiquitous Computing, UbiComp 2014, pp. 231-242, USA, September 2014.

[12] J. J. Wikner, J. Zötterman, A. Jalili, and S. Farnebo, "Aiming for the cloud - A study of implanted battery-free temperature sensors using NFC," in Proceedings of the 2016 International Symposium on Integrated Circuits, ISIC 2016, Singapore, December 2016.

[13] M. Shinagawa, J. Katsuyama, K. Matsumoto et al., "Noise analysis for near-field intra-body communication systems," in Proceedings of the 2013 IEEE International Instrumentation and Measurement Technology Conference: Instrumentation and Measurement for Life, I2MTC 2013, pp. 902-907, USA, May 2013.

[14] M. Shinagawa, J. Katsuyama, K. Matsumoto, S. Hasegawa, R. Sugiyama, and Y. Kado, "Noise analysis for intra-body communication based on parasitic capacitance measurement," Measurement, vol. 51, no. 1, pp. 206-213, 2014.

[15] M. Shinagawa, M. Fukumoto, K. Ochiai, and H. Kyuragi, "A near-field-sensing transceiver for intrabody communication based on the electrooptic effect," IEEE Transactions on Instrumentation and Measurement, vol. 53, no. 6, pp. 1533-1538, 2004.

[16] G. S. Anderson and C. G. Sodini, "Body coupled communication: the channel and implantable sensors," in Proceedings of the IEEE International Conference on Body Sensor Networks (BSN '13), pp. 1-5, IEEE, Cambridge, Mass, USA, May 2013.

[17] J. Bae, H. Cho, K. Song, H. Lee, and H.-J. Yoo, "The signal transmission mechanism on the surface of human body for body channel communication," IEEE Transactions on Microwave Theory and Techniques, vol. 60, no. 3, pp. 582-593, 2012.

[18] M. D. Pereira, G. A. Alvarez-Botero, and F. R. De Sousa, "Characterization and Modeling of the Capacitive HBC Channel," IEEE Transactions on Instrumentation and Measurement, vol. 64, no. 10, pp. 2626-2635, 2015.

[19] R. Xu, H. Zhu, and J. Yuan, "Electric-field intrabody communication channel modeling with finite-element method," IEEE Transactions on Biomedical Engineering, vol. 58, no. 3, pp. 705712, 2011.

[20] R. Xu, W. C. Ng, H. Zhu, H. Shan, and J. Yuan, "Equation environment coupling and interference on the electric-field intrabody communication channel," IEEE Transactions on Biomedical Engineering, vol. 59, no. 7, pp. 2051-2059, 2012.

[21] E. Deventer, D. Šimunić, and M. Repacholi, EMF Standards for Human Health, Handbook of biological efects of electromagnetic fields: Biological and medical aspects of electromagnetic fields, CRC Press, Handbook of biological efects of electromagnetic fields: Biological and medical aspects of electromagnetic fields, 2007.

[22] A. Ahlbom, U. Bergqvist, J. H. Bernhardt et al., "Guidelines for limiting exposure to time-varying electric, magnetic, and electromagnetic fields (up to $300 \mathrm{GHz}$ ), Health Physics, vol. 74, no. 4, pp. 494-521, 1998.

[23] International commission on non-ionizing radiation protection, "Guidelines for limiting exposure to time-varying electric and magnetic fields (1 Hz to $100 \mathrm{kHz})$," Health Physics, vol. 100, no. 1, pp. 818-836, 2010.

[24] M. S. Wegmueller, M. Oberle, N. Felber, N. Kuster, and W. Fichtner, "Signal transmission by galvanic coupling through the human body," IEEE Transactions on Instrumentation and Measurement, vol. 59, no. 4, pp. 963-969, 2010.

[25] L. M. Roa, J. Reina-Tosina, A. Callejón-Leblic, D. Naranjo, and M. Á. Estudillo-Valderrama, "Intrabody Communication," Handbook of Biomedical Telemetry, pp. 252-300, 2014.

[26] J. Bae, K. Song, H. Lee, H. Cho, and H.-J. Yoo, "A 0.24-nJ/b wireless body-area-network transceiver with scalable doubleFSK modulation," IEEE Journal of Solid-State Circuits, vol. 47, no. 1, pp. 310-322, 2012.

[27] M. Seyedi, D. T. H. Lai, and M. Faulkner, "Limb joint effects on signal transmission in capacitive coupled intra-body communication systems," in Proceedings of the 34th Annual International Conference of the IEEE Engineering in Medicine and Biology Society, EMBS 2012, pp. 6699-6702, USA, September 2012.

[28] Ž. Lučev, I. Krois, and M. Cifrek, "Effect of body positions and movements in a capacitive intrabody communication channel from $100 \mathrm{kHz}$ to $100 \mathrm{MHz}$," in Proceedings of the 2012 IEEE International Instrumentation and Measurement Technology Conference, I2MTC 2012, pp. 2791-2795, Austria, May 2012.

[29] M. A. Callejón, D. Naranjo-Hernandez, J. Reina-Tosina, and L. M. Roa, "A comprehensive study into intrabody communication measurements," IEEE Transactions on Instrumentation and Measurement, vol. 62, no. 9, pp. 2446-2455, 2013.

[30] M. S. Wegmueller, M. Oberle, N. Kuster, and W. Fichtner, "From dielectrical properties of human tissue to intra-body communications," in Proceedings of the 10th World Congress on Medical Physics and Biomedical Engineering, WC 2006, pp. 613617, kor, September 2006.

[31] S. Gabriel, R. W. Lau, and C. Gabriel, "The dielectric properties of biological tissues: II. Measurements in the frequency range $10 \mathrm{~Hz}$ to $20 \mathrm{GHz}, "$ Physics in Medicine and Biology, vol. 41, no. 11, pp. 2251-2269, 1996.

[32] K. Partridge, B. Dahlquist, A. Veiseh et al., "Empirical measurements of intrabody communication performance under varied physical configurations," in Proceedings of the 14th Annual ACM Symposium on User Interface Software and Technology (UIST '01), pp. 183-190, ACM, November 2001.

[33] K. Hachisuka, Y. Terauchi, Y. Kishi et al., "Simplified circuit modeling and fabrication of intrabody communication devices," Sensors and Actuators A: Physical, vol. 130-131, pp. 322330, 2006.

[34] M. S. Wegmueller, A. Kuhn, J. Froehlich et al., "An attempt to model the human body as a communication channel," IEEE Transactions on Biomedical Engineering, vol. 54, no. 10, pp. 18511857, 2007.

[35] N. Cho, J. Yoo, S.-J. Song, J. Lee, S. Jeon, and H.-J. Yoo, "The human body characteristics as a signal transmission 
medium for intrabody communication," IEEE Transactions on Microwave Theory and Techniques, vol. 55, no. 5, pp. 1080-1085, 2007.

[36] Y. Song, Q. Hao, K. Zhang, M. Wang, Y. Chu, and B. Kang, "The simulation method of the galvanic coupling intrabody communication with different signal transmission paths," IEEE Transactions on Instrumentation and Measurement, vol. 60, no. 4, pp. 1257-1266, 2011.

[37] M. A. Callejón, L. M. Roa, J. Reina-Tosina, and D. NaranjoHernandez, "Study of Attenuation and Dispersion Through the Skin in Intrabody Communications Systems," IEEE Transactions on Information Technology in Biomedicine, vol. 16, no. 1, pp. 159-165, 2012.

[38] B. Kibret, M. Seyedi, D. T. H. Lai, and M. Faulkner, "Investigation of galvanic-coupled intrabody communication using the human body circuit model," IEEE Journal of Biomedical and Health Informatics, vol. 18, no. 4, pp. 1196-1206, 2014.

[39] M. Swaminathan, G. Schirner, and K. R. Chowdhury, "Optimization of energy efficient relay position for galvanic coupled intra-body communication," in Proceedings of the 2015 IEEE Wireless Communications and Networking Conference, WCNC 2015, pp. 1725-1730, USA, March 2015.

[40] H. Wang, X. Tang, C. S. Choy, and G. E. Sobelman, "Cascaded Network Body Channel Model for Intrabody Communication," IEEE Journal of Biomedical and Health Informatics, vol. 20, no. 4, pp. 1044-1052, 2016.

[41] K. Fujii, K. Ito, and S. Tajima, "A study on the receiving signal level in relation with the location of electrodes for wearable devices using human body as a transmission channel," in Proceedings of the 2003 IEEE International Antennas and Propagation Symposium and USNC/CNC/URSI North American Radio Science Meeting, pp. 1071-1074, usa, June 2003.

[42] K. Fujii, M. Takahashi, and K. Ito, "Electric field distributions of wearable devices using the human body as a transmission channel," IEEE Transactions on Antennas and Propagation, vol. 55, no. 7, pp. 2080-2087, 2007.

[43] J. H. Hwang, H. J. Myoung, T. W. Kang et al., "Reverse effect of ground electrode on the signal loss of human body communication," in Proceedings of the 2008 IEEE International Symposium on Antennas and Propagation and USNC/URSI National Radio Science Meeting, APSURSI, USA, July 2008.

[44] Ž. Lučev, I. Krois, and M. Cifrek, "Application of wireless intrabody communication system to muscle fatigue monitoring," in Proceedings of the 2010 IEEE International Instrumentation and Measurement Technology Conference, I2MTC 2010, pp. 16241627, USA, May 2010.

[45] Y. Gao, R. Ma, S. Pun, P. Mak, and M. Vai, "Measurement system with experiments for galvanic coupling type intra-body communication," in Proceedings of the 2012 5th International Conference on Biomedical Engineering and Informatics, BMEI 2012, pp. 761-764, China, October 2012.

[46] Y. Song, K. Zhang, Q. Hao, L. Hu, J. Wang, and F. Shang, "A finite-element simulation of galvanic coupling intra-body communication based on the whole human body," Sensors, vol. 12, no. 10, pp. 13567-13582, 2012.

[47] N. Haga, K. Saito, M. Takahashi, and K. Ito, "Equivalent circuit of intrabody communication channels inducing conduction currents inside the human body," IEEE Transactions on Antennas and Propagation, vol. 61, no. 5, pp. 2807-2816, 2013.

[48] J. Bae and H.-J. Yoo, "The effects of electrode configuration on body channel communication based on analysis of vertical and horizontal electric dipoles," IEEE Transactions on Microwave Theory and Techniques, vol. 63, no. 4, pp. 1409-1420, 2015.

[49] A. K. Teshome, B. Kibret, and D. T. H. Lai, "Galvanically Coupled Intrabody Communications for Medical Implants: A Unified Analytic Model," IEEE Transactions on Antennas and Propagation, vol. 64, no. 7, pp. 2989-3002, 2016.

[50] J. Park, H. Garudadri, and P. P. Mercier, "Channel Modeling of Miniaturized Battery-Powered Capacitive Human Body Communication Systems," IEEE Transactions on Biomedical Engineering, vol. 64, no. 2, pp. 452-462, 2017.

[51] M. S. Wegmueller, S. Huclova, J. Froehlich et al., "Galvanic coupling enabling wireless implant communications," IEEE Transactions on Instrumentation and Measurement, vol. 58, no. 8, pp. 2618-2625, 2009.

[52] M. A. Callejón, J. Reina-Tosina, D. Naranjo-Hernandez, and L. M. Roa, "Measurement issues in galvanic intrabody communication: Influence of experimental setup," IEEE Transactions on Biomedical Engineering, vol. 62, no. 11, pp. 2724-2732, 2015.

[53] E. R. Post, M. Reynolds, M. Gray, J. Paradiso, and N. Gershenfeld, "Intrabody buses for data and power", in Proceedings of the 1997 1st International Symposium on Wearable Computers, pp. 52-55, October 1997.

[54] M. Fukumoto and M. Shinagawa, "CarpetLAN: A Novel Indoor Wireless(-like) Networking and Positioning System," in UbiComp 2005: Ubiquitous Computing, vol. 3660 of Lecture Notes in Computer Science, pp. 1-18, Springer Berlin Heidelberg, Berlin, Heidelberg, 2005.

[55] Y. Kado and M. Shinagawa, "RedTacton near-body electricfield communications technology and its applications," NTT Technical Review, vol. 8, no. 3, 2010.

[56] Y. Kado, T. Kobase, T. Yanagawa et al., "Human-area networking technology based on near-field coupling transceiver," in Proceedings of the 2012 6th IEEE Radio and Wireless Week, RWW 2012 - 2012 IEEE Radio and Wireless Symposium, RWS 2012, pp. 119-122, USA, January 2012.

[57] Y. Kado, "Human-area networking technology as a universal interface - communications through natural human actions: Touching, holding, stepping," in Proceedings of the 2009 Symposium on VLSI Circuits, pp. 102-105, jpn, June 2009.

[58] Y. Kado, T. Yanagawa, K. Nagata et al., "Transmission characteristics between wearable and embedded transceivers based on near-field coupling: Body-channel communication system for human-area networking," in Proceedings of the 6th European Conference on Antennas and Propagation, EuCAP 2012, pp. 3130-3133, Czech Republic, March 2012.

[59] R. Nagai, T. Kobase, T. Kusunoki, H. Shimasaki, Y. Kado, and M. Shinagawa, "Near-field coupling communication technology for human-area networking, Journal of Systemics," Cybernetics and Informatics, vol. 10, no. 6, pp. 14-18, 2012.

[60] M. Ishida, T. Nakamura, M. Nozawa, N. Watanabe, Y. Kado, and M. Shinagawa, "MHz-band RF signal propagation characteristics on human body for intra-body communication," in Proceedings of the 2014 IEEE International Instrumentation and Measurement Technology Conference: Instrumentation and Measurement for Sustainable Development, I2MTC 2014, pp. 797-801, Uruguay, May 2014.

[61] K. Fujii, K. Ito, and S. Tajima, "Signal propagation of wearable computer using human body as transmission channel," in Proceedings of the in Proceedings of the International Symposium on Antennas and Propagation ISAP-02, pp. 512-515, 2002.

[62] K. Hachisuka, T. Takeda, Y. Terauchi, K. Sasaki, H. Hosaka, and K. Itao, "Intra-body data transmission for the personal area 
network," Microsystem Technologies, vol. 11, no. 8-10, pp. 10201027, 2005.

[63] K. Hachisuka, A. Nakata, T. Takeda et al., "Development and performance analysis of an intra-body communication device," in Proceedings of the 12th International Conference on Solid-State Sensors, Actuators and Microsystems, TRANSDUCERS 2003 Digest of Technical Papers, pp. 1722-1725, USA, June 2003.

[64] K. Hachisuka, A. Nakata, T. Takeda et al., "Development of wearable intra-body communication devices," Sensors and Actuators A: Physical, vol. 105, no. 1, pp. 109-115, 2003.

[65] T. C. W. Schenk, N. S. Mazloum, L. Tan, and P. Rutten, "Experimental characterization of the body-coupled communications channel," in Proceedings of the IEEE International Symposium on Wireless Communication Systems (ISWCS '08), pp. 234-239, October 2008.

[66] B. Gyselinckx, C. van Hoof, J. Ryckaert, R. F. Yazicioglu, P. Fiorini, and V. Leonov, "Human++: autonomous wireless sensors for body area networks," in Proceedings of the Custom Integrated Circuits Conference, pp. 13-19, IEEE, September 2005.

[67] J. Penders, B. Gyselinckx, R. Vullers et al., "Human++: From technology to emerging health monitoring concepts," in Proceedings of the 5th International Workshop on Wearable and Implantable Body Sensor Networks, BSN 2008, in conjunction with the 5th International Summer School and Symposium on Medical Devices and Biosensors, ISSS-MDBS 2008, pp. 94-98, China, June 2008.

[68] Ž. Lučev, I. Krois, and M. Cifrek, "A multichannel wireless EMG measurement system based on intrabody communication," in Proceedings of the 19th IMEKO World Congress 2009, pp. 17761780, prt, September 2009.

[69] Ž. Lučev, I. Krois, M. Cifrek, H. Džapo, and S. Tonković, "Effects of transmitter position and receiver ground plane on signal transmission in intrabody wireless EMG measurement system," in Proceedings of the World Congress on Medical Physics and Biomedical Engineering: Diagnostic and Therapeutic Instrumentation, Clinical Engineering, pp. 887-890, deu, September 2009.

[70] F. Grilec, Ž. Lučev Vasić, W. Ni, Y. Gao, M. Du, and M. Cifrek, "Wireless intrabody communication sensor node realized using PSoC microcontroller," in Proceedings of the 39th International Convention on Information and Communication Technology, Electronics and Microelectronics, MIPRO 2016, pp. 426-429, Croatia, June 2016.

[71] F. Grilec, A. Stanešić, Ž. Lučev Vasić, Y. Gao, M. Du, and M. Cifrek, "Single-chip intrabody communication node," in Proceedings of the International Conference on Medical and Biological Engineering, CMBEBIH 2017, pp. 305-310, Bosnia and Herzegovina, March 2017.

[72] Y. Song, K. Zhang, Q. Hao, and J. P. Rolland, "Modeling and characterization of the electrostatic coupling intra-body communication based on Mach-Zehnder electro-optical modulation," Optics Express, vol. 20, no. 12, pp. 13488-13500, 2012.

[73] Y. Song, Q. Hao, K. Zhang, J. Wang, X. Jin, and H. Sun, "Signal transmission in a human body medium-based Body Sensor Network using a Mach-Zehnder electro-optical sensor," Sensors, vol. 12, no. 12, pp. 16557-16570, 2012.

[74] Y. Song, Q. Hao, and K. Zhang, "Review of the Modeling, Simulation and Implement of Intra-body Communication," Defence Technology, vol. 9, no. 1, pp. 10-17, 2013.

[75] K. Zhang, Q. Hao, Y. Song, J. Wang, R. Huang, and Y. Liu, "Modeling and characterization of the implant intra-body communication based on capacitive coupling using a transfer function method," Sensors, vol. 14, no. 1, pp. 1740-1756, 2014.
[76] M. Li, Y. Song, G. Wang, Q. Hao, and K. Zang, "Characterization of the implantable intra-body communication based on capacitive coupling by transfer function," in Proceedings of the 10th International Conference on Sensing Technology, ICST 2016, China, November 2016.

[77] E. Okamoto, S. Kikuchi, and Y. Mitamura, "Evaluation of titanium mesh electrode using for transcutaneous intrabody communication by tissue-electrode impedance," in Proceedings of the 2013 35th Annual International Conference of the IEEE Engineering in Medicine and Biology Society, EMBC 2013, pp. 667-670, Japan, July 2013.

[78] E. Okamoto, Y. Kato, K. Seino et al., "A New Transcutaneous Bidirectional Communication for Monitoring Implanted Artificial Heart Using the Human Body as a Conductive Medium," Artificial Organs, vol. 36, no. 10, pp. 852-858, 2012.

[79] Z. D. Nie, J. J. Ma, Z. C. Li, H. Chen, and L. Wang, "Dynamic propagation channel characterization and modeling for human body communication," Sensors, vol. 12, no. 12, pp. 17569-17587, 2012.

[80] Z. Nie, J. Ma, I. Kamen, and L. Wang, "An investigation on dynamic human body communication channel characteristics at $45 \mathrm{MHz}$ in different surrounding environments," IEEE Antennas and Wireless Propagation Letters, vol. 13, pp. 309-312, 2014.

[81] T. Leng, Z. Nie, W. Wang, F. Guan, and L. Wang, "A human body communication transceiver based on on-off keying modulation," in Proceedings of the 2nd International Symposium on Bioelectronics and Bioinformatics (ISBB '11), pp. 61-64, November 2011.

[82] J. Li, Z. Nie, Y. Liu, L. Wang, and Y. Hao, "Characterization of In-Body Radio Channels for Wireless Implants," IEEE Sensors Journal, vol. 17, no. 5, pp. 1528-1537, 2017.

[83] Y. Gao, L. Zhang, M. Du, and M. I. Vai, "Design of human motion detection based on the human body communication," in Proceedings of the 35th IEEE Region 10 Conference, TENCON 2015, Macao, November 2015.

[84] W. Ni, Y. Gao, Ž. Lučev Vasić et al., "Human posture detection based on human body communication with muti-carriers modulation," in Proceedings of the 39th International Convention on Information and Communication Technology, Electronics and Microelectronics, MIPRO 2016, pp. 273-276, Croatia, June 2016.

[85] J. Shi and J. Wang, "Dual-mode impulse radio ultra-wideband transmission for body area networks," IET Microwaves, Antennas \& Propagation, vol. 5, no. 10, pp. 1250-1255, 2011.

[86] K. Shikada and J. Wang, "Development of human body communication transceiver based on impulse radio scheme," in Proceedings of the 2012 2nd IEEE CPMT Symposium Japan, ICSJ 2012, Japan, December 2012.

[87] J. Wang, T. Fujiwara, T. Kato, and D. Anzai, "Wearable ECG Based on Impulse-Radio-Type Human Body Communication," IEEE Transactions on Biomedical Engineering, vol. 63, no. 9, pp. 1887-1894, 2016.

[88] J. Mao, B. Zhao, Y. Lian, and H. Yang, "A self-adaptive body channel communication scheme for backward path loss reduction," in Proceedings of the 2016 IEEE International Symposium on Circuits and Systems, ISCAS 2016, pp. 2034-2037, Canada, May 2016.

[89] J. Mao, H. Yang, Y. Lian, and B. Zhao, "A self-adaptive capacitive compensation technique for body channel communication," IEEE Transactions on Biomedical Circuits and Systems, vol. 11, no. 5, pp. 1001-1012, 2017. 
[90] T. Handa, S. Shoji, S. Ike, S. Takeda, and T. Sekiguchi, "Very lowpower consumption wireless ECG monitoring system using body as a signal transmission medium," in Proceedings of the 1997 International Conference on Solid-State Sensors and Actuators. Part 2 (of 2), pp. 1003-1006, June 1997.

[91] D. P. Lindsey, E. L. McKee, M. L. Hull, and S. M. Howell, "A new technique for transmission of signals from implantable transducers," IEEE Transactions on Biomedical Engineering, vol. 45, no. 5, pp. 614-619, 1998.

[92] M. Oberle, Low power system-on-chip for biomedical application [Ph.D. thesis], ETH Zurich, Zurich, Switzerland, 2002, Ph.D. dissertation.

[93] M. S. Wegmüller, Intra-body communication for biomedical sensor networks [Ph.D. thesis], Zurich, Zurich, Switzerland, 2007.

[94] M. S. Wegmüller, M. Oberle, N. Kuster, and W. Fichtner, "From dielectrical properties of human tissue to intra-body communications," in in World Congress on Medical Physics and Biomedical Engineering, vol. no. 7, pp. 613-617, 2006.

[95] Z. Cai, M. Seyedi, W. Zhang, F. Rivet, and D. T. H. Lai, "Characterization of Impulse Radio Intrabody Communication System for Wireless Body Area Networks," Journal of Medical and Biological Engineering, vol. 37, no. 1, pp. 74-84, 2017.

[96] M. Seyedi, Z. Cai, D. T. H. Lai, and F. Rivet, "An energy-efficient pulse position modulation transmitter for galvanic intrabody communications," in Proceedings of the 4th International Conference on Wireless Mobile Communication and Healthcare, MOBIHEALTH 2014, pp. 192-195, grc, November 2014.

[97] S.-J. Song, N. Cho, and H.-J. Yoo, "A 0.2-mW 2-Mb/s digital transceiver based on wideband signaling for human body communications," IEEE Journal of Solid-State Circuits, vol. 42, no. 9, pp. 2021-2032, 2007.

[98] N. Cho, L. Yan, J. Bae, and H.-J. Yoo, "A 60 kb/s-10 Mb/s adaptive frequency hopping transceiver for interference-resilient body channel communication," IEEE Journal of Solid-State Circuits, vol. 44, no. 3, pp. 708-717, 2009.

[99] N. Cho, J. Bae, and H.-J. Yoo, "A 10.8 mW body channel communication/MICS dual-band transceiver for a unified body sensor network controller," IEEE Journal of Solid-State Circuits, vol. 44, no. 12, pp. 3459-3468, 2009.

[100] J. Bae, K. Song, H. Lee, H. Cho, and H.-J. Yoo, "A low-energy crystal-less double-FSK sensor node transceiver for wireless body-area network," IEEE Journal of Solid-State Circuits, vol. 47, no. 11, pp. 2678-2692, 2012.

[101] H. Lee, K. Lee, S. Hong et al., "A 5.5mW IEEE-802.15.6 wireless body-area-network standard transceiver for multichannel electro-acupuncture application," in Proceedings of the 2013 60th IEEE International Solid-State Circuits Conference, ISSCC 2013, pp. 452-453, USA, February 2013.

[102] H. Lee, H. Cho, and H.-J. Yoo, "A $33 \mu \mathrm{W} /$ node Duty Cycle Controlled HBC Transceiver system for medical BAN with 64 sensor nodes," in Proceedings of the 36th Annual Custom Integrated Circuits Conference - The Showcase for Integrated Circuit Design in the Heart of Silicon Valley, CICC 2014, USA, September 2014.

[103] H. Cho, H. Lee, J. Bae, and H.-J. Yoo, "A 5.2 mW IEEE 802.15.6 HBC Standard Compatible Transceiver with Power Efficient Delay-Locked-Loop Based BPSK Demodulator," IEEE Journal of Solid-State Circuits, vol. 50, no. 11, pp. 2549-2559, 2015.

[104] H. Cho, H. Kim, M. Kim, J. Jang, J. Bae, and H.-J. Yoo, “A 79 pJ/b $80 \mathrm{Mb} / \mathrm{s}$ full-duplex transceiver and a $42.5 \mu \mathrm{W} 100 \mathrm{~kb} / \mathrm{s}$ superregenerative transceiver for body channel communication," in
Proceedings of the 2015 62nd IEEE International Solid-State Circuits Conference, ISSCC 2015 - Digest of Technical Papers, pp. 380-381, USA, February 2015.

[105] H. Cho, H. Kim, M. Kim, J. Jang, and H. Yoo, "79 pJ/b 80 Mb/s full-duplex transceiver and $42.5 \mu \mathrm{W} 100 \mathrm{~kb} / \mathrm{s}$ super-regenerative transceiver for body channel communication," in Proceedings of the 2015 IEEE MTT-S 2015 International Microwave Workshop Series on RF and Wireless Technologies for Biomedical and Healthcare Applications (IMWS-BIO), pp. 14-15, Taipei, Taiwan, September 2015.

[106] H. Cho, H. Kim, M. Kim et al., "A 79 pJ/b $80 \mathrm{Mb} / \mathrm{s}$ fullduplex transceiver and a $42.5 \mu \mathrm{W} 100 \mathrm{~kb} / \mathrm{s}$ super-regenerative transceiver for body channel communication," IEEE Journal of Solid-State Circuits, vol. 51, no. 1, pp. 310-317, 2016.

[107] A. Fazzi, S. Ouzounov, and J. van den Homberg, "A $2.75 \mathrm{~mW}$ wideband correlation-based transceiver for body-coupled communication," in Proceedings of the IEEE International Solid-State Circuits Conference-Digest of Technical Papers (ISSCC '09), pp. 204-205, San Francisco, Calif, USA, February 2009.

[108] Y.-T. Lin, Y.-S. Lin, C.-H. Chen, H.-C. Chen, Y.-C. Yang, and S.-S. Lu, "A 0.5-V biomedical system-on-a-chip for intrabody communication system," IEEE Transactions on Industrial Electronics, vol. 58, no. 2, pp. 690-699, 2011.

[109] P.-Y. Tsai, S.-Y. Hsu, J.-S. Chang, T.-W. Chen, and C.-Y. Lee, "A QPSK/16-QAM OFDM-based 29.1Mbps LINC transmitter for Body Channel Communication," in Proceedings of the 2012 IEEE Asian Solid-State Circuits Conference, A-SSCC 2012, pp. 345348, Japan, November 2012.

[110] P.-Y. Tsai, Y.-Y. Chang, S.-Y. Hsu, and C.-Y. Lee, "An OFDMbased 29.1Mbps $0.22 \mathrm{~nJ} /$ bit body channel communication baseband transceiver," in Proceedings of the 2015 International Symposium on VLSI Design, Automation and Test, VLSI-DAT 2015, Taiwan, April 2015.

[111] Y. Tseng, T. Lin, S. Yau, Y. Ho, and C. Su, "A 0.5V/22 fi W low power transceiver ic for use in intra-body communication system," in Proceedings of the 13th International SoC Design Conference, ISOCC 2016, pp. 71-72, Republic of Korea, October 2016.

[112] J. Lee, V. V. Kulkarni, C. K. Ho et al., "A $60 \mathrm{Mb} / \mathrm{s}$ wideband BCC transceiver with $150 \mathrm{pJ} / \mathrm{b} \mathrm{RX}$ and $31 \mathrm{pJ} / \mathrm{b}$ TX for emerging wearable applications," in Proceedings of the 2014 61st IEEE International Solid-State Circuits Conference, ISSCC 2014, pp. 498-499, USA, February 2014.

[113] H. Wang, X. Tang, C. S. Choy, K. N. Leung, and K. P. Pun, "A 5.4-mW 180-cm Transmission Distance 2.5-Mb/s Advanced Techniques-Based Novel Intrabody Communication Receiver Analog Front End," IEEE Transactions on Very Large Scale Integration (VLSI) Systems, vol. 23, no. 12, pp. 2829-2841, 2015.

[114] Y. L. Tsou, C.-S. A. Gong, N. C. Cheng, Y. Lee, and C. F. Jou, "Integrated biosensing platform based on a 1.74-mW -90- dBm sensitivity dual- mode-operation receiver for IEEE 802.15.6 human body communication standard," IEEE Sensors Journal, vol. 15, no. 6, pp. 3317-3327, 2015.

[115] W. Saadeh, Y. Kifle, and J. Y. Masdar, "A hybrid OFDM body coupled communication transceiver for binaural hearing AIDS in 65nm CMOS," in Proceedings of the IEEE International Symposium on Circuits and Systems, ISCAS 2015, pp. 2620-2623, Portugal, May 2015.

[116] W. Saadeh, M. A. B. Altaf, H. Alsuradi, and J. Yoo, "A Pseudo OFDM with Miniaturized FSK Demodulation Body-Coupled Communication Transceiver for Binaural Hearing AIDS in 65 
nm CMOS," IEEE Journal of Solid-State Circuits, vol. 52, no. 3, pp. 757-768, 2017.

[117] W. Saadeh, H. Alsuradi, M. A. B. Altaf, and J. Yoo, "A 1.1mW hybrid OFDM ground effect-resilient body coupled communication transceiver for head and body area network," in Proceedings of the 12th IEEE Asian Solid-State Circuits Conference, ASSCC 2016, pp. 201-204, Japan, November 2016.

[118] W. Saadeh, M. A. B. Altaf, H. Alsuradi, and J. Yoo, "A 1.1$\mathrm{mW}$ Ground Effect-Resilient Body-Coupled Communication Transceiver with Pseudo OFDM for Head and Body Area Network," IEEE Journal of Solid-State Circuits, vol. 52, no. 10, pp. 2690-2702, 2017.

[119] C.-C. Chung, C.-T. Chang, and C.-Y. Lin, "A $1 \mathrm{Mb} / \mathrm{s}-40 \mathrm{Mb} / \mathrm{s}$ human body channel communication transceiver," in Proceedings of the 2015 International Symposium on VLSI Design, Automation and Test, VLSI-DAT 2015, Taiwan, April 2015.

[120] C. Chung, C. Lin, and J. Yang, "Time-domain characteristics of body channel communication (BCC) and BCC transceiver design," in Proceedings of the 2016 International Symposium on VLSI Design, Automation and Test (VLSI-DAT), pp. 1-4, Hsinchu, Taiwan, April 2016.

[121] C.-C. Chung, Y.-C. Tsai, and M.-C. Li, "A reference-less alldigital transceiver for human body channel communication," in Proceedings of the 2017 International Symposium on VLSI Design, Automation and Test, VLSI-DAT 2017, Taiwan, April 2017.

[122] K. Lin, B. Wang, X. Zhang, X. Wang, T. Ouyang, and H. Chen, "A novel low-power compact WBS human body channel receiver for wearable vital signal sensing application in wireless bodyarea network," Microsystem Technologies, vol. 23, no. 10, pp. 4459-4473, 2017.

[123] K. R. Foster and H. P. Schwan, "Dielectric properties of tissues and biological materials: a critical review," Critical Reviews in Biomedical Engineering, vol. 17, no. 1, pp. 25-104, 1989.

[124] K. S. Cole, "Membranes, ions, and impulses: A chapter of classical biophysics," University of California Press, vol. 5, 1972.

[125] M. R. Basar, M. Y. Ahmad, J. Cho, and F. Ibrahim, "Application of wireless power transmission systems in wireless capsule endoscopy: An overview," Sensors, vol. 14, no. 6, pp. 1092910951, 2014.

[126] B. Liu and X. Guo, "Introduction and comparsion of domesic and foreign electromagnetic radiation protection standard," Journal of Radiological Protection, pp. 51-56, 2008.

[127] "Japan Society of Medical Electronics and Biological Engineering," in In basic knowledge and safety management of medical and biological engineering, pp. 42-60, Nankodo Co. Ltd., Tokyo, Japan, 4th edition, 2002.

[128] Ž. Lučev, A. Koričan, and M. Cifrek, "A finite element method study of the current density distribution in a capacitive intrabody communication system," in Proceedings of the 12th Mediterranean Conference on Medical and Biological Engineering and Computing, MEDICON 2010, pp. 422-425, May 2010.

[129] X. Zeng, Y. Gao, S. Pan, B. Mai, M. Wei, and M. Du, "Effects of muscle conductivity on signal transmission of intra-body communications," Journal of Electronic Measurement and Instrument, vol. 27, no. 1, pp. 21-25, 2013.

[130] M. Swaminathan, F. S. Cabrera, J. S. Pujol, U. Muncuk, G. Schirner, and K. R. Chowdhury, "Multi-Path Model and Sensitivity Analysis for Galvanic Coupled Intra-Body Communication Through Layered Tissue," IEEE Transactions on Biomedical Circuits and Systems, vol. 10, no. 2, pp. 339-351, 2016.
[131] K. Ito and Y. Hotta, "Signal Path Loss Simulation of Human Arm for Galvanic Coupling Intra-Body Communication Using Circuit and Finite Element Method Models," in Proceedings of the 2015 12th IEEE International Symposium on Autonomous Decentralized Systems, ISADS 2015, pp. 230-235, Taiwan, March 2015.

[132] M. A. Callejón, D. Naranjo-Hernández, J. Reina-Tosina, and L. M. Roa, "Distributed circuit modeling of galvanic and capacitive coupling for intrabody communication," IEEE Transactions on Biomedical Engineering, vol. 59, no. 12, pp. 3263-3269, 2012.

[133] N. Haga, K. Motojima, M. Shinagawa, and Y. Kado, "EquivalentCircuit Expression of Environmental Noise Electric Fields in Intrabody Communication Channels," IEEE Transactions on Electromagnetic Compatibility, vol. 58, no. 1, pp. 294-306, 2016.

[134] Y.-M. Gao, Z.-M. Wu, S.-H. Pun, P.-U. Mak, M.-I. Vai, and M. Du, "A novel field-circuit FEM modeling and channel gain estimation for galvanic coupling real IBC measurements," Sensors, vol. 16, no. 4, article 471, 2016.

[135] M. A. Callejón, P. del Campo, J. Reina-Tosina, and L. M. Roa, "A Parametric Computational Analysis into Galvanic Coupling Intrabody Communication," IEEE Journal of Biomedical and Health Informatics, 2017.

[136] Y.-M. Gao, S. H. Pun, M. Du, M. I. Vai, and P. U. Mak, "A Preliminary two dimensional model for Intra-body communication of body sensor networks," in Proceedings of the 2008 International Conference on Intelligent Sensors, Sensor Networks and Information Processing, ISSNIP 2008, pp. 273-278, Australia, December 2008.

[137] S. H. Pun, Y. M. Gao, P. U. Mak, M. Du, and M. I. Vai, "Modeling for intra-body communication with bone effect," in Proceedings of the 31st Annual International Conference of the IEEE Engineering in Medicine and Biology Society: Engineering the Future of Biomedicine, EMBC 2009, pp. 693-696, USA, September 2009.

[138] S. H. Pun, Y. M. Gao, P. U. Mak, M. I. Vai, and M. Du, "Quasistatic multilayer electrical modeling of human limb for IBC," in Proceedings of the 14th World Multi-Conference on Systemics, Cybernetics and Informatics, WMSCI 2010, pp. 304-309, usa, July 2010.

[139] S. H. Pun, Y. M. Gao, P. Mak, M. I. Vai, and M. Du, "Quasi-static modeling of human limb for intra-body communications with experiments," IEEE Transactions on Information Technology in Biomedicine, vol. 15, no. 6, pp. 870-876, 2011.

[140] F. Koshiji, K. Sasaki, D. Muramatsu, and K. Koshiji, "Input impedance characteristics of wearable transmitter electrodes for intra-body communication," in Proceedings of the 1st IEEE Global Conference on Consumer Electronics (GCCE '12), pp. 362363, October 2012.

[141] A. Alshehab, N. Kobayashi, J. Ruiz, R. Kikuchi, S. Shimamoto, and H. Ishibashi, "A study on intrabody communication for personal healthcare monitoring system," Telemedicine and $e$ Health, vol. 14, no. 8, pp. 851-857, 2008.

[142] B. Kibret, M. Seyedi, D. T. H. Lai, and M. Faulkner, "The effect of tissues in galvanic coupling intrabody communication," in Proceedings of the IEEE 8th International Conference on Intelligent Sensors, Sensor Networks and Information Processing: Sensing the Future (ISSNIP '13), pp. 318-323, April 2013.

[143] C. O. Asogwa, S. F. Collins, P. McLaughlin, and D. T. H. Lai, "A galvanic coupling method for assessing hydration rates," Electronics, vol. 5, no. 3, 2016.

[144] I. Yokota, H. Shimasaki, T. Nakamura, Y. Kado, and M. Ishida, "Analysis of signal propagation loss on the human body in 
intrabody communication," in Proceedings of the 9th International Symposium on Medical Information and Communication Technology, ISMICT 2015, pp. 35-39, Japan, March 2015.

[145] X. M. Chen, S. H. Pun, Z. T. Wang, P.-U. Mak, and M. I. Vai, "Quasi-static field modeling with bone and joint effects in intrabody communication," in Proceedings of the 8th International Conference on Information, Communications and Signal Processing, ICICS 2011, Singapore, December 2011.

[146] Ž. Lučev, I. Krois, and M. Cifrek, "A capacitive intrabody communication channel from $100 \mathrm{kHz}$ to $100 \mathrm{MHz}$," IEEE Transactions on Instrumentation and Measurement, vol. 61, no. 12, pp. 3280-3289, 2012.

[147] K. Zhao, Z. Ying, and S. He, "Intrabody Communications Between Mobile Device and Wearable Device at $26 \mathrm{MHz}$," IEEE Antennas and Wireless Propagation Letters, vol. 16, pp. 549-552, 2017.

[148] T. Ohishi, K. Inoue, and S. Obayashi, "Novel pair electrode with coils sensing magnetic energy on human body surface for intrabody communication," in Proceedings of the 2012 17th International Symposium on Antennas and Propagation, ISAP 2012, pp. 203-206, jpn, November 2012.

[149] J. A. Ruiz, J. Xu, and S. Shimamoto, "Propagation characteristics of intra-body communications for body area networks," in Proceedings of the 3rd IEEE Consumer Communications and Networking Conference (CCNC '06), vol. 1, pp. 509-513, January 2006.

[150] A. E. Khorshid, A. M. Eltawil, and F. Kurdahi, "Intra-body communication model based on variable biological parameters," in Proceedings of the 49th Asilomar Conference on Signals, Systems and Computers, ACSSC 2015, pp. 948-951, USA, November 2015.

[151] M. Seyedi and D. T. H. Lai, "Effect of limb joints and limb movement on intrabody communications for body area Network applications," Journal of Medical and Biological Engineering, vol. 34, no. 3, pp. 276-283, 2014.

[152] Ž. Lučev Vasić, I. Krois, and M. Cifrek, "On a pulse response of a capacitive intrabody communication channel," in Proceedings of the IEEE EuroCon 2013, pp. 1785-1789, Croatia, July 2013.

[153] A. Ruaro, J. Thaysen, and K. B. Jakobseny, "Head-centric bodychannel propagation paths characterization," in Proceedings of the 9th European Conference on Antennas and Propagation, EuCAP 2015, prt, May 2015.

[154] M. D. Pereira, K. T. Silvestri, and F. R. De Sousa, "Measurement results and analysis on a HBC channel," in Proceedings of the 9th IEEE International Symposium on Medical Measurements and Applications, IEEE MeMeA 2014, Portugal, June 2014.

[155] H. Elayan and R. M. Shubair, "On channel characterization in human body communication for medical monitoring systems," in Proceedings of the 17th International Symposium on Antenna Technology and Applied Electromagnetics, ANTEM 2016, Canada, July 2016.

[156] L. Yang, J. Kuang, Z. He, and F. Jun, "Measurement system for propagation characteristics of intra-body communication," in Proceedings of the 2010 6th International Conference on Wireless Communications, Networking and Mobile Computing, WiCOM 2010, China, September 2010.

[157] T. X. Nguyen, D. Muramatsu, and K. Sasaki, "Measurement of human body communication transmission characteristics at 20 $\mathrm{MHz}$, in Proceedings of the 9th International Symposium on Medical Information and Communication Technology, ISMICT 2015, pp. 45-48, Japan, March 2015.
[158] A. H. A. Razak, A. Zayegh, R. K. Begg, M. Seyedi, and D. T. Lai, "BFSK modulation to compare intra-body communication methods for foot plantar pressure measurement," in Proceedings of the 2013 7th IEEE GCC Conference and Exhibition, GCC 2013, pp. 172-176, Qatar, November 2013.

[159] Y. Kifle, H.-S. Kim, and J. Yoo, "Human body and head characteristics as a communication medium for Body Area Network," in Proceedings of the 37th Annual International Conference of the IEEE Engineering in Medicine and Biology Society, EMBC 2015, pp. 1845-1848, Italy, August 2015.

[160] H. Zhu, W. C. Ng, H. Shan, and J. Yuan, "A physical layer security analysis on the electric-field intra-body communication," in Proceedings of the 2012 International Conference on Computing, Networking and Communications, ICNC'12, pp. 14-17, USA, February 2012.

[161] R. Xu, H. Zhu, and J. Yuan, "Characterization and analysis of intra-body communication channel," in Proceedings of the 2009 IEEE International Symposium on Antennas and Propagation and USNC/URSI National Radio Science Meeting, APSURSI 2009, USA, June 2009.

[162] S. H. Pun, Y. M. Gao, P. U. Mak, M. I. Vai, and M. Du, “The physical layer design of intra-body communication: Model, transmitter and receiver," in Proceedings of the 2011 IEEE International Conference on Consumer Electronics, ICCE 2011, pp. 605-606, Las Vegas, Nev, USA, January 2011.

[163] L.-S. Wu, J. Sakai, H.-C. Sun, and Y.-X. Guo, "Matching network to improve the transmission level of capacitive intrabody communication (IBC) channels," in Proceedings of the 2013 IEEE MTT-S International Microwave Workshop Series on $R F$ and Wireless Tecbhnologies for Biomedical and Healthcare Applications, IMWS-BIO 2013, Singapore, December 2013.

[164] J. Rosell, J. Colominas, P. Riu, R. Pallas-Areny, and J. G. Webster, "Skin Impedance From $1 \mathrm{~Hz}$ to $1 \mathrm{MHz}$, IEEE Transactions on Biomedical Engineering, vol. 35, no. 8, pp. 649-652, 1988.

[165] D. Muramatsu, F. Koshiji, K. Koshiji, and K. Sasaki, "Input impedance analysis of wearable antenna and its experimental study with real human body," in Proceedings of the 2014 IEEE International Conference on Consumer Electronics, ICCE 2014, pp. 151-152, USA, January 2014.

[166] M. A. Callejón, D. Naranjo, L. J. Reina-Tosina, and L. M. Roa, "A first approach to the harmonization of intrabody communications measurements," in Proceedings of the World Congress on Medical Physics and Biomedical Engineering, pp. 704-707, Beijing, China, May 2012.

[167] J. A. Ruiz and S. Shimamoto, "Statistical Modeling of Intra-body Propagation Channel," in Proceedings of the 2007 IEEE Wireless Communications and Networking Conference, pp. 2063-2068, Kowloon, China, March 2007.

[168] M. Seyedi, B. Kibret, D. T. H. Lai, and M. Faulkner, "An empirical comparison of limb joint effects on capacitive and galvanic coupled intra-body communications," in Proceedings of the 2013 IEEE 8th International Conference on Intelligent Sensors, Sensor Networks and Information Processing: Sensing the Future, ISSNIP 2013, pp. 213-218, Australia, April 2013.

[169] Y. M. Gao, X. X. Li, Ž. Lučev Vasić et al., "Channel modeling and simulation for galvanic coupling intra-body communication," in Proceedings of the 15th International Conference on Biomedical Engineering, ICBME 2013, pp. 655-658, Singapore, December 2013.

[170] Y. M. Gao, Y. T. Ye, M. I. Vai, M. Du, and S. H. Pun, "Channel modeling and power consumption analysis for galvanic coupling intra-body communication," EURASIP Journal on 
Wireless Communications and Networking, vol. 2016, no. 1, article no. 106, 2016.

[171] I. Yokota, M. Ishida, H. Shimasaki, and Y. Kado, "Analysis of dependence of signal propagation loss on poses in intra-body communication," in Proceedings of the 9th European Conference on Antennas and Propagation, EuCAP 2015, prt, May 2015.

[172] M. I. Kazim, M. I. Kazim, and J. J. Wikner, "An efficient full-wave electromagnetic analysis for capacitive body-coupled communication," International Journal of Antennas and Propagation, vol. 2015, Article ID 245621, 2015.

[173] M. I. Kazim, M. I. Kazim, and J. J. Wikner, "Complex path impedance estimation and matching requirements for bodycoupled communication," in Proceedings of the European Conference on Circuit Theory and Design, ECCTD 2015, Norway, August 2015.

[174] X. M. Chen, S. H. Pun, J. F. Zhao, P. U. Mak, B. D. Liang, and M. I. Vai, "Effects of human limb gestures on galvanic coupling intra-body communication for advanced healthcare system," Biomedical Engineering Online, vol. 15, no. 1, article no. 60, 2016.

[175] K. Sasaki, N. Arai, D. Muramatsu, and F. Koshiji, "Evaluation of Ground Loop Through the Floor in Human Body Communication," International Journal of Wireless Information Networks, vol. 24, no. 2, pp. 78-90, 2017.

[176] Ž. Lučev, I. Krois, and M. Cifrek, "Intrabody Communication in Biotelemetry," in Wearable and Autonomous Biomedical Devices and Systems for Smart Environment, vol. 75 of Lecture Notes in Electrical Engineering, pp. 351-368, Springer Berlin Heidelberg, Berlin, Heidelberg, 2010.

[177] J.-P. M. G. Linnartz, "Rules of thumb for predicting path loss in body coupled communication channels," in Proceedings of the 20th IEEE Symposium on Communications and Vehicular Technology in the BeNeLux, IEEE SCVT 2013, Belgium, November 2013.

[178] C. Aliau-Bonet and R. Pallas-Areny, "On the effect of body capacitance to ground in tetrapolar bioimpedance measurements," IEEE Transactions on Biomedical Engineering, vol. 59, no. 12, pp. 3405-3411, 2012.

[179] S. Grimnes and Ø. G. Martinsen, "Sources of error in tetrapolar impedance measurements on biomaterials and other ionic conductors," Journal of Physics D: Applied Physics, vol. 40, no. 1, article no. S02, pp. 9-14, 2007.

[180] A.-I. Sasaki, T. Mizota, H. Morimura et al., "Extended noisychannel models for capacitively coupled personal area network under influence of a wall," IEEE Transactions on Antennas and Propagation, vol. 62, no. 5, pp. 2802-2822, 2014.

[181] X. M. Chen, S. H. Pun, Y. M. Gao, P. U. Mak, M. I. Vai, and M. $\mathrm{Du}$, "Study on transfer function of intra-body communication based on quasi-static electric field modeling," in Proceedings of the IEEE-EMBS International Conference on Biomedical and Health Informatics, BHI 2012. In Conj. with the 8th Int. Symp.on Medical Devices and Biosensors and the 7th Int. Symp. on Biomedical and Health Engineering, pp. 388-391, China, January 2012.

[182] J.-H. Hwang, T.-W. Kang, J.-H. Kwon, and S.-O. Park, “Effect of Electromagnetic Interference on Human Body Communication," IEEE Transactions on Electromagnetic Compatibility, vol. 59, no. 1, pp. 48-57, 2017.

[183] Y. Hayashida, M. Hasegawa, A. Suzuki, M. Shinagawa, Y. Kado, and N. Haga, "Radiated noise analysis via human body for intrabody communication," Measurement, vol. 89, pp. 159-165, 2016.

[184] N. Haga and K. Motojima, "Noise sources in equivalent circuits of intrabody communication channels," in Proceedings of the
2013 International Workshop on Antenna Technology, iWAT 2013, pp. 257-260, Germany, March 2013.

[185] Y. Hayashida, R. Sugiyama, Y. Ido et al., "Capacitance model of embedded transceiver for intra-body communication," in Proceedings of the 9th International Conference on Body Area Networks, BODYNETS 2014, pp. 222-228, UK, October 2014.

[186] M. Seyedi, B. Kibret, D. T. H. Lai, and M. Faulkner, "A survey on intrabody communications for body area network applications," IEEE Transactions on Biomedical Engineering, vol. 60, no. 8, pp. 2067-2079, 2013.

[187] Ž. Lučev Vasić, Y. M. Gao, S. H. Pun et al., "Effect of transmitter and receiver electrodes configurations on the capacitive intrabody communication channel from $100 \mathrm{KHz}$ to $100 \mathrm{MHz}$," in Proceedings of the 15th International Conference on Biomedical Engineering, ICBME 2013, pp. 613-616, Singapore, December 2013.

[188] Ž. Lučev Vasić, I. Krois, and M. Cifrek, "Effect of transformer symmetry on intrabody communication channel measurements using grounded instruments," Automatika - Journal for Control, Measurement, Electronics, Computing and Communications, vol. 57, no. 1, pp. 15-26, 2016.

[189] M. Fukumoto and Y. Tonomura, "Body coupled FingeRing: Wireless wearable keyboard," in Proceedings of the 1997 Conference on Human Factors in Computing Systems, CHI, pp. 147-154, March 1997.

[190] K. Katsu, D. Anzai, and J. Wang, "Performance evaluation on correlation detection and energy detection for ultra widebandimpulse radio communication with multi-pulse position modulation scheme in implant body area networks," IET Communications, vol. 7, no. 13, pp. 1430-1436, 2013.

[191] H.-J. Yoo, "Wireless body area network and its healthcare applications," in Proceedings of the 2013 3rd Asia-Pacific Microwave Conference, APMC 2013, pp. 89-91, Republic of Korea, November 2013.

[192] S.-J. Song, S. J. Lee, N. Cho, and H.-J. Yoo, "Low power wearable audio player using human body communications," in Proceedings of the 10th IEEE International Symposium on Wearable Computers, ISWC 2006, pp. 125-126, Switzerland, October 2006.

[193] S.-J. Song, N. Cho, S. Kim, J. Yoo, S. Choi, and H.-J. Yoo, "A 0.9V $2.6 \mathrm{~mW}$ body-coupled scalable PHY transceiver for body sensor applications," in Proceedings of the 54th IEEE International Solid-State Circuits Conference, ISSCC 2007, pp. 355-609, USA, February 2007.

[194] J. Bae, K. Song, H. Lee, H. Cho, L. Yan, and H.-J. Yoo, "A 0.24nJ/b wireless body-area-network transceiver with scalable doubleFSK modulation," in Proceedings of the 2011 IEEE International Solid-State Circuits Conference, ISSCC 2011, pp. 34-35, USA, February 2011.

[195] H. Cho, H. Lee, J. Bae, and H.-J. Yoo, “A 5.2mW IEEE 802.15.6 HBC standard compatible transceiver with power efficient delay-locked-loop based BPSK demodulator," in Proceedings of the 2014 10th IEEE Asian Solid-State Circuits Conference, ASSCC 2014, pp. 297-300, Taiwan, November 2014.

[196] P. Harikumar, M. I. Kazim, and J. J. Wikner, "An analog receiver front-end for capacitive body-coupled communication," in Proceedings of the NORCHIP Conference, pp. 1-4, IEEE, Cpenhagen, Denmark, November 2012.

[197] Y. Liu, Y. Yang, D. Li, and Z. Shi, "An IM2-free floating current buffer using average power based automatic calibration for IEEE 802.15.6 transmitter," IEICE Electronics Express, vol. 13, no. $16,2016$. 
[198] P. Chen, H. Yang, R. Luo, and B. Zhao, "All-Digital GalvanicallyCoupled BCC Receiver Resilient to Frequency Misalignment," IEEE Transactions on Biomedical Circuits and Systems, vol. 11, no. 3, pp. 714-726, 2017.

[199] K. M. Al-Ashmouny, C. Boldt, J. E. Ferguson, A. G. Erdman, A. Redish, and E. Yoon, "IBCOM (intra-brain communication) microsystem: wireless transmission of neural signals within the brain.," Conference proceedings: IEEE Engineering in Medicine and Biology Society, pp. 2054-2057, 2009.

[200] K. Sasagawa, T. Matsuda, P. Davis et al., "Wireless intrabrain communication for image transmission through mouse brain.," Conference proceedings: IEEE Engineering in Medicine and Biology Society, vol. 2011, pp. 2917-2920, 2011.

[201] H. Hayami, H. Takehara, K. Nagata et al., "Wireless image-data transmission from an implanted image sensor through a living mouse brain by intra body communication," Japanese Journal of Applied Physics, vol. 55, no. 4, Article ID 04EM03, 2016.

[202] J. A. Ruiz and S. Shimamoto, "Experimental evaluation of body channel response and digital modulation schemes for intrabody communications," in Proceedings of the IEEE International Conference on Communications (ICC '06), vol. 1, pp. 349-354, IEEE, Istanbul, Turkey, June 2006.

[203] M. S. Wegmüller, M. Oberle, N. Felber, N. Kuster, and W. Fichtner, "Digital data communication through the human body for biomedical monitoring sensor," in in World Congress on Medical Physics and Biomedical Engineering, pp. 608-612, 2006.

[204] X. M. Chen, P. U. Mak, S. H. Pun et al., "Study of channel characteristics for galvanic-type Intra-Body communication based on a transfer function from a quasi-static field model," Sensors, vol. 12, no. 12, pp. 16433-16450, 2012.

[205] M. Shinagawa, K. Ochiai, H. Sakamoto, and T. Asahi, "Human area networking technology: RedTacton," NTT Technical Review, vol. 3, no. 5, pp. 41-46, 2005.

[206] A. M. Franz, T. Haidegger, W. Birkfellner, K. Cleary, T. M. Peters, and L. Maier-Hein, "Electromagnetic tracking in medicine A review of technology, validation, and applications," IEEE Transactions on Medical Imaging, vol. 33, no. 8, pp. 1702-1725, 2014.

[207] A. S. Cacciapuoti and M. Caleffi, "Receiver design for a bionic nervous system: Modeling the dendritic processing power," IEEE Internet of Things Journal, vol. 3, no. 1, pp. 27-37, 2016.

[208] I. F. Akyildiz, J. M. Jornet, and M. Pierobon, "Nanonetworks: A new frontier in communications," Communications of the ACM, vol. 54, no. 11, pp. 84-89, 2011.

[209] H. Elayan, R. M. Shubair, J. M. Jornet, and P. Johari, "Terahertz Channel Model and Link Budget Analysis for Intrabody Nanoscale Communication," IEEE Transactions on NanoBioscience, vol. 16, no. 6, pp. 491-503, 2017.

[210] T. Denison, M. Morris, and F. Sun, "Building a bionic nervous system," IEEE Spectrum, vol. 52, no. 2, pp. 32-39, 2015.

[211] F. Mesiti and I. Balasingham, "Nanomachine-to-neuron communication interfaces for neuronal stimulation at nanoscale," IEEE Journal on Selected Areas in Communications, vol. 31, no. 12, pp. 695-704, 2013.

[212] M. Veletić, P. A. Floor, and I. Balasingham, "From nano-scale neural excitability to long term synaptic modification," in Proceedings of the 1st ACM International Conference on Nanoscale Computing and Communication, NANOCOM 2014, USA, May 2014.

[213] J. Suzuki, S. Balasubramaniam, S. Pautot, V. D. Perez Meza, and Y. Koucheryavy, "A Service-Oriented Architecture for Body
Area NanoNetworks with Neuron-based Molecular Communication," Mobile Networks and Applications, vol. 19, no. 6, pp. 707717,2014

[214] L. Galluccio, G. Morabito, and S. Palazzo, "Exploiting timing channel in intra-body sensor networks," in Proceedings of the 2012 IEEE Global Communications Conference, GLOBECOM 2012, pp. 5368-5373, USA, December 2012.

[215] V. De Santis and M. Feliziani, "Intra-body channel characterization of medical implant devices," in Proceedings of the 10th International Symposium on Electromagnetic Compatibility, EMC Europe 2011, pp. 816-819, gbr, September 2011.

[216] X. M. Chen, P. U. Mak, S. H. Pun, Y. M. Gao, M. I. Vai, and M. Du, "Signal transmission through human muscle for implantable medical devices using galvanic intra-body communication technique," in Proceedings of the 34th Annual International Conference of the IEEE Engineering in Medicine and Biology Society, EMBS 2012, pp. 1651-1654, USA, September 2012.

[217] G. Enrico Santagati and T. Melodia, "Experimental Evaluation of Impulsive Ultrasonic Intra-Body Communications for Implantable Biomedical Devices," IEEE Transactions on Mobile Computing, vol. 16, no. 2, pp. 367-380, 2017.

[218] S. Pun, Y. Gao, P. U. Mak et al., "Galvanic intrabody communication for affective acquiring and computing," IEEE Transactions on Affective Computing, vol. 3, no. 2, pp. 145-151, 2012.

[219] E. Okamoto, S. Kikuchi, and Y. Mitamura, "Electrical characteristic of the titanium mesh electrode for transcutaneous intrabody communication to monitor implantable artificial organs," The International Journal of Artificial Organs, vol. 19, no. 3, pp. 257-261, 2016.

[220] M. Sun, M. Mickle, W. Liang, Q. Liu, and R. J. Sclabassi, "Data communication between brain implants and computer," IEEE Transactions on Neural Systems and Rehabilitation Engineering, vol. 11, no. 2, pp. 189-192, 2003.

[221] W. J. Tomlinson, K. R. Chowdhury, and C. Yu, "A multicast communication scheme using weak electrical current for intra-body networks," in Proceedings of the 9th International Conference on Body Area Networks, BODYNETS 2014, pp. 300304, UK, October 2014.

[222] A. S. Cacciapuoti, A. Piras, and M. Caleffi, "Modeling the dynamic processing of the presynaptic terminals for intrabody nanonetworks," IEEE Transactions on Communications, vol. 64, no. 4, pp. 1636-1645, 2016.

[223] Y. Chahibi and I. Balasingham, "An intra-body molecular communication networks framework for continuous health monitoring and diagnosis," in Proceedings of the 37th Annual International Conference of the IEEE Engineering in Medicine and Biology Society, EMBC 2015, pp. 4077-4080, Italy, August 2015.

[224] I. F. Akyildiz, M. Pierobon, S. Balasubramaniam, and Y. Koucheryavy, "The internet of Bio-Nano things," IEEE Communications Magazine, vol. 53, no. 3, pp. 32-40, 2015.

[225] S. J. Lee, C. (Andrew) Jung, K. Choi, and S. Kim, "Design of wireless nanosensor networks for intrabody application," International Journal of Distributed Sensor Networks, vol. 2015, Article ID 176761, 12 pages, 2015.

[226] D. Malak and O. B. Akan, "Communication theoretical understanding of intra-body nervous nanonetworks," IEEE Communications Magazine, vol. 52, no. 4, pp. 129-135, 2014. 
[227] M. Pierobon, "A molecular communication system model based on biological circuits," in Proceedings of the 1st ACM International Conference on Nanoscale Computing and Communication, NANOCOM 2014, USA, May 2014.

[228] I. F. Akyildiz and J. M. Jornet, "Electromagnetic wireless nanosensor networks," Nano Communication Networks, vol. 1, no. 1, pp. 3-19, 2010.

[229] D. Malak and O. B. Akan, "A communication theoretical analysis of synaptic multiple-access channel in hippocampal-cortical neurons," IEEE Transactions on Communications, vol. 61, no. 6, pp. 2457-2467, 2013.

[230] K. Yang, A. Pellegrini, M. O. Munoz, A. Brizzi, A. Alomainy, and Y. Hao, "Numerical analysis and characterization of $\mathrm{THz}$ propagation channel for body-centric nano-communications," IEEE Transactions on Terahertz Science and Technology, vol. 5, no. 3, pp. 419-426, 2015.

[231] H. Zhu, R. Xu, and J. Yuan, "High speed intra-body communication for personal health care," in Proceedings of the Annual International Conference of the IEEE Engineering in Medicine and Biology Society (EMBC '09), pp. 709-712, IEEE, Minneapolis, Minn, USA, September 2009. 


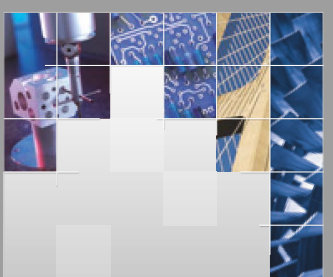

\section{Enfincering}
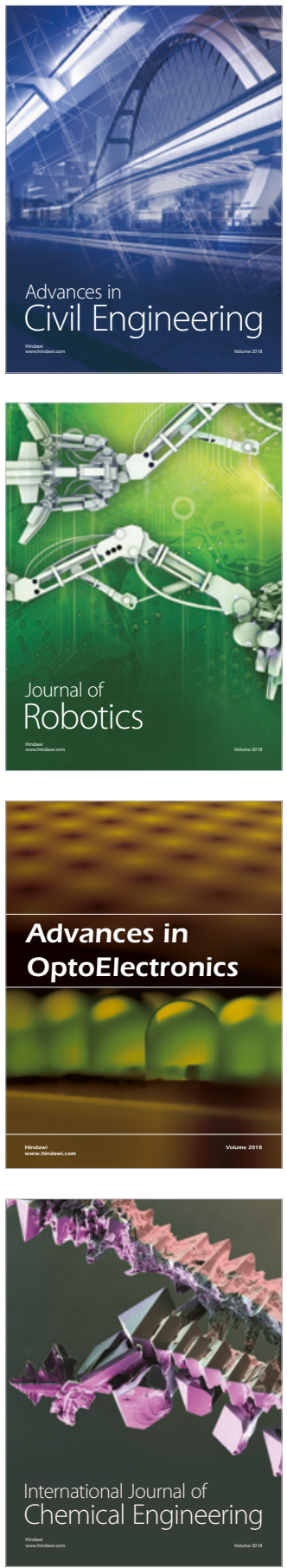

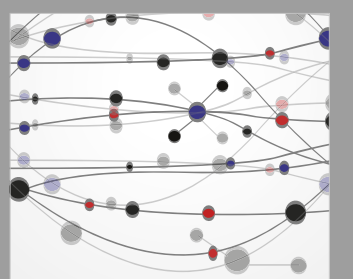

\section{Rotating \\ Machinery}

The Scientific World Journal

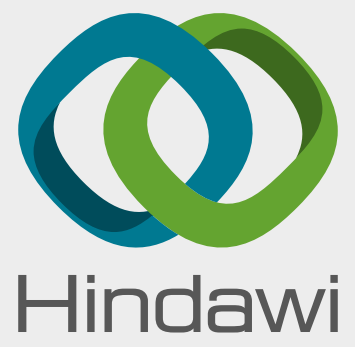

Submit your manuscripts at

www.hindawi.com
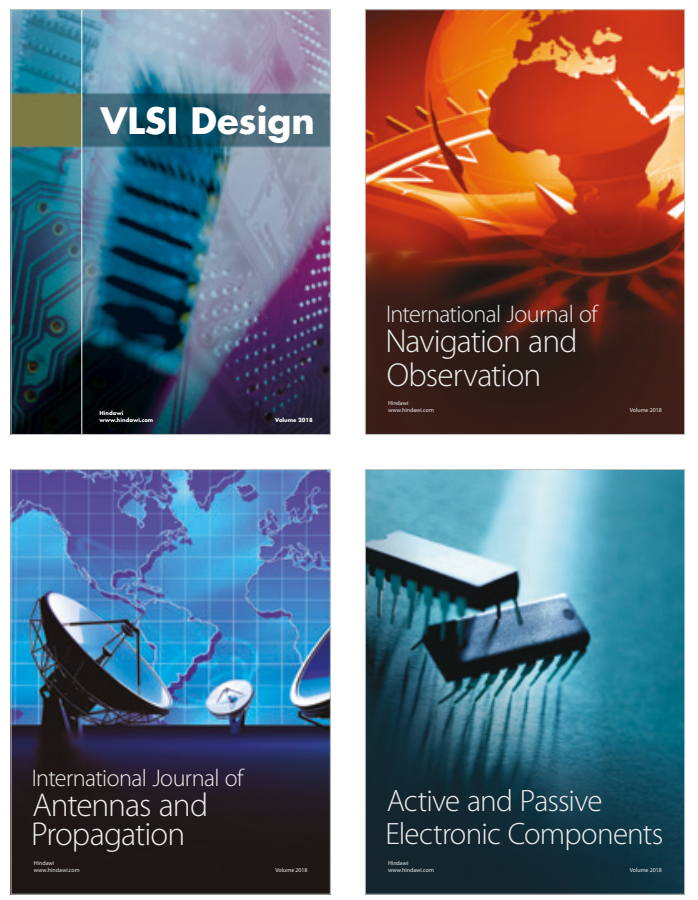
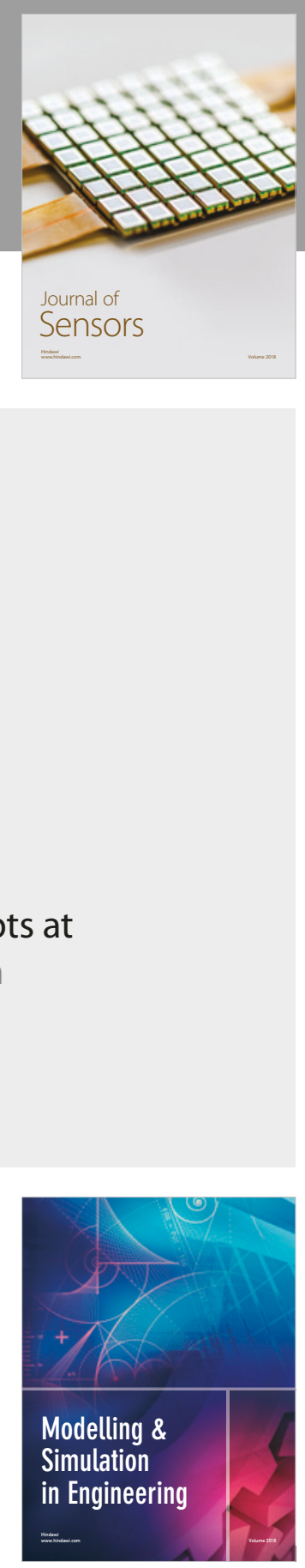

\section{Advances \\ Multimedia}
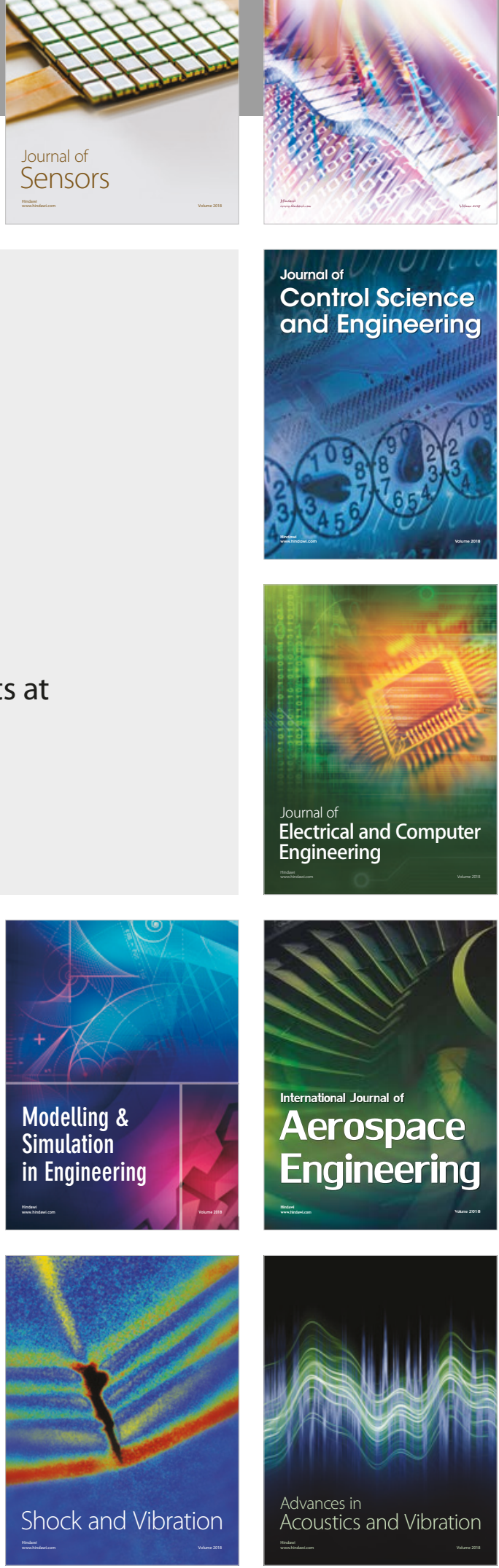